\title{
Quantum mechanical reactive scattering for planar atom plus diatom systems. I. Theory*
}

\author{
Aron Kuppermann, George C. Schatz, ${ }^{\dagger}$ and M. Baer ${ }^{\ddagger}$ \\ Arthur Amos Noyes Laboratory of Chemical Physics, ${ }^{\S}$ California Institute of Technology, Pasadena, \\ California 91125 \\ (Received 22 December 1975)
}

\begin{abstract}
A method is presented for accurately solving the Schrödinger equation for the reactive collision of an atom with a diatomic molecule on a space-fixed plane. The procedure consists primarily of two steps. First, the Schrödinger equation in each of the three arrangement channel regions is transformed into a set of coupled differential equations and numerically integrated in each of these regions to generate primitive solutions. The rotational part of the vibration-rotation basis functions involved is not changed from its asymptotic form during this propagation, but the vibrational eigenfunctions as well as the integration variable are changed periodically so as to follow the vibrational motions in a nearly adiabatic manner. In the second step, the primitive solutions generated in each of the three arrangement channels are smoothly matched to each other on a set of appropriately chosen matching surfaces. The resulting solutions are then linearly combined to satisfy the proper asymptotic boundary conditions, and the scattering matrix, scattering amplitudes, and cross sections are determined. Application of this procedure to the special case of the $\mathbf{H}+\mathrm{H}_{2}$ reaction is discussed in detail including simplifications arising from the additional symmetries involved, and the inclusion of effects resulting from indistinguishability of identical particles.
\end{abstract}

\section{INTRODUCTION}

In recent years, much interest has developed in the $a b$ initio calculation of bimolecular reaction cross sections on realistic potential energy surfaces by accurate quantum mechanical techniques. The motivation for such calculations has been to interpret the results of crossed molecular beam experiments, to understand the effect of the relative translational energy of the reactants and of their internal state on such cross sections and on the disposal of energy among the reaction products, to elucidate the role of direct and compound state dynamical mechanisms, to test the range and degree of validity of approximate reaction models (such as the adiabatic and statistical ones), to develop new physical models of known reliability, to examine the correctness of the dynamical assumptions of transition state theory, to establish the conditions of applicability of the quasiclassical trajectory calculations and of semiclassical improvements thereof, and last, but not least, to make detailed qualitative and quantitative predictions from first principles about reactions difficult to investigate experimentally.

Owing in part to the lack of appropriate numerical techniques and to limitations of the memory size and computational speed and cost of present-day large computers, most of these quantum calculations so far have been performed for collinear atom-diatom reactions ${ }_{0}{ }^{1-13}$ In recent years, several attempts have been made to do calculations for nonlinear triatomic systems. Saxon and Light ${ }^{14}$ and Altenberger-Siczek and Light ${ }^{15}$ have investigated the coplanar $\mathrm{H}+\mathrm{H}_{2}$ exchange reaction by a coupled-channel technique which excluded closed vibrational channels. Wolken and Karplus ${ }^{16}$ have made a study of this same reaction in three dimensions, also ignoring closed vibrational channels. Wyatt and coworkers ${ }^{17}$ have developed techniques which include closed vibrations in calculations on one and two reaction path atom plus diatom reactions, and Elkowitz and Wyatt $^{17 a}$ have applied these methods to the three-dimensional $\mathrm{H}+\mathrm{H}_{2}$ reaction. Baer and $\mathrm{Kouri}^{18}$ have done fully converged calculations in three dimensions on a model (one reaction path) triatomic system in which one of the atoms was assumed infinitely heavy and in which the simple potential used allowed a partially analytic treatment.

In an earlier communication, ${ }^{19}$ we presented preliminary results of the first fully converged quantum mechanical calculation for a coplanar reaction on a realistic electronically adiabatic potential energy surface, that for $\mathrm{H}+\mathrm{H}_{2}$. These results indicated that the quantitative differences between calculations in which closed vibrations are included and those for which they are not can be quite serious. In this paper, we will present a detailed description of the method used to perform such calculations. We will formulate the method for a general atom plus diatomic molecule collision on a single reactive potential energy surface using $\mathrm{H}+\mathrm{H}_{2}$ as a specific example. A detailed description of the results of our extensive calculations on coplanar $\mathrm{H}+\mathrm{H}_{2}$ will be given elsewhere. ${ }^{20}$

The method is based on an earlier coupled-channel (i.e., close-coupling) propagation technique ${ }^{5}$ which has been extensively applied to the collinear $\mathrm{H}+\mathrm{H}_{2}$ and $\mathrm{F}$ $+\mathrm{H}_{2}$ reactions ${ }^{10}$ The spirit of the collinear method is to choose different variables and different pseudovibrational basis functions for expansion of the solutions of the Schrödinger equation in different local regions of the (collinear) configuration space so as to minimize the number of terms needed for accurate convergence of the expansions. This concept is retained for the vibrational motion in our application of the method to coplanar collisions. However, the variables and basis functions describing rotational motion are not changed (and hence retain their asymptotic meanings) during the integration into the interaction region from each of the three separated arrangement channel regions of internal configuration space. As a result, an additional step is required at the completion of the integration in which the primitis solutions in each of the three arrangement channel reqions are smoothly matched to each other on a set of 
three appropriately chosen surfaces which separate these three regions. ${ }^{21}$ The restriction that the three atoms should be confined to a space-fixed plane was introduced for computational convenience to test out the effectiveness of the method without excessive expenditure of compution time. Extension to reactions in three. dimensional space is reasonably straightforward and has recently been implemented by Kuppermann and Schatz ${ }^{22}$ for $3 \mathrm{D} \mathrm{H}+\mathrm{H}_{2}$ in the first fully converged quantum mechanical treatment of a chemical reaction on a realistic potential energy surface.

In Sec. II of this paper we formulate the Schrödinger equation for the problem and describe the partial wave expansion used to obtain the partial differential equations, in internal configuration coordinates, which must be solved. The method used to integrate these equations in the different regions of configuration space is described in Sec. III, and in Sec. IV we describe how we smoothly match the solutions obtained from these integrations. The asymptotic analysis is developed in Sec. V, including the methods of calculating the reactance and scattering matrices, the scattering amplitudes, and the cross sections. Finally, in Sec. VI we describe the simplifications and changes involved in an application of the method to the $\mathrm{H}+\mathrm{H}_{2}$ exchange reaction due to the identicity of the three atoms, including a discussion of the technique of antisymmetrizing the scattering wavefunction (post antisymmetrization) in a way which is applicable to the three-dimensional case also.

\section{FORMULATION OF THE PROBLEM}

\section{A. General considerations}

We are interested in calculating cross sections for the exchange reaction $\mathrm{A}+\mathrm{BC}-\mathrm{AB}+\mathrm{C}, \mathrm{AC}+\mathrm{B}$ in which the three atoms $A, B$, and $C$ are confined to remain on a space-fixed plane. We assume that the Born-Oppenheimer separation approximation between the electron and nuclear motions is valid and that the resulting ground electronic state potential energy surface $V$ is known. We further assume that all higher potential energy surfaces are sufficiently greater than the total energy $E$ of the system everywhere in configuration space for us to be able to neglect their influence on the cross sections. This "single potential energy surface" model is applicable to a high degree of accuracy to many triatomic reactions. We also assume that $E$ is sufficiently low for the existence and effect of break-up collisions of the type $\mathrm{A}+\mathrm{BC}-\mathrm{A}+\mathrm{B}+\mathrm{C}$ to be negligible. Finally, we assume that the interactions between the nuclear spin and nuclear orbital angular momenta are negligible.

Let $r_{A}, r_{B}$, and $r_{C}$ be the position vectors of nuclei $A, B$, and $C$, respectively, with respect to their center of mass. We wish to obtain a solution to the time-independent Schrödinger equation for the motion of the nuclei which satisfies the following three conditions:

(a) In configuration space $r_{A}, r_{B}, r_{C}$ it is everywhere single valued and continuous and has gradients which are everywhere continuous (except at points in that space for which two of the three atoms coincide).

(b) It is antisymmetric (symmetric) with respect to exchange of the space and spin coordinates of any two identical nuclei of half odd-integral (integral) nuclear spin.

(c) It satisfies the asymptotic conditions describing the collision phenomenon under consideration, $i_{0} e_{0}$, the collision of $\mathrm{A}$ and $\mathrm{BC}$ with a given relative kinetic energy and a given initial internal quantum state of $B C$, to produce receding products $\mathrm{A}+\mathrm{BC}, \mathrm{AB}+\mathrm{C}$, or $\mathrm{AC}+\mathrm{B}$ in all possible internal states of the diatom compatible with the total energy of the system.

If we can find a sufficient number of independent solutions satisfying Condition (a), it is possible to obtain linear combinations of them which, in addition, satisfy Conditions (b) (by postantisymmetrization methods ${ }^{23}$ ) and $(c)$ (by reactance or scattering matrix analysis techniques ${ }^{24}$ ).

\section{B. The Schrödinger equation}

Let $\overline{\mathbf{R}}_{\alpha}$ be the position vector of $\mathrm{A}$. with respect to the center of mass of $\mathrm{BC}$ and $\overline{\mathrm{r}}_{\alpha}$ the position vector of $\mathrm{C}$ with respect to $\mathrm{B}$, as indicated in Fig。 1 . As $\bar{R}_{\alpha} \rightarrow \infty$ with $\bar{\gamma}_{\alpha}$ remaining finite, we denote the corresponding configuration as arrangement channel $\alpha \equiv(\mathrm{A}, \mathrm{BC})$. Let $\left(\bar{r}_{\alpha}, \theta_{r_{\alpha}}\right)$ and $\left(\bar{R}_{\alpha}, \theta_{\alpha}\right)$ be, respectively, the planar polar coordinates of $\overline{\mathbf{r}}_{\alpha}$ and $\overline{\mathbf{R}}_{\alpha}$ with respect to a fixed system of reference attached to the plane (all angles being measured from the $O x$ axis of Fig. 1). The reduced mass associated to the motion of $C$ with respect to $B$ is denoted by $\mu_{\beta \gamma}$ and that associated to atom $A$ with respect to molecule $\mathrm{BC}$ is $\mu_{\alpha, \beta \gamma}$. We define analogous vectors $\overline{\mathbf{r}}_{\beta}, \overline{\mathbf{R}}_{\beta}$ and $\overline{\mathbf{r}}_{\gamma}, \overline{\mathbf{R}}_{\gamma}\left(\mathrm{Fig}_{\circ} 1\right.$ ) and associated coordinates and reduced masses so that as $\bar{R}_{\beta} \rightarrow \infty$ or $\bar{R}_{\gamma} \rightarrow \infty$ with $\bar{r}_{\beta}$ or $\bar{r}_{\gamma}$ finite, we obtain the arrangement channels $\beta$ $\equiv(\mathrm{B}, \mathrm{CA})$ and $\gamma \equiv(\mathrm{C}, \mathrm{AB})$, respectively. Note that the directions of the vectors in Fig. 1 are defined in a cyclic manner in the indices $\alpha \beta \gamma$. Finally, let $\lambda \nu \kappa$ represent any one of the three possible cyclic permutations $\alpha \beta \gamma, \beta \gamma \alpha$, and $\gamma \alpha \beta$ 。

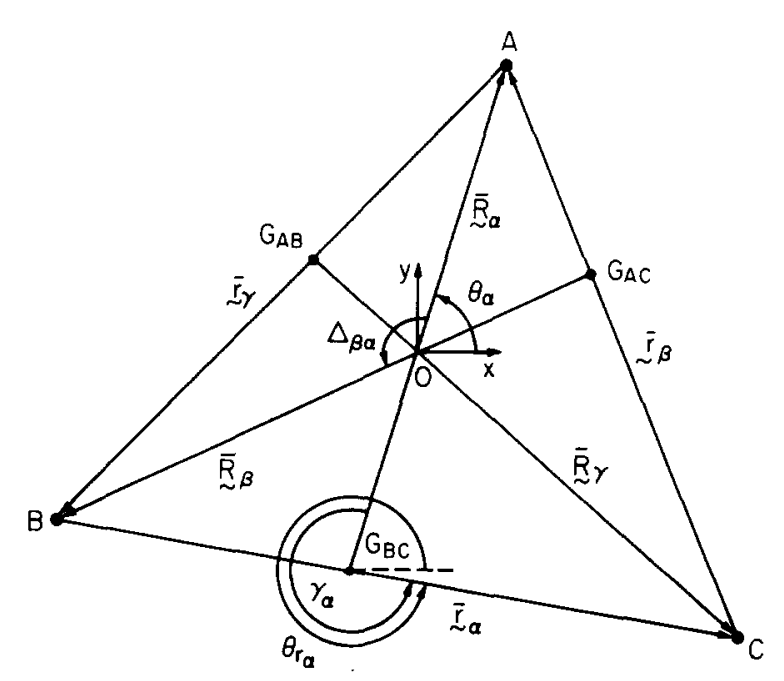

FIG. 1. Vectors used to specify the location of three atoms in the $\mathrm{A}, \mathrm{B}, \mathrm{C}$ collision system relative to the center of mass $O$. $G_{\mathrm{BC}}, G_{\mathrm{AC}}$, and $G_{\mathrm{AB}}$ denote the locations of the centers of mass of the diatomic, $\mathrm{BC}, \mathrm{AC}$, and $\mathrm{AB}$, respectively. $\overrightarrow{\mathrm{R}}_{\alpha}, \mathbf{F}_{\alpha}, \overline{\mathrm{R}}_{\beta}$, $\overline{\mathbf{r}}_{\beta}, \overline{\mathrm{R}}_{\gamma}, \overline{\mathbf{r}}_{\gamma}$ are defined in text. 
In the system of coordinates characterized by index $\lambda(=\alpha, \beta$ or $\gamma)$, the Schrödinger equation describing the internal motion of the three-particle system is

$$
\begin{aligned}
& {\left[-\frac{\hbar^{2}}{2 \mu_{\nu \kappa}}\left(\frac{1}{\bar{r}_{\lambda}} \frac{\partial}{\partial \bar{r}_{\lambda}} \bar{r}_{\lambda} \frac{\partial}{\partial \bar{r}_{\lambda}}+\frac{1}{\bar{r}_{\lambda}^{2}} \frac{\partial^{2}}{\partial \theta_{r_{\lambda}}^{2}}\right)\right.} \\
& -\frac{\hbar^{2}}{2 \mu_{\lambda, \nu \kappa}}\left(\frac{1}{\bar{R}_{\lambda}} \frac{\partial}{\partial \bar{R}_{\lambda}} \bar{R}_{\lambda} \frac{\partial}{\partial \bar{R}_{\lambda}}+\frac{1}{\bar{R}_{\lambda}^{2}} \frac{\partial^{2}}{\partial \theta_{\lambda}^{2}}\right) \\
& \left.\quad+V^{\lambda}\left(\bar{r}_{\lambda}, \bar{R}_{\lambda}, \gamma_{\lambda}\right)-E\right] \Psi^{\lambda}\left(\bar{r}_{\lambda}, \bar{R}_{\lambda}, \theta_{r_{\lambda}}, \theta_{\lambda}\right)=0,
\end{aligned}
$$

where $V^{\lambda}$ is the potential energy function of the system expressed in $\lambda$ coordinates, and $E$ is its total energy。 $\gamma_{\lambda}$ is by definition the counterclockwise angle from $\bar{R}_{\lambda}$ to $\bar{r}_{\lambda^{\circ}}$ It lies in the 0 to $2 \pi$ range and is equal to $\theta_{r_{\lambda}}-\theta_{\lambda}$ modulo $2 \pi$. In the absence of external fields, the potential function depends only on the internal variables $\bar{R}_{\lambda}, \bar{r}_{\lambda}, \gamma_{\lambda}$ and satisfies the relation $V^{\lambda}\left(\bar{R}_{\lambda}, \bar{r}_{\lambda}, \gamma_{\lambda}\right)=V^{\lambda}\left(\bar{R}_{\lambda}\right.$, $\left.\bar{\gamma}_{\lambda}, 2 \pi-\gamma_{\lambda}\right)$.

Let us now introduce a set of coordinates used previously by Delves, ${ }^{25} \mathrm{Jepsen}$ and Hirschfelder, ${ }^{26}$ and Smith. ${ }^{27}$ They have the advantage of leading to an equation containing a single effective mass $\mu$ which is independent of the arrangement channel $\lambda$; this simplifies many of the equations presented below. These coordinates are

$$
\begin{aligned}
& \mathbf{r}_{\lambda}=a_{\lambda}^{-1} \overline{\mathbf{r}}_{\lambda}, \\
& \mathbf{R}_{\lambda}=a_{\lambda} \overline{\mathbf{R}}_{\lambda},
\end{aligned}
$$

where $a_{\lambda}$ is a dimensionless scaling constant defined by

$$
a_{\lambda}=\left(\mu_{\lambda, \nu k} / \mu_{\nu k}\right)^{1 / 4} \text {. }
$$

Substitution into $\mathrm{Eq}$. $(2.1)$ leads to

$$
\begin{gathered}
{\left[-\frac{\hbar^{2}}{2 \mu}\left(\frac{1}{r_{\lambda}} \frac{\partial}{\partial r_{\lambda}} r_{\lambda} \frac{\partial}{\partial r_{\lambda}}+\frac{1}{r_{\lambda}^{2}} \frac{\partial^{2}}{\partial \theta_{r_{\lambda}}^{2}}+\frac{1}{R_{\lambda}} \frac{\partial}{\partial R_{\lambda}} R_{\lambda} \frac{\partial}{\partial R_{\lambda}}+\frac{1}{R_{\lambda}^{2}} \frac{\partial^{2}}{\partial \theta_{\lambda}^{2}}\right)\right.} \\
\left.+V^{\lambda}\left(r_{\lambda}, R_{\lambda}, r_{\lambda}\right)-E\right] \Psi^{\lambda}\left(r_{\lambda}, R_{\lambda}, \theta_{r_{\lambda}}, \theta_{\lambda}\right)=0,
\end{gathered}
$$

where the circular polar angles of $\mathbf{R}_{\lambda}, \mathbf{r}_{\lambda}$ are the same as those of $\overline{\mathbf{R}}_{\lambda}, \overline{\mathbf{r}}_{\lambda}$, and $\mu$ is the effective mass alluded to above and defined by

$$
\mu=\left(\mu_{\nu k} \mu_{\lambda, \nu k}\right)^{1 / 2}=\left(m_{\alpha} m_{\beta} m_{\gamma} / M\right)^{1 / 2},
$$

where

$$
M=m_{\alpha}+m_{\beta}+m_{\gamma}
$$

is the total mass of the system, and $m_{\alpha}, m_{\beta}$, and $m_{\gamma}$ are the masses of atoms $\mathrm{A}, \mathrm{B}$, and $\mathrm{C}$, respectively. We now change from the angular variables $\theta_{r_{\lambda}}, \theta_{\lambda}$ to $\gamma_{\lambda}, \theta_{\lambda}$, obtaining the following expression from $\mathrm{Eq}$. $(2.4)$ :

$$
\begin{aligned}
& \left\{-\frac{\hbar^{2}}{2 \mu}\left[\frac{1}{r_{\lambda}} \frac{\partial}{\partial r_{\lambda}} r_{\lambda} \frac{\partial}{\partial r_{\lambda}}+\frac{1}{r_{\lambda}^{2}} \frac{\partial^{2}}{\partial \gamma_{\lambda}^{2}}+\frac{1}{R_{\lambda}} \frac{\partial}{\partial R_{\lambda}} R_{\lambda} \frac{\partial}{\partial R_{\lambda}}\right.\right. \\
& \left.+\frac{1}{R_{\lambda}^{2}}\left(\frac{\partial^{2}}{\partial \theta_{\lambda}^{2}}-2 \frac{\partial^{2}}{\partial \theta_{\lambda} \partial \gamma_{\lambda}}+\frac{\partial^{2}}{\partial \gamma_{\lambda}^{2}}\right)\right]+V^{\lambda}\left(r_{\lambda}, R_{\lambda}, \gamma_{\lambda}\right) \\
& \quad-E\} \Psi^{\lambda}\left(r_{\lambda}, R_{\lambda}, \gamma_{\lambda}, \theta_{\lambda}\right)=0 .
\end{aligned}
$$

This is called the body-fixed Schrödinger equation, because $\gamma_{\lambda}$ is the angle of $\boldsymbol{r}_{\lambda}$ with respect to the body-fixed
$\mathbf{R}_{\lambda}$ direction rather than the laboratory-fixed $O x$ axis. Similarly, $R_{\lambda}, \theta_{\lambda}, r_{\lambda}, \gamma_{\lambda}$ are called the body-fixed $\lambda$ coordinates. The independence of $V^{\lambda}$ on $\theta_{\lambda}$ in the above equation introduces very convenient simplifications, as is shown in the next section.

\section{Partial wave expansion}

The total angular momentum $J$ of the triatomic system (with respect to its center of mass) is a constant of the motion which commutes with the Hamiltonian $H$ 。 The solution of the Schrödinger equation we are seeking, satisfying Conditions (a), (b), and (c) of Sec. II.A, is not an eigenfunction of $\mathrm{J}_{\circ}{ }^{28}$ It is, however, convenient to perform the coplanar analog of partial wave analysis by expanding $\Psi^{\lambda}$ in terms of the simultaneous eigenfunctions $\Psi_{J}^{\lambda}$ of $H$ and $\mathrm{J}_{\text {。 }}$

In the system of coordinates $r_{\lambda}, R_{\lambda}, \theta_{r_{\lambda}}, \theta_{\lambda}$ the operator $\mathrm{J}$ is given by

$$
J=\left[\frac{\hbar}{i}\left(\frac{\partial}{\partial \theta_{\lambda}}\right)_{\theta_{r_{\lambda}}}+\frac{\hbar}{i}\left(\frac{\partial}{\partial \theta_{r_{\lambda}}}\right)_{\theta_{\lambda}}\right] \hat{\mathbf{z}} \quad \lambda=\alpha, \beta, \gamma,
$$

where $\hat{\mathbf{z}}$ is the unit vector perpendicular to the fixed plane of motion of the three particles. We recognize from Eq. (2.7) that $J$ is the algebraic sum of the rotational angular momentum $j_{\lambda}=(\hbar / i)\left(\partial / \partial \theta_{r_{\lambda}}\right)_{\theta_{\lambda}} \hat{\mathbf{z}}$ and the orbital angular momentum $1_{\lambda}=(\hbar / i)\left(\partial / \partial \theta_{\lambda}\right)_{\theta_{r}} \hat{\mathbf{z}}$. Transforming $\mathrm{J}$ to the angular variables $\theta_{\lambda}, \gamma_{\lambda}$, we get

$$
J=\frac{\hbar}{i}\left(\frac{\partial}{\partial \theta_{\lambda}}\right)_{\gamma_{\lambda}} \hat{\mathbf{z}} \quad \lambda=\alpha, \beta, \gamma,
$$

which may be physically interpreted as indicating that if $\gamma_{\lambda}$ is maintained constant, a variation of the angular coordinate $\theta_{\lambda}$ of $\mathbf{R}_{\lambda}$ produces a rotation of the entire triatomic system.

The orthonormal eigenfunctions of $\mathrm{J}$ are given by ${ }^{29}$

$$
\varphi_{J}\left(\theta_{\lambda}\right)=(2 \pi)^{-1 / 2} \exp \left(i J \theta_{\lambda}\right) \quad J=0, \pm 1, \pm 2, \ldots
$$

The simultaneous eigenfunctions of $H$ with energy $E$ and of $\mathbf{J}$ with total angular momentum $J \hbar$ are of the form

$$
\Psi_{J}^{\lambda}\left(r_{\lambda}, R_{\lambda}, \gamma_{\lambda}, \theta_{\lambda}\right)=\varphi_{J}\left(\theta_{\lambda}\right) \chi_{J}^{\lambda}\left(r_{\lambda}, R_{\lambda}, \gamma_{\lambda}\right),
$$

where

$$
\begin{aligned}
\left\{-\frac{\hbar^{2}}{2 \mu}\right. & {\left[\frac{1}{r_{\lambda}} \frac{\partial}{\partial r_{\lambda}} r_{\lambda} \frac{\partial}{\partial r_{\lambda}}+\frac{1}{r_{\lambda}^{2}} \frac{\partial^{2}}{\partial \gamma_{\lambda}^{2}}+\frac{1}{R_{\lambda}} \frac{\partial}{\partial R_{\lambda}} R_{\lambda} \frac{\partial}{\partial R_{\lambda}}\right.} \\
& \left.+\frac{1}{R_{\lambda}^{2}}\left(-J^{2}-2 i J \frac{\partial}{\partial \gamma_{\lambda}}+\frac{\partial^{2}}{\partial \gamma_{\lambda}^{2}}\right)\right]+V^{\lambda}\left(r_{\lambda}, R_{\lambda}, \gamma_{\lambda}\right) \\
& -E\} \chi_{J}^{\lambda}\left(r_{\lambda}, R_{\lambda}, \gamma_{\lambda}\right)=0 .
\end{aligned}
$$

Any solution $\Psi^{\lambda}$ of the Schrödinger equation which is not necessarily an eigenfunction of $\mathbf{J}$ can be written as

$$
\Psi^{\lambda}\left(r_{\lambda}, R_{\lambda}, \gamma_{\lambda}, \theta_{\lambda}\right)=\sum_{J=-\infty}^{\infty} C_{J}^{\lambda} \Psi_{J}^{\lambda}\left(r_{\lambda}, R_{\lambda}, \gamma_{\lambda}, \theta_{\lambda}\right) 。
$$

The constant coefficients $C_{J}^{\lambda}$ appropriate for the problem being considered will be determined by the asymptotic conditions specified in Requirement (c) of Sec. $\Pi_{.} \mathrm{A}$ and will be discussed in Sec. V.B.

We now simplify Eq. (2.11) by defining a new function $\psi_{J}^{\lambda}$ by 


$$
\psi_{J}^{\lambda}\left(r_{\lambda}, R_{\lambda}, \gamma_{\lambda}\right)=\left(r_{\lambda} R_{\lambda}\right)^{1 / 2} \chi_{J}^{\lambda}\left(r_{\lambda}, R_{\lambda}, \gamma_{\lambda}\right) \text {. }
$$

Substituting this into Eq. $(2,11)$, we obtain

$$
\begin{gathered}
\left\{-\frac{\hbar^{2}}{2 \mu}\left[\frac{\partial^{2}}{\partial r_{\lambda}^{2}}+\frac{\partial^{2}}{\partial R_{\lambda}^{2}}+\frac{1}{r_{\lambda}^{2}} \frac{\partial^{2}}{\partial \gamma_{\lambda}^{2}}+\frac{1}{R_{\lambda}^{2}}\left(-J^{2}-2 i J \frac{\partial}{\partial \gamma_{\lambda}}+\frac{\partial^{2}}{\partial \gamma_{\lambda}^{2}}\right)\right]\right. \\
\left.+V^{\lambda}-E\right\} \psi_{J}^{\lambda}\left(r_{\lambda}, R_{\lambda}, \gamma_{\lambda}\right)=0 .
\end{gathered}
$$

This equation is solved numerically by the method described in Secs. III and IV.

\section{INTEGRATION OF THE SCHRÖDINGER EQUATION}

\section{A. The partitioning of configuration space into arrangement channel regions}

We now wish to expand $\psi_{J}^{\lambda}\left(r_{\lambda}, R_{\lambda}, \gamma_{\lambda}\right)$ in terms of sets of two-variable internal state basis functions in order to reduce the partial differential equation $(2.14)$ to a system of coupled ordinary differential equations. Our choice for the internal state basis functions and the corresponding variables will be different in different regions of the three-dimensional internal configuration space $r_{\lambda}, R_{\lambda}, \gamma_{\lambda}$ and will be largely determined by the local shape of the potential energy function $V^{\lambda}\left(r_{\lambda}, R_{\lambda}, \gamma_{\lambda}\right)$. This is done in order to represent solutions of the full Schrödinger equation in an efficient manner in all regions of configuration space so as to reduce computation time as much as possible. A useful conceptualization of the nature of the problem can be gained by noting the appearance of the potential energy function $V^{\lambda}\left(r_{\lambda}\right.$, $\left.R_{\lambda}, \gamma_{\lambda}\right)$. This function is most conveniently displayed with the aid of a mapping procedure previously developed for this purpose. ${ }^{30}$ In this mapping we consider a space $O X_{\lambda} Y_{\lambda} Z_{\lambda}$ in which a point $P$ has spherical polar coordinates $\zeta, \omega_{\lambda}, \gamma_{\lambda}$, where the radial variable $\zeta$ is defined by

$$
\zeta=\left(r_{\lambda}^{2}+R_{\lambda}^{2}\right)^{1 / 2}
$$

and is independent of $\lambda$ as shown in $E q_{0}$ (A6) of Appen$\operatorname{dix} A$, and the polar angle $\omega_{\lambda}$ is defined by

$$
\omega_{\lambda}=2 \tan ^{-1}\left(r_{\lambda} / R_{\lambda}\right), \quad 0 \leq \omega_{\lambda} \leq \pi \text { 。 }
$$

The azimuthal angle $\gamma_{\lambda}$ has been defined after Eq. (2.1). Using the example of the $\mathrm{H}+\mathrm{H}_{2}$ reaction, the resulting contour plot of the Porter-Karplus ${ }^{31}$ potential for six different values of $\gamma_{\lambda}$ is depicted in Fig. 2. This representation has the advantage of treating all three arrangement channels equivalently in that a change from coordinates $\lambda$ to coordinates $\nu$ produces a clockwise rotation without distortion of Fig. 2 around the $O Y_{\lambda}$ axis by an angle of $120^{\circ} .^{30}$ We see from the figure that the accessible areas of configuration space are in the form of three tubelike regions whose mutual intersection defines the three-particle interaction region. For less symmetric potentials Fig. 2 would be less symmetric, but the considerations below have general validity.

Our approach for solving the Schrödinger equation consists of dividing the configuration space depicted in Fig. 2 into three subspaces called arrangement channel regions and labeled by the indices $\lambda, \nu, \kappa$. Each of these three arrangement channel regions includes one of the three tubes corresponding to a separated atom plus diatom, along with that part of the interaction region which retains the "general appearance" of that particular tube. For the $\mathrm{H}+\mathrm{H}_{2}$ system, a very natural separation of the three arrangement channel regions is obtained by the use of the three half-planes $\pi_{\nu \lambda}, \pi_{k \nu}$, and $\pi_{\lambda \kappa}$ whose common edge is the $O Y_{\lambda}$ axis and which intersect the $O X_{\lambda} Z_{\lambda}$ plane in the symmetrical positions depicted in Fig。2(a). A general definition of these three half-planes which is also applicable to reactions other than $\mathrm{H}+\mathrm{H}_{2}$ is

$$
\begin{array}{lll}
\pi_{\nu \lambda}: & r_{\lambda}=r_{\nu} & 0 \leqslant \gamma_{\lambda} \leqslant \pi / 2 \text { and } 3 \pi / 2 \leqslant \gamma_{\lambda}<2 \pi, \\
\pi_{\kappa \nu}: & r_{\nu}=r_{\kappa} & 0 \leqslant \gamma_{\nu} \leqslant \pi / 2 \text { and } 3 \pi / 2 \leqslant \gamma_{\nu}<2 \pi, \\
\pi_{\lambda \kappa}: & r_{\kappa}=r_{\lambda} & 0 \leqslant \gamma_{\kappa} \leqslant \pi / 2 \text { and } 3 \pi / 2 \leqslant \gamma_{\kappa}<2 \pi .
\end{array}
$$

A proof that these equations do indeed define half-planes whose edge is the $O Y_{\lambda}$ axis, as graphically indicated in Fig. 2(a), is given in Appendix A along with the equations describing the $\lambda$ to $\nu$ coordinate transformation. For some reactions, the half-planes defined above may be inadequate as they may not separate the three arrangement channel regions into physically intuitive ones as determined by the shape of the potential functions. In these cases, alternative surfaces may be chosen and treated by a straightforward extension of the theory presented in this paper. For the reactions $\mathrm{H}+\mathrm{H}_{2}, \mathrm{D}+\mathrm{H}_{2}$, $H+D_{2}$, etc. , the half-planes of Eqs。 $(3,1)$ are quite satisfactory, so we shall proceed to formulate our theory using the arrangement channel regions into which they divide internal configuration space.

With the arrangement channel regions thus defined, we organize the work involved in solving $\mathbf{E q} .(2,14)$ into two steps. First, within each of the three arrangement channel regions, we integrate the Schrödinger equation using rotational coordinates and basis functions appropriate to the asymptotic part of that arrangement channel but changing vibrational coordinates and basis functions in a way which transforms "smoothly" from one arrangement channel to another. Then, we match the resulting three sets of solutions to one another on the three half-planes $\pi_{\nu \lambda}, \pi_{k \nu}, \pi_{\lambda k}$ described above (and hereafter called the matching surfaces). Since the vibrational coordinates are designed to transform smoothly from one arrangement channel to the others, it is primarily the rotational parts of the wavefunctions which must be considered in the matching procedure. Our rotational expansion is similar to that of Saxon and Light, ${ }^{14}$ but not to that of Wyatt and co-workers, ${ }^{17}$ who allow both their vibrational and rotational coordinates to transform smoothly in going from one arrangement channel to the next.

In the remainder of this section, we will detail the method used for integrating the Schrödinger equation in each of the arrangement channel regions. The crucial smooth matching procedure is outlined in Sec. IV.

\section{B. The rotationally coupled Schrödinger equations}

We now consider the solution of $\mathrm{Eq}_{0}(2.14)$ in each of the three arrangement channel regions $\lambda=\alpha, \beta, \gamma_{0}$

The body-fixed rotational angular momentum operator 

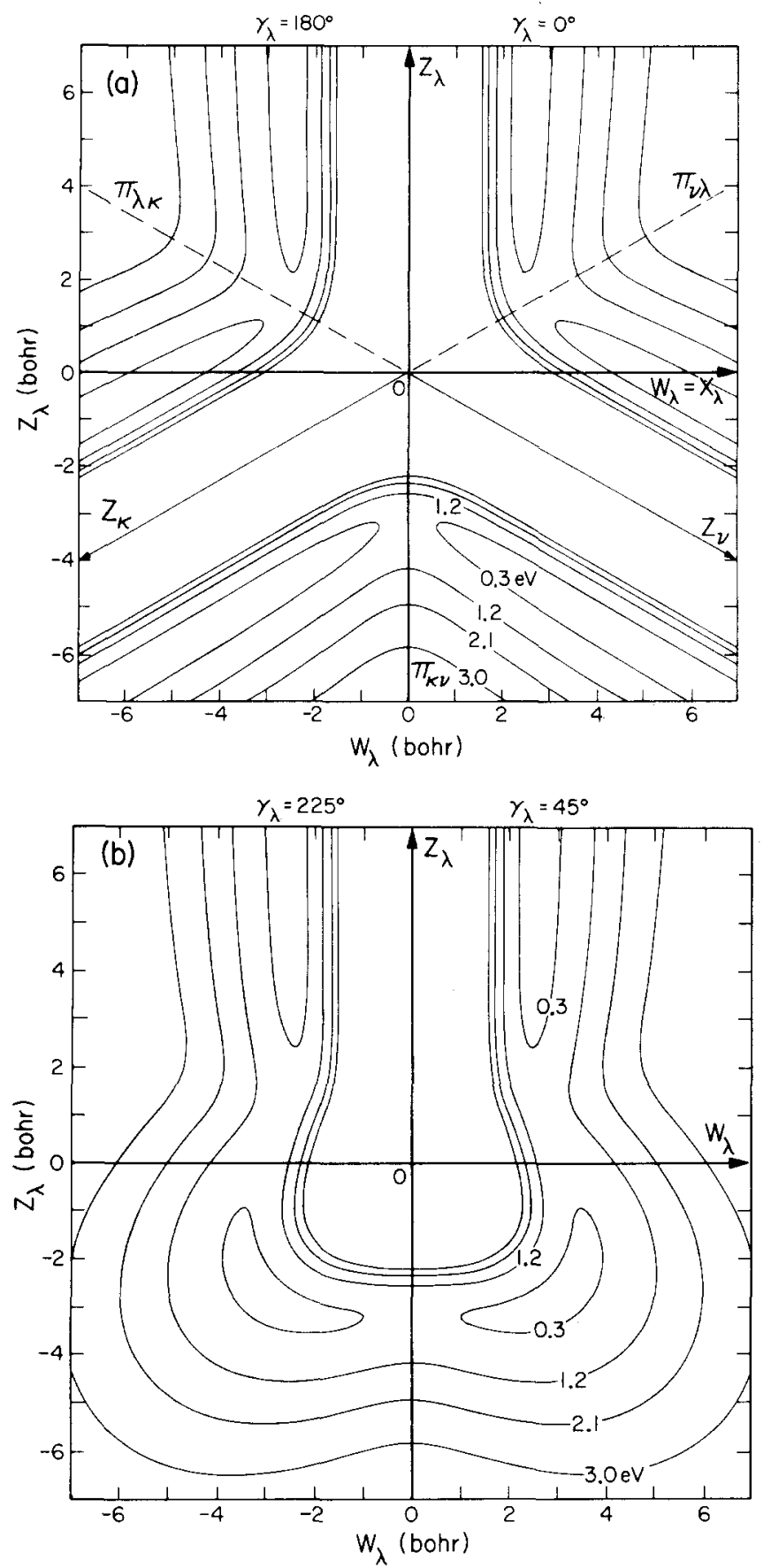

$j_{\lambda}$ associated with $\mathbf{r}_{\lambda}$ is defined as

$$
\mathrm{j}_{\lambda}=\frac{\hbar}{i}\left(\frac{\partial}{\partial \gamma_{\lambda}}\right)_{\theta_{\lambda}, R_{\lambda}, r_{\lambda}} \hat{\mathbf{z}},
$$

and its eigenfunctions are

$$
\varphi_{j_{\lambda}}\left(\gamma_{\lambda}\right)=(2 \pi)^{-1 / 2} \exp \left(i j_{\lambda} \gamma_{\lambda}\right) \quad j_{\lambda}=0, \pm 1, \pm 2, \ldots
$$

The expansion of $\psi_{J}^{\lambda}$, defined by Eq. $(2,13)$, in terms of these eigenfunctions is

$$
\psi_{J}^{\lambda}\left(r_{\lambda}, R_{\lambda}, \gamma_{\lambda}\right)=\sum_{j_{\lambda}} \varphi_{j_{\lambda}}\left(\gamma_{\lambda}\right) F_{J j_{\lambda}}^{\lambda}\left(r_{\lambda}, R_{\lambda}\right) 。
$$

Substituting this into Eq. (2.14), taking the scalar product of both sides by $\varphi_{j,}$, and interchanging the primed and unprimed quantum numbers, we obtain the following set of coupled partial differential equations in the two

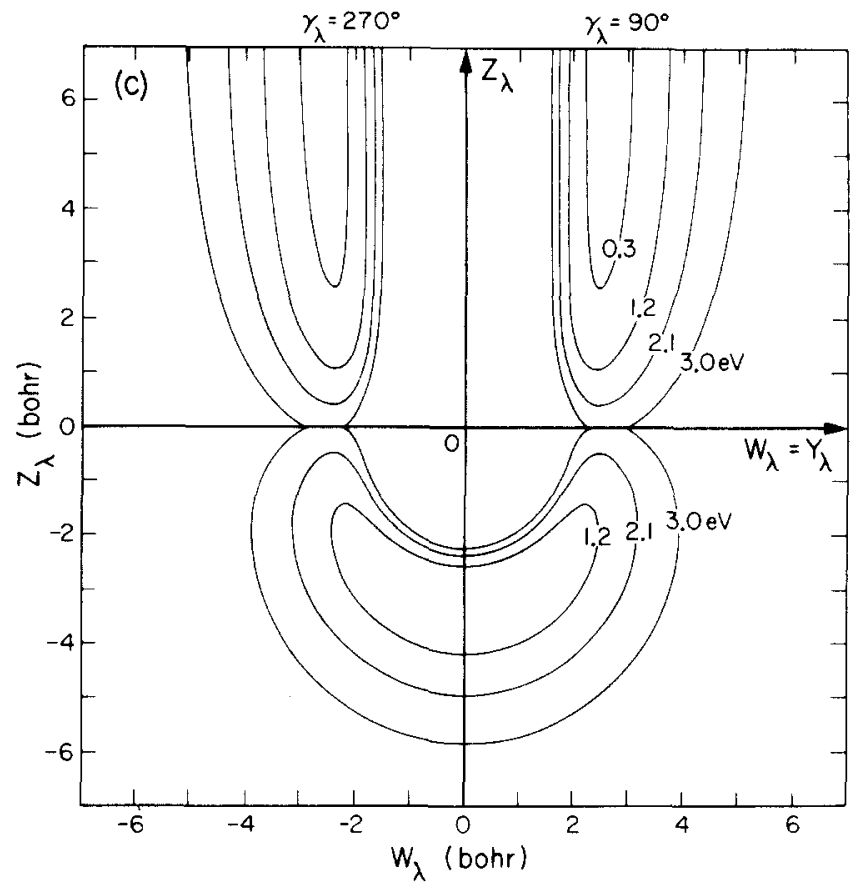

FIG. 2. Plot of potential contours for the $\mathrm{H}+\mathrm{H}_{2}$ reaction in the $O X_{\lambda} Y_{\lambda} Z_{\lambda}$ space defined in the text using the spherical polar coordinate mapping of Eq. (3.1), for six fixed values of the azimuthal angle $\gamma_{\lambda^{\circ}}$ (a) $\gamma_{\lambda}=0^{\circ}$ and $180^{\circ}$, (b) $\gamma_{\lambda}=45^{\circ}$ and $225^{\circ}$, (c) $\gamma_{\lambda}=90^{\circ}$ and $270^{\circ}$. In (a) we also depict the lines of intersection of the $\gamma_{\lambda}=0^{\circ}, 180^{\circ}$ plane with the half-planes $\pi_{\nu \lambda}, \pi_{\kappa \lambda}$, $\pi_{\lambda k}$ defined by Eq. (3.2), which are used to divide configuration space into three arrangement channel regions, $\lambda, \nu, \kappa_{0}$ For each figure above, $O W_{\lambda}$ is the intersection of the half-planes $\pi_{\nu \lambda}$, $\pi_{k \lambda}$, determined by $O Z_{\lambda}$ and the corresponding smallest $\gamma_{\lambda}$ with the $O X_{\lambda} Y_{\lambda}$ plane

scaled distances $r_{\lambda}, R_{\lambda}$ :

$$
\begin{aligned}
& -\frac{\hbar^{2}}{2 \mu}\left(\frac{\partial^{2}}{\partial r_{\lambda}^{2}}+\frac{\partial^{2}}{\partial R_{\lambda}^{2}}-\frac{j_{\lambda}^{2}-\frac{1}{4}}{r_{\lambda}^{2}}-\frac{\left(J-j_{\lambda}\right)^{2}-\frac{1}{4}}{R_{\lambda}^{2}}\right) F_{J j_{\lambda}}^{\lambda}\left(r_{\lambda}, R_{\lambda}\right) \\
& \quad+\sum_{j_{\lambda}^{\prime}=-\infty}^{\infty} V_{j_{\lambda} j_{\lambda}^{\prime}}^{\lambda}\left(r_{\lambda}, R_{\lambda}\right) F_{J j_{\lambda}^{\prime}}^{\lambda}\left(r_{\lambda}, R_{\lambda}\right) \\
& \quad=E F_{J j_{\lambda}}^{\lambda}\left(r_{\lambda}, R_{\lambda}\right) \quad J, j_{\lambda}=0, \pm 1, \pm 2, \ldots,
\end{aligned}
$$

where

$$
\begin{aligned}
V_{j_{\lambda} j_{\lambda}^{\prime}}^{\lambda}\left(r_{\lambda}, R_{\lambda}\right) & =\left\langle j_{\lambda}\left|V^{\lambda}\right| j_{\lambda}^{\prime}\right\rangle \\
& =\int_{0}^{2 \mathbf{r}} \varphi_{j_{\lambda}}^{\lambda^{*}}\left(\gamma_{\lambda}\right) V^{\lambda}\left(\gamma_{\lambda}, R_{\lambda}, \gamma_{\lambda}\right) \varphi_{j_{\lambda}^{\prime}}^{\lambda}\left(\gamma_{\lambda}\right) d \gamma_{\lambda} .
\end{aligned}
$$

Since $V^{\lambda}\left(\gamma_{\lambda}, R_{\lambda}, \gamma_{\lambda}\right)=V^{\lambda}\left(\gamma_{\lambda}, R_{\lambda}, 2 \pi-\gamma_{\lambda}\right)$, we can expand the potential in terms of a cosine Fourier series 


$$
V^{\lambda}\left(r_{\lambda}, R_{\lambda}, \gamma_{\lambda}\right)=\sum_{k=0}^{\infty} V_{k}^{\lambda}\left(r_{\lambda}, R_{\lambda}\right) \cos \left(k \gamma_{\lambda}\right),
$$

where

$$
V_{k}^{\lambda}\left(r_{\lambda}, R_{\lambda}\right)=\frac{2}{\left(1+\delta_{k 0}\right) \pi} \int_{0}^{\pi} \cos \left(k \gamma_{\lambda}\right) V^{\lambda}\left(r_{\lambda}, R_{\lambda}, \gamma_{\lambda}\right) d \gamma_{\lambda}
$$

Substituting Eq. $(3,8)$ into $(3,7)$ and integrating, we find that

$$
\begin{aligned}
V_{j_{\lambda} j_{\lambda}^{\prime}}^{\lambda}\left(r_{\lambda}, R_{\lambda}\right) & =\frac{1}{2-\delta_{\left|j_{\lambda}-j_{\lambda}^{\prime}\right|, 0}} V_{\left|j_{\lambda}-j_{\lambda}^{\prime}\right|}^{\lambda}\left(r_{\lambda}, R_{\lambda}\right) \\
& =\frac{1}{\pi} \int_{0}^{\pi} \cos \left(\left|j_{\lambda}-j_{\lambda}^{\prime}\right| \gamma_{\lambda}\right) V^{\lambda}\left(r_{\lambda}, R_{\lambda}, \gamma_{\lambda}\right) d \gamma_{\lambda} 。
\end{aligned}
$$

Equation (3.9) shows that $V_{j_{\lambda j \lambda} j_{\lambda}^{\prime}}$ depends on $j_{\lambda}$ and $j_{\lambda}^{\prime}$ only through $\left|j_{\lambda}-j_{\lambda}^{\prime}\right|$. We can write Eq。 $(3,6)$ in a condensed matrix notation by regarding $\mathbf{F}_{J}^{\lambda}\left(r_{\lambda}, R_{\lambda}\right)$ as a column vector whose elements are the $F_{J j_{\lambda}}^{\lambda}\left(r_{\lambda}, R_{\lambda}\right)$ 。 We get

$$
T^{\lambda} \mathbf{F}_{J}^{\lambda}+\mathbf{V}_{J}^{\lambda e} \mathbf{F}_{J}^{\lambda}=E \mathbf{F}_{J}^{\lambda},
$$

where $T^{\lambda}$ is the kinetic energy operator

$$
T^{\lambda}=-\frac{\hbar^{2}}{2 \mu}\left(\frac{\partial^{2}}{\partial R_{\lambda}^{2}}+\frac{\partial^{2}}{\partial r_{\lambda}^{\overline{2}}}\right)
$$

and $\mathbf{V}_{J}^{\lambda e}\left(r_{\lambda}, R_{\lambda}\right)$ is an effective potential energy matrix defined by

$$
\mathbf{V}_{J}^{\lambda e}\left(r_{\lambda}, R_{\lambda}\right)=\mathbf{V}^{\lambda}\left(r_{\lambda}, R_{\lambda}\right)+\mathbf{V}_{J}^{\lambda c}\left(r_{\lambda}, R_{\lambda}\right) 。
$$

$V^{\lambda}$ is the $J$-independent interaction potential matrix

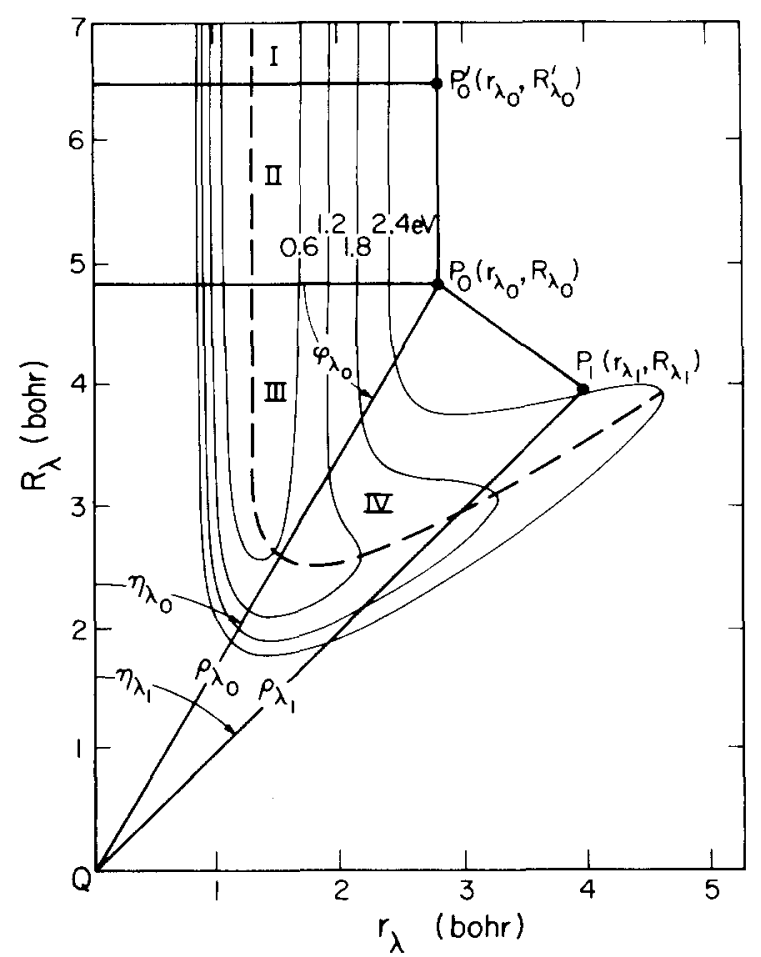

FIG. 3. Division of the $R_{\lambda}, r_{\lambda}$ space into four regions, I, II, III, and IV. The contours are equipotentials of the matrix element $V_{0}^{\lambda}\left(r_{\lambda}, R_{\lambda}\right)$ [see Eq. $(3,8)$ ] in $\mathrm{eV}$ for the Porter-Karplus $\mathrm{H}+\mathrm{H}_{2}$ potential energy function. The dashed line $L$ is the line of steepest ascents and descents for $V_{0}^{\lambda}$. The locations of the points $P_{0}^{\prime}, P_{0}$, and $P_{1}$ are discussed in Sec. III.C of the text. $Q$ is the origin of this space. whose elements are the $V_{j_{\lambda} j_{\lambda}^{\prime}}^{\lambda}$ defined by Eq. (3.9) while $\mathbf{V}_{\mathcal{J}}^{\lambda c}$ is the diagonal centrifugal potential matrix defined by

$$
\left[\mathbf{v}_{J}^{\lambda_{c}}\left(r_{\lambda}, R_{\lambda}\right)\right]_{j_{\lambda} j_{\lambda}^{\prime}}=\frac{\hbar^{2}}{2 \mu}\left(\frac{j_{\lambda}^{2}-\frac{1}{4}}{r_{\lambda}^{2}}+\frac{\left(J-j_{\lambda}\right)^{2}-\frac{1}{4}}{R_{\lambda}^{2}}\right) \delta_{j_{\lambda} j_{\lambda}^{j}} 。
$$

From $\operatorname{EqS}_{\circ}(3,6)$ and $(3,9)$ we conclude that the $F_{J j_{\lambda}}^{\lambda}$ for different $j_{\lambda}$ are coupled through the $V_{k}^{\lambda}\left(r_{\lambda}, R_{\lambda}\right)$ functions with $k>0, V_{0}^{\lambda}$ representing a noncoupling potential.

\section{The division of $r_{\lambda}, R_{\lambda}$ space into regions and the choice of variables in each region}

We now consider the expansion of the function $F_{J j_{\lambda}}^{\lambda}\left(r_{\lambda}\right.$, $R_{\lambda}$ ) in terms of a set of single-variable functions which describe a vibrationlike motion. To pick this vibration variable and the corresponding vibrational basis set, it is convenient to examine the behavior of $V_{0}^{\lambda}\left(r_{\lambda}, R_{\lambda}\right)$ since, as one can conclude from the remark at the end of Sec. III. B, this function together with the centrifugal potential $\left(\hbar^{2} / 2 \mu\right)\left(j_{\lambda}^{2}-\frac{1}{4}\right) / r_{\lambda}^{2}$ determines the $r_{\lambda}$ dependence of $F_{{ }_{j} j_{\lambda}}^{\lambda}$ in the absence of $j_{\lambda}$ (i。 $\mathrm{i}_{0}$, rotational) coupling. For the $\mathrm{H}+\mathrm{H}_{2}$ reaction, equipotentials of $V_{0}^{\lambda}\left(r_{\lambda}\right.$, $R_{\lambda}$ ) are represented in Fig。 3 , together with the corresponding line $L$ of steepest ascents and descents. Cuts of $V_{0}^{\lambda}$ normal to $L$ look like diatomic internuclear potential energy functions, displaying a minimum on $L$, a dissociation plateau in the direction away from the coordinate axes, and a steep repulsive point in the opposite direction. These characteristics are analogous to those presented by collinear triatomic potential energy functions and suggest that we divide the $r_{\lambda}, R_{\lambda}$ space in regions in a manner analogous to that employed for collinear reactions, ${ }^{5,10}$ using different coordinates and/or vibration basis functions in each region. The corresponding vibration coordinates will be a distance along appropriately chosen lines transverse (but not necessarily orthogonal) to $L$. In each region, in addition to such vibration coordinates, there will be a "propagation" coordinate in terms of which Eq. $(3,6)$, will be expressed as a system of coupled ordinary differential equations. For $\mathrm{H}+\mathrm{H}_{2}$ we indicate a convenient choice for these regions $\overline{i n}$ Fig. 3. They are denoted as follows: I-the asymptotic region; II-the weak interaction region; III-the strong interaction region; and IVthe matching region. The boundary points $P_{0}^{\prime}, P_{0}$, and $P_{1}$ in Fig。 3 are chosen as follows . The abscissa $r_{\lambda_{0}}$ of $P_{0}^{\prime}$ and $P_{0}$ is chosen so that these points lie in the classically forbidden plateau area where the wavefunction may be assumed to vanish. The ordinate $R_{\lambda_{0}}^{\prime}$ of $P_{0}^{\prime}$ is picked large enough so that the potential $V_{0}^{\lambda}\left(r_{\lambda}, R_{\lambda}\right)$ is independent of $R_{\lambda}$ for $R_{\lambda} \geqslant R_{\lambda_{0}{ }^{\circ}}^{\prime} \quad R_{\lambda_{0}}$ is determined by setting $\eta_{\lambda_{0}}$ of Fig. 3 equal to $\pi / 2-\alpha_{\nu \lambda} / 2$, from which we get

$$
R_{\lambda_{0}}=r_{\lambda_{0}} \tan \left(\alpha_{\nu \lambda} / 2\right)
$$

The reason for this choice is that in order to perform the matching of the different arrangement channel solutions, as described in Sec. III.A, we need the $F_{J j_{\lambda}}^{\lambda}$ defined by Eq. (3.5) for a range of values of $\eta_{\lambda}$. As justified after Eq. $(3.18)$, the lower limit of this range is the $\eta_{\lambda_{0}}$ just chosen. The same justification led to $\eta_{\lambda_{1}}$ 


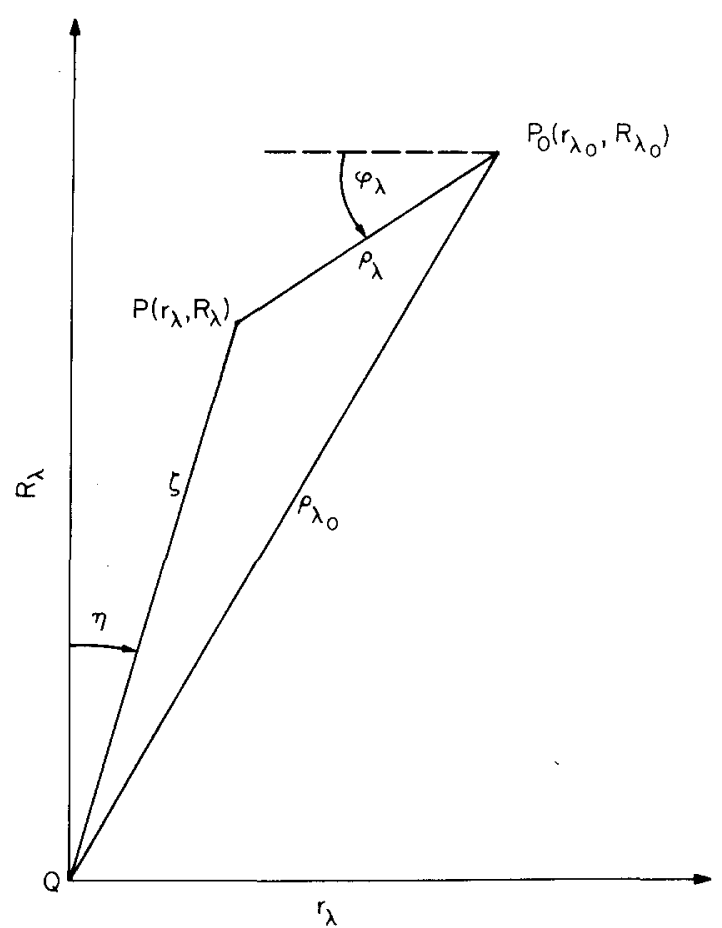

FIG. 4. The polar coordinates $\rho_{\lambda}, \varphi_{\lambda}$ and $\zeta, \eta_{\lambda}$ and their interrelationships in $R_{\lambda}, r_{\lambda}$ space.

$=\pi / 4$, from which we get (see Fig。3)

$$
R_{\lambda_{1}}=r_{\lambda_{1}} \text { 。 }
$$

This common value of $R_{\lambda_{1}}$ and $r_{\lambda_{1}}$ is chosen so that $P_{1}\left(r_{\lambda_{1}}, R_{\lambda_{1}}\right)$ also lies in the plateau region。

The coordinate systems used in each of these four regions are as follows. In the asymptotic region (I) and in the weak interaction region (II), we use the Cartesian coordinates $r_{\lambda}, R_{\lambda}$ as vibration and propagation coordinates, respectively. In the strong interaction region (III) we switch to the polar coordinates $\rho_{\lambda}, \varphi_{\lambda}$ (as depicted in Fig. 4) with $P_{0}\left(r_{\lambda 0}, R_{\lambda_{0}}\right)$ as origin and related to $r_{\lambda}, R_{\lambda}$ by

$$
\begin{aligned}
& r_{\lambda}=r_{\lambda_{0}}-\rho_{\lambda} \cos \varphi_{\lambda}, \\
& R_{\lambda}=R_{\lambda_{0}}-\rho_{\lambda} \sin \varphi_{\lambda} .
\end{aligned}
$$

$\rho_{\lambda}$ is the vibration and $\varphi_{\lambda}$ the propagation coordinate for this region. In the matching region (IV), we use another set of polar coordinates $\left(\zeta, \eta_{\lambda}\right)$ which have their origin at $Q$ (Fig. 4) and which are related to $r_{\lambda}, R_{\lambda}$ by

$$
\begin{aligned}
& r_{\lambda}=\zeta \sin \eta_{\lambda}, \\
& R_{\lambda}=\zeta \cos \zeta_{\lambda} .
\end{aligned}
$$

$\zeta(\geqslant 0)$ and $\eta_{\lambda}$ (in range $0-\pi / 2$ ) are, respectively, the vibration and propagation variables for Region IV. We note that the $\zeta$ defined here is identical to that given by Eq. (3.1a), while the angles $\omega_{\lambda}$ and $\eta_{\lambda}$ are related, as can be seen from Eqs. $(3.17)$ and $(3.1 b)$, by

$$
\omega_{\lambda}=2 \eta_{\lambda} \text { 。 }
$$

Once we know the $F_{J \delta_{\lambda}}^{\lambda}$ functions for a fixed $\eta_{\lambda}$ and variable $\zeta, E q_{0}(3.5)$ furnishes the wavefunction $\psi_{J}^{\lambda}$ on a cone of constant $\omega_{\lambda}\left(=2 \eta_{\lambda}\right)$ in the $O X_{\lambda} Y_{\lambda} Z_{\lambda}$ coordinate system, as depicted in Fig. 5(a). We actually want to determine this wavefunction on the matching surfaces $\pi_{\nu \lambda}$ and $\pi_{\lambda k}$ rather than on the $\omega_{\lambda}=$ constant cones since it is on these surfaces that we will smoothly match the solutions obtained from the integration of the Schrödinger equation in the three arrangements channels $\lambda=\alpha$, $\beta, \gamma$. However, for a given $\omega_{\lambda}$ (within a certain range) we do have the wavefunctions on the lines of intersection of the constant $\omega_{\lambda}$ cone with the half-planes $\pi_{\nu \lambda}$ and $\pi_{\lambda \kappa}$ [see Fig。 5(a)]. The relation between $\omega_{\lambda}$ and $\gamma_{\lambda}$ at the lines of intersection with $\pi_{\nu \lambda}$ is given by Eq。(A14) of Appendix A. As $\omega_{\lambda}$ is scanned from $\omega_{\lambda_{0}}=2 \eta_{0}$ to $\omega_{\lambda_{1}}$ $=2 \eta_{\lambda_{1}}$ (Figs. 3 and 5 ), these intersection lines scan the entire $\pi_{\nu \lambda}$ matching half-plane and thus we can obtain the desired wavefunction $\psi_{J}^{\lambda}$ on it from a knowledge of the $F_{J j_{\lambda}}^{\lambda}\left(\zeta, \eta_{\lambda}\right)$ for $\eta_{\lambda}$ in the range $\eta_{\lambda_{0}}$ to $\eta_{\lambda_{1}}$. The angles $\omega_{\lambda_{0}}$ and $\omega_{\lambda_{1}}$ correspond to the intersection of $\pi_{\nu \lambda}$ with the $\gamma_{\lambda}=0$ and $\gamma_{\lambda}=\pi / 2$ half-plane, respectively (in $O X_{\lambda} Y_{\lambda} Z_{\lambda}$ internal configuration space)。From $\mathrm{Eq}$. (A14) we find that $\omega_{\lambda_{0}}=\pi-\alpha_{\nu \lambda}$ and $\omega_{\lambda_{1}}=\pi / 2$. These values determine the values of $\eta_{\lambda_{0}}$ and $\eta_{\lambda_{1}}$ of Fig. 3 through Eq.

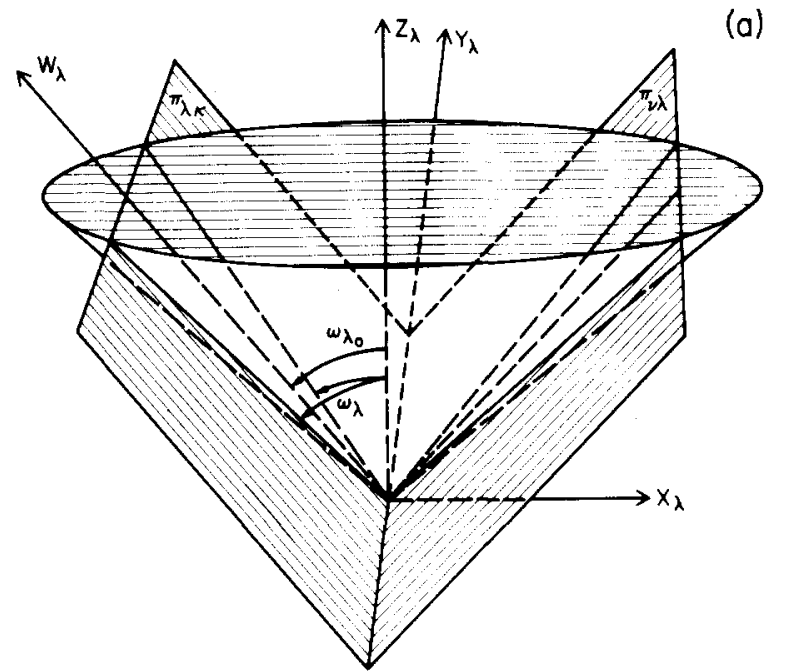

(a)

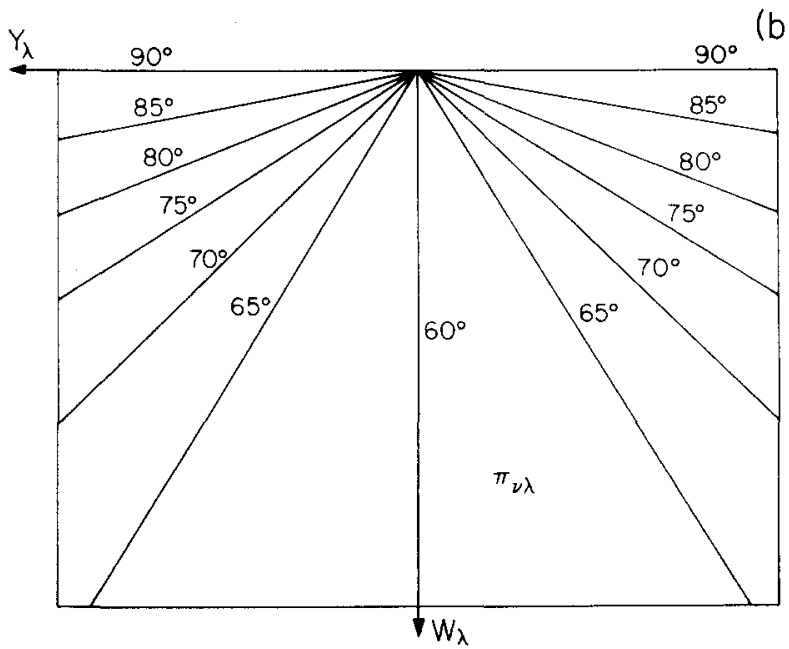

FIG. 5. (a) Plot of the matching half-planes $\pi_{\nu \lambda}$ and $\pi_{\lambda k}$, and their intersection with a cone of constant $\omega_{\lambda}\left(\omega_{\lambda_{0}} \leq \omega_{\lambda} \leq \omega_{\lambda 1}\right)$ showing the straight lines of intersection which occur at two angles $\gamma_{\lambda}\left(\omega_{\lambda}\right)$ for which $\cot \omega_{\lambda}=-\cot \alpha_{\nu \lambda} \cos \gamma_{\lambda}$, and two more for which $\cot \omega_{\lambda}=\cot \alpha_{\lambda k} \cos \gamma_{\lambda}$. (b) Lines of intersection of $\pi_{\nu \lambda}$ with constant $\omega_{\lambda}$ cones at intervals of $\Delta \omega_{\lambda}=5^{\circ}$ for $\mathrm{H}+\mathrm{H}_{2}$, where $\omega_{\lambda 0}=60^{\circ}$ and $\omega_{\lambda_{1}}=90^{\circ}$. 
(3.18), and consequently the positions of $P_{0}$ and $P_{1}$. Indeed, by using Eqs. (3.17) and (3.18) together with the values of $\omega_{\lambda_{0}}$ and $\omega_{\lambda_{1}}$ given above, we obtain the equations (3.14) and (3.15) that were previously used in locating $P_{0}$ and $P_{1}{ }^{32}$ One additional complication in this procedure arises when $m_{\nu} \neq m_{k}$ (for the integration in arrangement channel $\lambda$ ). In that case, the angles $\alpha_{\nu \lambda}$ and $\alpha_{\lambda k}$ are different [see Eq. (A3)] so that the value of $\omega_{\lambda_{0}}$ for $\pi_{\nu \lambda}\left(i_{0} e_{0}, \omega_{\lambda_{0}}=\pi-\alpha_{\nu \lambda}\right)$ is different from $\omega_{\lambda_{0}}^{\prime}$ for $\pi_{\lambda_{k}}\left(\omega_{\lambda_{0}}^{\prime}=\pi-\alpha_{\lambda_{k}}\right)$. In order to obtain the wavefunction on both of the matching surfaces $\pi_{\nu \lambda}$ and $\pi_{\nu \kappa}$, we modify our definition of $\omega_{\lambda_{0}}$ to

$$
\omega_{\lambda_{0}}=\min \left(\pi-\alpha_{\nu \lambda}, \pi-\alpha_{\lambda_{k}}\right) \text {. }
$$

$\omega_{\lambda_{1}}$, on the other hand, is mass independent and always equal to $\pi / 2$ for the choice of matching surfaces given by $E q .(3.2)$.

Having defined the four regions of each $r_{\lambda}, R_{\lambda}$ space $(\lambda=\alpha, \beta, \gamma)$ and their associated vibration-propagation coordinate systems, we are ready to introduce a vibrational expansion into the Schrödinger equations $\mathrm{Eq}$. (3.6) [or their matrix counterpart Eq。 $(3.10)$ ], thus obtaining the actual ordinary coupled differential equations to be integrated. We shall do this for each region separately, starting with the asymptotic region.

\section{The coupled Schrödinger equations in the propagation variable}

\section{The asymptotic region}

As mentioned above, the vibration and propagation variables for this region are $r_{\lambda}$ and $R_{\lambda}$, respectively. The function $V^{\lambda}\left(r_{\lambda}, R_{\lambda}, \gamma_{\lambda}\right)$ becomes the isolated diatomic potential $v^{\lambda}\left(r_{\lambda}\right)$ for $R_{\lambda} \geqslant R_{\lambda_{0}}^{\prime}$, and the potential matrix $V^{\lambda}$ appearing in the right hand side of Eq. $(3.12)$ becomes diagonal:

$$
\mathbf{V}^{\lambda}\left(r_{\lambda}, R_{\lambda} \geqslant R_{\lambda_{0}}\right)=v^{\lambda}\left(r_{\lambda}\right) \mathbf{I},
$$

where $I$ is the identity matrix. This leads to a total decoupling of Eqs. $(3,6)$ and $(3,10)$. For a given $J$ and $j_{\lambda}$, we obtain

$$
\begin{gathered}
{\left[-\frac{\hbar^{2}}{2 \mu}\left(\frac{\partial^{2}}{\partial r_{\lambda}^{2}}+\frac{\partial^{2}}{\partial R_{\lambda}^{2}}-\frac{\left(j_{\lambda}^{2}-\frac{1}{4}\right)}{r_{\lambda}^{2}}+\frac{\left(J-j_{\lambda}\right)^{2}-\frac{1}{4}}{R_{\lambda}^{2}}\right.\right.} \\
\left.\left.+v^{\lambda}\right)\right]_{\substack{\lambda(a) \\
J j_{\lambda}}}^{\lambda\left(E F_{j j_{\lambda}}^{\lambda(a)},\right.}
\end{gathered}
$$

where the superscript $(a)$ refers to the asymptotic region. This equation may be solved by separation of variables. The solution can be expanded as

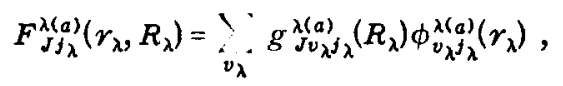

where $\phi_{v_{\lambda} j_{\lambda}}^{\lambda(a)}$ is a vibrational eigenfunction with vibrational energy $\epsilon_{v \lambda j_{\lambda}}^{\lambda(a)}$ satisfying the equation

$$
\left(-\frac{\hbar^{2}}{2 \mu} \frac{d^{2}}{d r_{\lambda}^{2}}+v^{\lambda}\left(r_{\lambda}\right)+\frac{j_{\lambda}^{2}-\frac{1}{4}}{r_{\lambda}^{2}}\right) \phi_{v_{\lambda}^{j} j_{\lambda}}^{\lambda(a)}=\epsilon_{v \lambda^{j} j_{\lambda}}^{\lambda(a)} \phi_{v_{\lambda}^{j} \lambda}^{\lambda(a)}
$$

with boundary conditions $\phi_{v_{\lambda} j_{\lambda}}^{\lambda(a)}(0)=\phi_{v_{\lambda} j_{\lambda}}^{\lambda(a)}\left(r_{\lambda_{0}}\right)=0$. $g_{J v_{\lambda} j_{\lambda}}^{\lambda(a)}\left(R_{\lambda}\right)$ describes the translational motion associated with the propagation variable, $R_{\lambda}$, and satisfies the equation

$$
\left.\left(\frac{d^{2}}{d R_{\lambda}^{2}}-\frac{\left(J-j_{\lambda}\right)^{2}-\frac{1}{4}}{R_{\lambda}^{2}}+k_{v_{\lambda}^{j} \lambda}^{\lambda(a)}\right)^{2}\right) g_{v \lambda \lambda_{\lambda}}^{\lambda(a)}=0
$$

where

$$
k_{v_{\lambda} j_{\lambda}}^{\lambda(a)^{2}}=\frac{2 \mu}{\hbar^{2}}\left(E-\epsilon_{v_{\lambda} j_{\lambda}}^{\lambda(a)}\right)
$$

is the square of the relative motion wave number when the diatom is in the vibration-rotation state labeled by the quantum numbers $v_{\lambda}, j_{\lambda^{\circ}}$ Equation $(3.24)$ is closely related to the Bessel equation, and its general solution can be written as

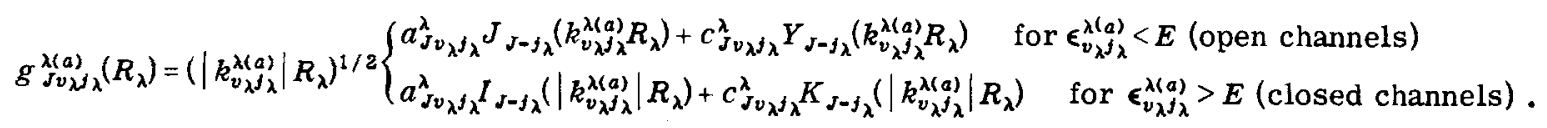

The functions $J_{l}$ and $Y_{l}$ are, respectively, the regular and irregular ordinary Bessel functions ${ }^{33}$ of order $l$ and have the asymptotic behavior

$$
\begin{aligned}
& x^{1 / 2} J_{l}(x) \underset{x \rightarrow \infty}{\sim}(2 / \pi)^{1 / 2} \cos (x-l \pi / 2-\pi / 4), \\
& x^{1 / 2} Y_{l}(x) \underset{x \rightarrow \infty}{\sim}(2 / \pi)^{1 / 2} \sin (x-l \pi / 2-\pi / 4),
\end{aligned}
$$

whereas $I_{l}$ and $K_{l}$ are the modified Bessel functions ${ }^{33}$ and have the asymptotic behavior

$$
\begin{aligned}
& x^{1 / 2} I_{l}(x) \underset{x=\infty}{\sim}(2 \pi)^{-1 / 2} \exp (x), \\
& x^{1 / 2} K_{l}(x) \underset{x \rightarrow \infty}{\sim}(\pi / 2)^{1 / 2} \exp (-x) .
\end{aligned}
$$

In Sec. $V$ we will consider scattering asymptotic conditions which will determine the constants $a_{J_{v_{\lambda} j_{\lambda}}^{\lambda}}$ and $c_{J \nu \lambda^{j} \lambda^{\circ}}^{\lambda}$

Let us rewrite Eqs. $(3.24)$ and $(3.26)$ in matrix notation, which will appreciably simplify the equations in the rest of the paper. Let $g_{J}^{\lambda(a)}\left(R_{\lambda}\right)$ be the column vec- tor whose elements, labeled by the $v_{\lambda} j_{\lambda}$ index pair, are given by Eq. (3.26). When replaced into Eqs. $(3.22)$, (3.5), and (2.13), it furnishes a single $\chi_{J}^{\lambda}\left(R_{\lambda}, r_{\lambda}, \gamma_{\lambda}\right)$, corresponding to a single $a_{J}^{\lambda}, c_{J}^{\lambda}$ column vector pair, the elements of which are the $a_{J_{v \lambda J_{\lambda}}^{\lambda}}$ and $c_{J_{v_{\lambda} j_{\lambda}}^{\lambda}}$ of Eq. (3.25). In order to satisfy Condition (c) of Sec. II.A, we will need a complete set of linearly independent solutions of the Schrödinger equation. The number of solutions in such a set is twice the number of $v_{\lambda} j_{\lambda}$ channels included in the expansions of Eqs. (3.22) and (3.5), which should be infinite but for practical reasons are truncated at a finite number $N_{0}$ (The errors introduced by this truncation will be discussed subsequently. ${ }^{20}$ ) We can assemble the corresponding $\mathbf{g}_{J}^{\lambda(a)}$ vectors to form two square matrices of dimension $N \times N$ which we shall label $\mathbf{g}_{J}^{\lambda(a)+}\left(R_{\lambda}\right)$ and $\mathbf{g}_{J}^{\lambda(a)-}\left(R_{\lambda}\right)$. In each of these two matrices, the rows will be labeled by $\left(v_{\lambda}, j_{\lambda}\right)$ pairs and the columns by $\left(v_{\lambda}^{\prime}, j_{\lambda}^{\prime}\right)$ pairs. In the course of our integration, we will obtain the solutions labeled $(+)$ by a 
propagation from Region I to IV and those labeled (-) by a propagation in the opposite direction. If we similarly form the matrices $a_{J}^{ \pm}$and $c_{J}^{ \pm}$from the corresponding $a_{J}^{*}$ and $c_{J}^{*}$ vectors, we can rewrite Eqs。 $(3,24)$ and $(3,26)$ as

$$
\frac{d^{2} \mathbf{g}_{J}^{\lambda(a) \pm}}{d R_{\lambda}^{2}}=\mathbf{U}_{J}^{\lambda(a)}\left(R_{\lambda}\right) \mathbf{g}_{J}^{\lambda(a) \pm}
$$

and

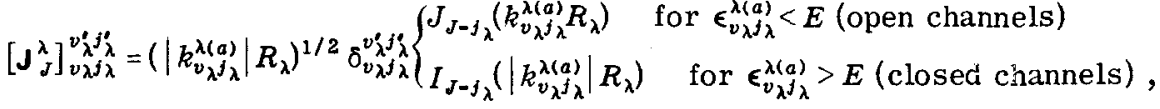

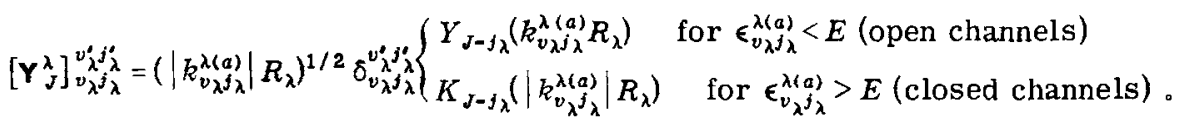

In Eqs. (3.32) through (3.35) the subscripts in a matrix element represent its row label and the superscripts its column label. Equations analogous to $\mathrm{Eq}$. $(3.29)$ will be developed for each of the other three subregions. Equation (3.30) and similar equations will be used in the reactance matrix analysis (Sec. V).

\section{The weak interaction region}

In this region, we retain the vibration-propagation variables $r_{\lambda}, R_{\lambda^{\circ}}$. However, since $V^{\lambda}\left(r_{\lambda}, R_{\lambda}, \gamma_{\lambda}\right)$ and $\mathbf{V}^{\lambda}\left(\boldsymbol{r}_{\lambda}, R_{\lambda}\right)$ do vary with $R_{\lambda}$, the asymptotic vibrational eigenfunctions $\phi_{v_{\lambda} j_{\lambda}}^{\lambda(a)}$ are not necessarily the best basis for expanding the $F_{J_{\lambda}}^{\lambda}\left(r_{\lambda}, R_{\lambda}\right)$. To optimize a choice of basis functions, we divide Region II into $n_{I I}^{\lambda}$ subregions $\Pi_{i}\left(i=1,2, \ldots, n_{1 \mathrm{I}}^{\lambda}\right)$ by constant $R_{\lambda}$ lines having $R_{\lambda}$ $=\left(R_{\lambda_{0}}^{\prime}, R_{\lambda_{1}}^{\prime}, \ldots, R_{\lambda_{n_{11}}}^{\prime}=R_{\lambda_{0}}\right)$. The range of $R_{\lambda}$ for the $i$ th subregion is $R_{\lambda_{i-1}^{\prime}}^{\prime} \stackrel{\text { II }}{\leq} R_{\lambda} \leq R_{\lambda_{i}}^{\prime}$. Let $R_{\lambda_{i}}^{0}$ be a selected value of $R_{\lambda}$ in this range, such as its midpoint. We choose as a basis set for expansion in this subregion the functions $\phi_{v_{\lambda_{j} j}}^{\lambda(w)}\left(r_{\lambda} ; R_{\lambda_{i}}^{0}\right)$ which satisfy the eigenvalue equation

$$
\begin{aligned}
& \left(-\frac{\hbar^{2}}{2 \mu} \frac{d^{2}}{d r_{\lambda}^{2}}+V_{\text {ref }}^{\lambda}\left(r_{\lambda} ; R_{\lambda_{i}}^{0}\right)+\frac{\left(j_{\lambda}^{2}-\frac{1}{4}\right) \hbar^{2}}{2 \mu r_{\lambda}^{2}}\right) \phi_{v_{\lambda} j_{\lambda}}^{\lambda(w)} \\
& =\epsilon_{v_{\lambda} J \lambda}^{\lambda(w)}\left(R_{\lambda_{i}}^{0}\right) \phi_{v_{\lambda} j_{\lambda}}^{\lambda(w)}
\end{aligned}
$$

with boundary conditions $\phi_{v_{\lambda}^{j} \lambda}^{\lambda(w)}\left(0 ; R_{\lambda_{i}}^{0}\right)=\phi_{v_{\lambda}^{j} \lambda_{\lambda}}^{\lambda(w)}\left(r_{\lambda_{0}} ; R_{\lambda_{i}}^{0}\right)$ $=0$. The superscript $(w)$ refers to the weak interaction region. $V_{\text {ref }}^{\lambda}\left(r_{\lambda} ; R_{\lambda_{i}}^{0}\right)$ is the value of a reference potential $V_{\text {ref }}^{\lambda}\left(r_{\lambda}, R_{\lambda}\right)$ at $R_{\lambda}=R_{\lambda_{i}}^{0}$. It is, in principle, arbitrary, but in practice must be chosen so that the vibrational basis functions $\phi_{v_{\lambda} j_{\lambda}}^{\lambda(w)}$ serve as an efficient representation of the $r_{\lambda}$ dependence of the functions $F_{J j_{\lambda}}^{\lambda}\left(r_{\lambda}\right.$, $\left.R_{\lambda}\right)$. Examples of possible reference potentials are (a) the first coefficient in the Fourier expansion [Eq. $(3.8)]$ $V_{0}^{\lambda}\left(r_{\lambda}, R_{\lambda}\right)$, and (b) the exact potential $V^{\lambda}\left(R_{\lambda}, r_{\lambda}, \bar{\gamma}_{\lambda}\right)$ for a fixed $\bar{\gamma}_{\lambda^{\circ}}$ One important test of vibrational convergence in our method is the invariance of the final re- sults to the choice of $V_{\text {rof. }}^{\lambda}$ Equation $(3,35)$ is the radial Schrödinger equation of a pseudomolecule whose interatomic potential is $V_{\mathrm{ref}}^{\lambda}\left(r_{\lambda} ; R_{\lambda_{i}}^{0}\right)$, characteristic of Subregion $\Pi_{i}$, rather than $v^{\lambda}\left(r_{\lambda}\right)$, which characterizes the asymptotic region. [Its centrifugal potential is, however, not the normal $j_{\lambda}\left(j_{\lambda}+1\right) \hbar^{2} / 2 \mu r_{\lambda}^{2}$ one.] The $\phi_{v_{\lambda} j_{\lambda}}^{\lambda(w)}$ and $\epsilon_{v_{\lambda} j_{\lambda}}^{\lambda(w)}$ are obtained by numerically solving $\mathbf{E q}$. (3. 36), ${ }^{34}$ subject to the boundary conditions just mentioned.

We now expand $F_{J_{j}}^{\lambda}\left(r_{\lambda}, R_{\lambda}\right)$ in terms of the above vibrational basis functions:

$$
F_{J j_{\lambda}}^{\lambda(w) \pm}\left(r_{\lambda}, R_{\lambda}\right)=\sum_{v_{\lambda}} g \underset{j v_{\lambda} j_{\lambda}}{\lambda(w) \pm}\left(R_{\lambda} ; R_{\lambda_{i}}^{0}\right) \phi_{v_{\lambda} j_{\lambda}}^{\lambda(w)}\left(r_{\lambda} ; R_{\lambda_{i}}^{0}\right)
$$

Substituting this into Eq. $(3,6)$, multiplying by $\phi_{v_{\lambda}^{j_{\lambda}}}\left(r_{\lambda}\right.$; $R_{\lambda_{i}}^{0}$ ) and integrating over $r_{\lambda}$, then interchanging $v_{\lambda}$ and $v_{\lambda}^{\prime}$ and expressing the resulting coupled equations in the matrix form of the previous section, we get

$$
\frac{d^{2} \mathbf{g}_{J}^{\lambda(w) \pm}}{d R_{\lambda}^{2}}=\mathbf{U}_{J}^{\lambda(w)}\left(R_{\lambda} ; R_{\lambda_{j}}^{0}\right) \mathbf{g}_{J}^{\lambda(w) \pm},
$$

where

$$
\mathbf{U}_{J}^{\lambda(w)}=-\mathbf{K}^{\lambda(w)^{2}}+\mathbf{U}_{J}^{c \lambda(w)}+\mathbf{U}_{p}^{\lambda(w)} .
$$

The diagonal matrices $K^{\lambda(w)^{2}}$ and $\mathbf{U}_{J}^{c \lambda(w)}$ are defined by Eqs. (3.32) and (3.33), respectively, with the superscript $(a)$ replaced by $(w)$, while the $J$-independent coupling "potential" matrix $\mathbf{U}_{p}^{\lambda(w)}\left(R_{\lambda} ; R_{\lambda_{i}}^{0}\right)$ (whose physical dimension is that of the square of a wave number) is given by

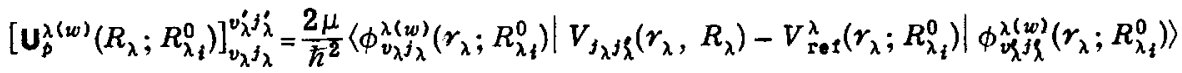

$$
\begin{aligned}
& =\frac{2 \mu}{\hbar^{2}}\left\langle\varphi_{j_{\lambda}}^{\lambda}\left(\gamma_{\lambda}\right) \phi_{v_{\lambda} j_{\lambda}}^{\lambda}\left(\gamma_{\lambda} ; R_{\lambda_{i}}^{0}\right)\left|V^{\lambda}\left(\gamma_{\lambda}, R_{\lambda}, \gamma_{\lambda}\right)-V_{r e f}^{\lambda}\left(\gamma_{\lambda} ; R_{\lambda_{i}}^{0}\right)\right| \phi_{v_{\lambda}^{j} j_{\lambda}^{j}}^{\lambda(w)}\left(r_{\lambda} ; R_{\lambda_{i}}^{0}\right) \varphi_{j_{\lambda}^{j}}^{\lambda}\left(\gamma_{\lambda}\right)\right\rangle \\
& =\frac{2 \mu}{\hbar^{2}}\left\langle v_{\lambda} j_{\lambda}\left|V^{\lambda}\left(r_{\lambda}, R_{\lambda}, \gamma_{\lambda}\right)-V_{r_{\text {ef }}}^{\lambda}\left(r_{\lambda} ; R_{\lambda_{i}}^{0}\right)\right| v_{\lambda}^{\prime} j_{\lambda}^{\prime}\right\rangle \text {. }
\end{aligned}
$$


As long as the anisotropy of the potential remains small and the reference potential provides an efficient vibrational basis set for expanding the functions $F_{J J_{\lambda}}^{\lambda}\left(r_{\lambda} ; R_{\lambda}\right)$ within each subregion, the matrix $\mathbf{U}_{J}^{\lambda(w)}$ should be nearly diagonal, and Eqs. (3.38) are only weakly coupled. The number and size of the subregions into which Region II is divided depends on the steepness of the variation of the potential matrix $\left\langle v_{\lambda} j_{\lambda}\left|V^{\lambda}\right| v_{\lambda}^{\prime} j_{\lambda}^{\prime}\right\rangle$ with $R_{\lambda}$. This number is generally small (i.e., less than 3 ).

As we propagate the solutions of Eq. (3.38) through Region II towards Regions I or III, the wavefunctions $\chi_{j}^{\lambda}\left(r_{\lambda}, R_{\lambda}, \gamma_{\lambda}\right)$ must remain continuous and smooth during the change of basis functions that occurs at the boundaries of neighboring subregions. This requirement leads to the following relations between the functions

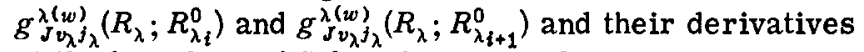
at the boundary of Subregions $\Pi_{i}$ and $\mathrm{II}_{i+1}$ :

$$
\begin{aligned}
& \boldsymbol{\theta}_{J}^{\lambda(w) \pm}\left(R_{\lambda_{i}}^{\prime} ; R_{\lambda_{i+1}}^{0}\right)=\mathbf{S}_{i}^{\lambda(w)} \mathbf{g}_{J}^{\lambda(w) \pm}\left(R_{\lambda_{i}}^{\prime} ; R_{\lambda_{i}}^{0}\right), \\
& \frac{d \mathbf{g}_{j}^{\lambda(w) \pm}}{d R_{\lambda}}\left(R_{\lambda_{i}}^{\prime} ; R_{\lambda_{i+1}}^{0}\right)=\mathbf{S}_{i}^{\lambda(w)} \frac{d \mathbf{g}_{j}^{\lambda}(w) \pm\left(R_{\lambda_{i}}^{\prime} ; R_{\lambda_{i}}\right)}{d R_{\lambda}},
\end{aligned}
$$

where the overlap matrix $\mathbf{S}_{i}^{\lambda(w)}$ is given by $\left[\mathbf{S}_{i}^{\lambda(w)}\right]_{v_{\lambda}^{j} j_{\lambda}}^{v^{* j} \dot{j}}=\delta_{j_{\lambda} j \lambda}\left\langle\phi_{v_{\lambda} j_{\lambda}}^{\lambda(w)}\left(r_{\lambda} ; R_{\lambda_{i+1}}^{0}\right) \mid \phi_{v_{\lambda}^{\prime j} j_{\lambda}^{j}}^{\lambda(w)}\left(r_{\lambda} ; R_{\lambda_{i}}^{0}\right)\right\rangle$.

An important criterion for the choice of the size of the subregions is that the $\boldsymbol{S}_{i}^{\lambda(w)}$ should be nearly orthogonal matrices. Lack of orthogonality implies lack of completeness of the basis sets $\phi_{v_{\lambda} j_{\lambda}}^{\lambda(w)}$ and results in the loss of information about the wavefunction as a result of the transformation of Eqs. (3.41). The relations at the boundary separating Regions I and II are achieved by setting $i=0$ in Eqs. (3.41) and interpreting $R_{\lambda_{0}}^{0}$ to mean $R_{\lambda_{0}}^{\prime}$ and $\phi_{v_{\lambda^{j}}{ }^{j}}^{\lambda(w)}\left(r_{\lambda} ; R_{\lambda_{0}}^{0}\right)$ to mean $\phi_{v_{\lambda} j_{\lambda}}^{\lambda(a)}\left(r_{\lambda}\right)$.

\section{The strong interaction region}

In this region we use the polar coordinates $\rho_{\lambda}, \varphi_{\lambda}$ regarding $\varphi_{\lambda}$ as the propagation variable. If we transform Eq. (3.10) to these polar coordinates with the aid of $\mathrm{Eq}$. (3.16), we obtain the vector equation

$$
T^{\lambda(s)} F_{J}^{\lambda(s)}\left(\rho_{\lambda}, \varphi_{\lambda}\right)+V_{J}^{\lambda(s)} F_{J}^{\lambda(s)}=E F_{J}^{\lambda(s)},
$$

where the superscript $(s)$ refers to "strong interaction region." $T^{\lambda^{(s)}}$ is given by

$$
T^{\lambda(s)}=-\frac{\hbar^{2}}{2 \mu}\left(\frac{\partial^{2}}{\partial \rho_{\lambda}^{2}}+\frac{1}{\rho_{\lambda}} \frac{\partial}{\partial \rho_{\lambda}}+\frac{1}{\rho_{\lambda}^{2}} \frac{\partial^{2}}{\partial \varphi_{\lambda}^{2}}\right),
$$

and the matrix $\mathbf{V}_{J}^{\lambda e(s)}\left(\rho_{\lambda}, \varphi_{\lambda}\right)$ is simply $\mathbf{V}_{J}^{\lambda e}\left(r_{\lambda}, R_{\lambda}\right)$ expressed in the coordinates $\rho_{\lambda}, \varphi_{\lambda}$.

For a typical reference potential $V_{\text {ros }}^{\lambda}$ such as the $V_{0}^{\lambda}\left(r_{\lambda}, R_{\lambda}\right)$ defined by Eq. (3. 8b) and plotted in Fig. 3 for $\mathrm{H}+\mathrm{H}_{2}$, the shape of the potential as a function of $\rho_{\lambda}$ for a fixed $\varphi_{\lambda}\left(0 \leq \varphi_{\lambda} \leq \varphi_{\lambda_{0}}\right)$ is very much like that for a diatomic molecule. The deep potential well character of these cuts at constant $\varphi_{\lambda}$ permits one to use their bound state eigenfunctions to efficiently represent the $\rho_{\lambda}$ dependence of the wavefunction in this region. Accordingly, we divide Region III into $n_{\mathrm{III}}^{\lambda}$ subregions $\mathrm{III}_{i}(i=1$, $2, \ldots, n_{1 \mathrm{II}}^{\lambda}$ ) by lines of constant

$$
\varphi_{\lambda}\left(\varphi_{\lambda}=0, \varphi_{\lambda_{1}}^{\prime}, \varphi_{\lambda_{2}}^{\prime}, \ldots, \varphi_{\lambda_{n_{1 I I}}^{\prime}}^{\prime}=\varphi_{\lambda_{0}}\right) \text {. }
$$

For each such subregion, we choose a cut at a specific value $\varphi_{\lambda_{i}}^{0}$ of $\varphi_{\lambda}$ (where $\varphi_{\lambda_{i-1}} \leq \varphi_{\lambda_{i}}^{0} \leq \varphi_{\lambda_{i}}$ ) to define our vibrational basis functions $\phi_{\nu_{\lambda}}^{\lambda_{\lambda}(s)}\left(\rho_{\lambda} ; \varphi_{\lambda_{i}}^{0}\right)$ and energy eigenvalues $\epsilon_{v_{\lambda}}^{\lambda(s)}\left(\varphi_{\lambda_{l}}^{0}\right)$ as the solutions of the Schrödinger equation:

$\left(-\frac{\hbar^{2}}{2 \mu} \frac{d^{2}}{d \rho_{\lambda}^{2}}+V_{r \in f}^{\lambda}\left(\rho_{\lambda} ; \varphi_{\lambda_{i}}^{0}\right)\right) \phi_{v_{\lambda}}^{\lambda(s)}\left(\rho_{\lambda} ; \varphi_{\lambda_{i}}^{0}\right)=\epsilon_{v_{\lambda}}^{\lambda(s)}\left(\varphi_{\lambda_{i}}^{0}\right) \phi_{v_{\lambda}}^{\lambda(s)}$,

satisfying the boundary conditions $\phi_{v_{\lambda}}^{\lambda(s)}\left(\rho_{\lambda}=0\right)=\phi_{v_{\lambda}}^{\lambda(s)}\left(\rho_{\lambda}\right.$ $\left.=\rho_{\lambda_{i}}^{\prime}\right)=0$, where $\rho_{\lambda_{i}}^{\prime}$ is the value of $\rho_{\lambda}$ at the intersection of the line $\varphi_{\lambda}=\varphi_{\lambda_{i}}^{0}$ with the $R_{\lambda}$ axis (Fig. 3). The potential $V_{\text {ref }}^{\lambda}\left(\rho_{\lambda}, \varphi_{\lambda}\right)$ is one of the reference potentials $V_{r \in \mathcal{I}}^{\lambda}\left(r_{\lambda}, R_{\lambda}\right)$ defined in Sec. III. D. 2 expressed in $\rho_{\lambda}, \varphi_{\lambda}$ coordinates. Note that we have not included any $j_{\lambda}$-dependent centrifugal terms in the Hamiltonian of Eq. (3.45) (all of these terms being included in the "propagation part" of the Hamiltonian), and hence our eigenvalues and eigenfunctions depend only on the vibrational quantum number $v_{\lambda}$. This is done for computational convenience and does not seriously decrease the rate of convergence of our coupled-channel expansion.

We now expand the solutions $\mathrm{F}_{J}^{\lambda_{J}^{(s)}}$ of $\mathrm{Eq} .(3.43)$ in terms of the pseudovibrational functions $\phi_{v_{\lambda}}^{\lambda(s)}$,

$$
F_{J J_{\lambda}}^{\lambda(s) \star}\left(\rho_{\lambda}, \varphi_{\lambda}\right)=\rho_{\lambda}^{-1 / 2} \sum_{v_{\lambda}} g_{J \nu_{\lambda} j_{\lambda}}^{\lambda(s) \pm}\left(\varphi_{\lambda} ; \varphi_{\lambda_{f}}^{0}\right) \phi_{v_{\lambda}}^{\lambda(s)}\left(\rho_{\lambda} ; \varphi_{\lambda_{i}}^{0}\right) .
$$

The counterpart of Eq. (3.38) becomes

$$
\frac{d^{2} \mathbf{g}_{J}^{\lambda(s) \pm}}{d \varphi_{\lambda}^{2}}=\overline{\mathbf{U}}_{J}^{\lambda(s)}\left(\varphi_{\lambda} ; \varphi_{\lambda_{i}}^{0}\right) \mathbf{g}_{J}^{\lambda(s) \pm},
$$

where the effective "potential" matrix $\overline{\mathbf{U}}_{J}^{\lambda(s)}$ (whose elements are dimensionless) can be written as

$$
\overline{\mathbf{U}}_{J}^{\lambda(s)}=\rho_{\lambda}^{2}\left(\varphi_{\lambda_{i}}^{0}\right) \mathbf{U}_{J}^{\lambda(s)}\left(\varphi_{\lambda} ; \varphi_{\lambda_{i}}^{0}\right)
$$

in which

$$
\mathbf{U}_{J}^{\lambda(s)}=-\mathbf{K}^{\lambda(s)^{2}}+\mathbf{U}_{J}^{c \lambda(s)}+\mathbf{U}_{p}^{\lambda(s)} .
$$

The matrix $\rho_{\lambda}^{2}$ is given by

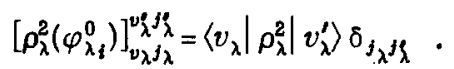

The matrix $\mathrm{K}^{\lambda(s)^{2}}$ and the "potential" coupling matrix $\mathbf{U}_{p}^{\lambda(s)}$ are quite similar to those in Eqs. (3.32) and (3.40) and are given by

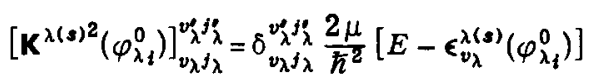

and

$$
\begin{aligned}
& {\left[\mathbf{U}_{\phi}^{\lambda(s)}\left(\varphi_{\lambda} ; \varphi_{\lambda_{1}}^{0}\right)\right]_{v_{\lambda} \lambda_{\lambda}}^{v_{\lambda}^{\prime j} j_{\lambda}^{j}}=\frac{2 \mu}{\hbar^{2}}\left\langle\phi_{v_{\lambda}}^{\lambda(s)}\left(\rho_{\lambda} ; \varphi_{\lambda_{i}}^{0}\right)\right| V_{j_{\lambda} j \ell}^{\lambda}\left(\rho_{\lambda}, \varphi_{\lambda}\right)} \\
& -V_{\mathrm{rof}}^{\lambda}\left(\rho_{\lambda} ; \varphi_{\lambda_{i}}^{0}\right)\left|\phi_{v_{\lambda}^{\prime}}^{\lambda(s)}\left(\rho_{\lambda} ; \varphi_{\lambda_{i}}^{0}\right)\right\rangle
\end{aligned}
$$

respectively, but the centrifugal potential matrix is now defined as

$$
\begin{aligned}
{\left[\mathbf{U}_{J}^{c(s)}\left(\varphi_{\lambda} ; \varphi_{\lambda_{i}}^{0}\right)\right]_{v_{\lambda} j_{\lambda}}^{v j_{j}^{j} \lambda}=} & \left\langle v_{\lambda}\right| \frac{j_{\lambda}^{2}-\frac{1}{4}}{\left(r_{\lambda_{0}}-\rho_{\lambda} \cos \varphi_{\lambda}\right)^{2}} \\
& +\frac{\left(J-j_{\lambda}\right)^{2}-\frac{1}{4}}{\left(R_{\lambda_{0}}-\rho_{\lambda} \sin \varphi_{\lambda}\right)^{2}}-\frac{1}{4 \rho_{\lambda}^{2}}\left|v_{\lambda}^{\prime}\right\rangle \delta_{j_{\lambda} j_{\lambda}^{\prime}},
\end{aligned}
$$

where the first term between vertical bars in Eq. (3.52) 
arises from the rotation of the diatom, the second from rotation of the atom-diatom system, and the third is a pseudocentrifugal potential arising from the use of the $\rho_{\lambda}, \varphi_{\lambda}$ coordinates and associated with the nonphysical swinging motion around the point $P_{0}$ of Fig. 3 as $\varphi_{\lambda}$ is changed. The centrifugal coupling $[\mathrm{Eq} .(3.52)]$ is still diagonal in $j_{\lambda}$ [ as is its counterpart for the $(a)$ and $(w)$ regions] but no longer in $v_{\lambda}$. In addition to the usual potential coupling resulting from the off-diagonal terms of $\mathbf{U}_{p}^{\lambda(s)}$, we also have coupling arising from the nondiagonal $\rho_{\lambda}^{2}$ matrix which appears in Eq. (3.48) as a result of the swinging motion around $P_{0}$. The neglect of this latter coupling could introduce serious errors since $\rho_{\lambda}^{2}$ has large off-diagonal elements. Such an approximation has nevertheless often been used, ${ }^{14,15}$ since it is one of the consequences of the one-vibrational-basisfunction approximation. Note also that the effective potential matrix $\overline{\mathbf{U}}_{J}^{\lambda(s)}$ is not symmetric, but is the product of two symmetric matrices as can be seen from Eq. (3.48). In Sec. III. E we discuss the Gordon method $^{35}$ used to integrate Eq. (3.47) and the modifications of it which are required when dealing with matrices of this type.

The smooth matching across the boundary separating any two neighboring subregions $\mathrm{III}_{i}$ and $\mathrm{III}_{i+1}$ is achieved through expressions analogous to Eqs. (3.41) in which the overlap matrix $\mathbf{S}_{i}^{\lambda}$ is given by

$$
\left[\mathbf{S}_{i}^{\lambda}\left(\varphi_{\lambda_{i}}^{0}\right)\right]_{v_{\lambda}^{j} \lambda_{\lambda}}^{v_{\lambda^{j} j_{\lambda}^{\prime}}^{j}}=\delta_{j_{\lambda^{j}} j_{\lambda}^{\prime}}\left\langle\phi_{v_{\lambda}}^{\lambda(s)}\left(\rho_{\lambda} ; \varphi_{\lambda_{i+1}}^{0}\right) \mid \phi_{v_{\lambda}^{\prime}}^{\lambda(s)}\left(\rho_{\lambda} ; \varphi_{\lambda_{i}}^{0}\right)\right\rangle
$$

and has elements which are independent of $j_{\lambda}, j_{\lambda}^{\prime}$. Since the general characteristics of the $\rho_{\lambda}$ dependence of the reference potential $V_{\text {ret }}^{\lambda}\left(\rho_{\lambda}, \varphi_{\lambda}\right)$ usually change quite rapidly as $\varphi_{\lambda}$ is changed, the number of subregions in Region III required to keep $\mathbf{S}_{i}^{\lambda}$ nearly orthogonal for a truncated vibrational basis set is generally large (on the order of $20-30$ ).

The smooth matching of the wavefunction $\chi_{j}^{\lambda}$ across the boundary between Regions $\Pi$ and $I I$ is achieved by the relations

$$
\mathbf{g}_{J}^{\lambda(s) \pm}\left(\varphi_{\lambda}=0 ; \varphi_{\lambda_{i}}^{0}\right)=\rho_{\lambda}^{1 / 2} \boldsymbol{g}_{J}^{\lambda(w) \pm}\left(R_{\lambda_{0}} ; R_{\lambda_{n_{11}^{\lambda}}^{0}}^{0}\right)
$$

and

$$
\frac{d \mathbf{g}_{J}^{\lambda(s) \pm}}{d \varphi_{\lambda}}\left(\varphi_{\lambda}=0 ; \varphi_{\lambda_{i}}^{0}\right)=-\rho_{\lambda}^{3 / 2} \frac{d \mathbf{g}_{J}^{\lambda(w) \pm}}{d R_{\lambda}}\left(R_{\lambda_{0}} ; R_{\lambda_{\lambda_{11}}}^{0}\right)
$$

where

$$
\begin{gathered}
\left(\rho_{\lambda}^{b}\right)_{v_{\lambda}^{j} \lambda}^{v^{j} j_{\lambda}^{j}}=\left\langle\phi_{v_{\lambda}}^{\lambda(s)}\left(\rho_{\lambda} ; \varphi_{\lambda}^{0}\right)\left|\rho_{\lambda}^{b}\right| \phi_{v_{\lambda}^{\prime j} \lambda}^{\lambda(w)}\left(r_{\lambda_{0}}-\rho_{\lambda} ; R_{\lambda_{n_{1} \lambda}^{0}}^{0}\right)\right\rangle \delta_{j_{\lambda}^{j} \lambda_{\lambda}^{\prime}} \\
b=\frac{1}{2}, \frac{3}{2} .
\end{gathered}
$$

\section{The matching region}

As described in Sec. III. C, the coordinates used in this region are the polar variables $\zeta, \eta_{\lambda}$ depicted in Fig. 4 , with $\eta_{\lambda}$ being the propagation variable. The Schrödinger equation in Region IV is very similar to that in Region III [see Eqs. (3.43)-(3.52)] with $\rho_{\lambda}, \varphi_{\lambda}$ replaced by $\zeta, \eta_{\lambda}$. From Eqs. (3.17) and (3.10) we obtain the counterpart of Eq. (3.43):

$$
T^{\lambda(m)} F_{J}^{\lambda(m)}\left(\zeta, \eta_{\lambda}\right)+V_{J}^{\lambda \rho(m)}\left(\zeta, \eta_{\lambda}\right) F_{J}^{\lambda(m)}=E F_{J}^{\lambda(m)}
$$

where

$$
T^{\lambda(m)}=-\frac{\hbar^{2}}{2 \mu}\left(\frac{\partial^{2}}{\partial \zeta^{2}}+\frac{1}{\zeta} \frac{\partial}{\partial \zeta}+\frac{1}{\zeta^{2}} \frac{\partial^{2}}{\partial \eta_{\lambda}^{2}}\right) .
$$

The superscript $(m)$ denotes "matching region," and $\mathbf{V}_{J}^{\lambda\left({ }^{(m)}\right.}\left(\zeta, \eta_{\lambda}\right)$ is obtained from $\mathbf{V}_{J}^{\lambda e}\left(r_{\lambda}, R_{\lambda}\right)$ by using Eq. (3.17). The division of Region IV into subregions is analogous to that for Regions II and III, and the vibrational basis functions $\phi_{v_{\lambda}}^{\lambda(m)}\left(\zeta ; \eta_{\lambda_{i}}^{0}\right)$ with eigenvalue $\epsilon_{v_{\lambda}}^{\lambda(m)}\left(\eta_{\lambda_{i}}^{0}\right)$ satisfy the equation

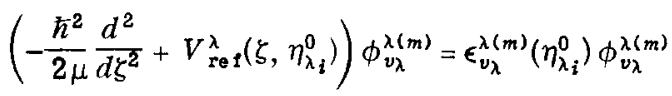

with boundary conditions $\phi_{v_{\lambda}}^{\lambda(m)}(0)=\phi_{\nu_{\lambda}}^{\lambda(m)}\left(\zeta=\zeta_{0}\right)=0$. The potential $V_{\mathrm{ref}}^{\lambda}\left(\zeta, \eta_{\lambda}\right)$ is one of the reference potentials $V_{\mathrm{re} \text { I }}^{\lambda}$ defined in Sec. III. D. 2 and III. D. 3 but expressed in $\zeta, \eta_{\lambda}$ coordinates. Writing

$$
F_{J j_{\lambda}}^{\lambda(m)}=\zeta^{-1 / 2} \sum_{v_{\lambda}} g_{J v_{\lambda^{j}}}^{\lambda(m)}\left(\eta_{\lambda} ; \eta_{\lambda_{i}}^{0}\right) \phi_{v_{\lambda}}^{\lambda(m)}\left(\zeta ; \eta_{\lambda_{i}}^{0}\right),
$$

the counterpart of $\mathrm{Eq}$. (3.47) becomes

$$
\frac{d^{2} \boldsymbol{g}_{J}^{\lambda(m) \pm}}{d \eta_{\lambda}^{2}}=\overline{\mathbf{U}}_{J}^{\lambda(m)}\left(\eta_{\lambda} ; \eta_{\lambda_{i}}^{0}\right) \mathbf{g}_{J}^{\lambda(m) \pm},
$$

where the effective potential matrix (again being physically dimensionless) is

$$
\overline{\mathbf{U}}_{J}^{\lambda(m)}=\zeta^{2}\left(\eta_{\lambda_{i}}^{0}\right) \mathbf{U}_{J}^{\lambda(m)}\left(\eta_{\lambda} ; \eta_{\lambda_{i}}^{0}\right)
$$

with

$$
\mathbf{U}_{J}^{\lambda(m)}\left(\eta_{\lambda} ; \eta_{\lambda i}^{0}\right)=-\mathbf{K}^{\lambda(m)^{2}}+\mathbf{U}_{J}^{c \lambda(m)}+\mathbf{U}_{p}^{\lambda(m)} .
$$

The matrix $\zeta^{2}\left(\eta_{\lambda i}^{0}\right)$ is defined analogously to $\rho_{\lambda}^{2}$ of Eq. (3.49) with $\rho_{\lambda}$ replaced by $\zeta$ and $\varphi_{\lambda}$ by $\eta_{\lambda}$. The matrices $\mathbf{K}^{\lambda(m)^{2}}$ and $\mathbf{U}_{p}^{\lambda(m)}$ are given by expressions similar to Eqs. (3.50) and (3.51), respectively, with the superscript $(m)$ replacing $(s)$ and appropriate coordinate changes made, while the centrifugal potential matrix is given by

$$
\begin{aligned}
& {\left[\mathbf{u}_{J}^{c \lambda(m)}\left(\eta_{\lambda} ; \eta_{\lambda i}^{0}\right)\right]_{v_{\lambda}^{j} \lambda_{\lambda}}^{v_{\lambda}^{j} j_{\lambda}}} \\
& \quad=\left\langle v_{\lambda}\left|\frac{j_{\lambda}^{2}-\frac{1}{4}}{\zeta^{2} \sin ^{2} \eta_{\lambda}}+\frac{\left(J-j_{\lambda}\right)^{2}-\frac{1}{4}}{\zeta^{2} \cos ^{2} \eta_{\lambda}}-\frac{1}{4 \zeta^{2}}\right| v_{\lambda}^{\prime}\right\rangle \delta_{j_{\lambda}^{j} \lambda} .
\end{aligned}
$$

Smooth transformation between subregions in Region IV involves Eqs. (3.41) with the overlap matrix $S_{i}^{\lambda}$ given by

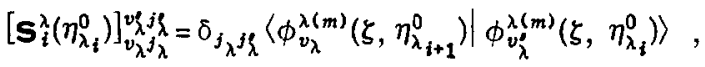

and the transformation between Regions III and IV is achieved with equations

$$
\mathbf{g}_{J}^{\lambda(m) \pm}\left(\eta_{\lambda_{0}} ; \eta_{\lambda_{1}}^{0}\right)=\zeta^{1 / 2} \mathbf{g}_{J}^{\lambda(s) \pm}\left(\varphi_{\lambda_{0}} ; \varphi_{\lambda_{n_{11 I}^{\lambda}}^{0}}^{0}\right)
$$

and

$$
\frac{d \mathbf{g}_{J}^{\lambda(m) \pm}}{d \eta_{\lambda}}\left(\eta_{\lambda_{0}} ; \eta_{\lambda_{1}}^{0}\right)=\boldsymbol{\zeta}^{3 / 2} \frac{d \mathbf{g}_{J}^{\lambda(s) \pm}}{d \varphi_{\lambda}}\left(\varphi_{\lambda_{0}} ; \varphi_{\lambda_{n_{1 \mathrm{I}}}^{\lambda}}^{0}\right)
$$

where

$$
\begin{gathered}
\left(\zeta^{b}\right)_{v_{\lambda} \lambda_{\lambda}}^{v_{\lambda}^{\prime} j_{\lambda}^{\prime}} \\
=\delta_{j_{\lambda} j_{\lambda}^{\prime}}\left\langle\phi_{v_{\lambda}}^{\lambda(m)}\left(\zeta ; \eta_{\lambda_{1}}^{0}\right)\left|\left(\frac{\zeta}{\rho_{\lambda_{0}}-\zeta}\right)^{b}\right| \phi_{v_{\lambda}^{\prime}}^{\lambda(s)}\left(\rho_{\lambda_{0}}-\zeta ; \varphi_{\lambda_{n_{I} \lambda}}^{0}\right)\right\rangle \\
b=\frac{1}{2}, \frac{3}{2} .
\end{gathered}
$$




\section{E. Integration of the Schrödinger equation}

We generate solutions $\mathbf{g}_{J}^{\lambda^{+}}$and their derivatives with respect to the propagation variable by choosing at $R_{\lambda}$ $=R_{\lambda_{0}}^{\prime}$ arbitrary initial values for these two matrices and integrating numerically Eqs. (3.38), (3.47), and (3.60) from the beginning of Region II to the end of Region IV. Similarly, we generate the $\mathbf{g}_{J}^{\lambda-}$ solutions and their derivatives by choosing arbitrary initial values for these two matrices at $\eta_{\lambda}=\eta_{\lambda_{1}}$ and numerically integrating these equations from the end of Region IV to the beginning of Region II.

Any appropriate numerical procedure may be employed to integrate the coupled equations. The one we used, which is well suited to equations of the type of Eqs. (3.38), (3.47), and (3.60), is the one developed by Gordon. ${ }^{35}$ In Region II, it can be applied without modification, ${ }^{36}$ but in Regions III and IV, the nonsymmetric nature of the effective interaction potential matrices [Eqs. (3.48) and (3.61)] requires a short modification of the method. As formulated by Gordon, in propagating a system of coupled equations, a transformation to a representation in which the effective potential matrix (say U) becomes diagonal is required. This necessitates finding the eigenvalues of $U$. In the special case where $\mathbf{U}$ is real and symmetric, these eigenvalues are real and the eigenvector matrix is orthogonal. This simplifies the calculation and allows for an efficient program. The effective potential matrices of Regions III and IV as given by Eqs. (3.48) and (3.61) are real but nonsymmetric and thus could have complex eigenvalues and eigenvectors. These potential matrices are, however, equal to the product of two symmetric matrices, one of which ( $\rho_{\lambda}^{2}$ for-Region III and $\zeta^{2}$ for Region IV) is the matrix representation of a positive definite operator $\left(\rho_{\lambda}^{2}\right.$ or $\zeta^{2}$ ) and hence has positive definite eigenvalues. By using these special properties to define the "square root" of $\rho_{\lambda}^{2}$ and $\zeta^{2}$, it is shown in Appendix B that both the eigenvalues and eigenvectors of the effective potential matrix are always real but the eigenvector matrix is not generally orthogonal. This allows us to use real variables throughout the integration, with the only major program changes from the usual Gordon method being in the routines needed to find the eigenvalues and eigenvectors of the effective potential matrix, and in the manipulation of the eigenvector matrices.

\section{F. Restrictions and limitations}

We should at this point summarize the possible limitations and restrictions on the method other than those inherent in the numerical procedures involved or those arising from computer limitations.

First, the choice of matching surfaces in Eqs. (3.2) is largely determined by the shape of the potential energy surface in Fig. 2, and for many reactions, an efficient choice will require somewhat different matching planes. A change in the exact mathematical form of the matching surfaces can significantly affect the details of the integration in Region IV and in the matching procedure although the basic concepts will be preserved. In choosing the matching surfaces for a given reaction, primary consideration should be given to ob- taining an efficient representation of the wavefunction in the vicinity of the matching surfaces. This requires us to avoid too large a potential anisotropy in the matching region, for in that case, our coupled-channel solution will require many closed rotational channels for convergence. At the same time, too small a potential anisotropy (resulting in considerable flux "leakage" through $\gamma_{\lambda}=\pi / 2$ configurations in the matching region) can result in poor convergence of the matching procedure (as will be discussed in Sec. IV). One therefore needs to be clever in choosing these surfaces, and it is possible that for certain reactions, no choice satisfies all of the above criteria and simultaneously leads to a mathematically tractable matching procedure.

One case where the choice of surfaces specified by Eqs. (3.2) leads to a set of coupled equations in the matching region [Eqs. $(3,60)$ ], which is not easily solved without including a large number of closed rotational channels, is when $\alpha_{\nu \lambda}$ (or $\left.\alpha_{\lambda \kappa}\right) \geq 3 \pi / 4$. [See Eq. (A3) for the definition of these angles.] The reason for this is that when this inequality is satisfied, integration in the matching region will extend into those regions of configuration space for which $r_{\nu}$ (or $r_{R}$ ) $=0$, and hence will sample the very high potential energy in those configurations [for which $\gamma_{\lambda}=0$ (or $\pi$ )] while possibly sampling low potential energy for other configurations (near $\gamma_{\lambda}=\pi / 2$ ). That this is the case can be verified by noting that during the integration in the matching region, $\eta_{\lambda}$ must scan the range from $\left(\pi-\alpha_{\nu \lambda}\right) / 2$ to $\pi / 4$ [from Eqs. (3.14), (3.15), and (3.17)] and that Eq. (A5) yields $r_{\nu}=0$ when $\gamma_{\lambda}=0$ and $\alpha_{\nu \lambda} \geq 3 \pi / 4$ at some $\eta_{\lambda}$ within this range. From Eqs. (A3) we note that $\alpha_{\nu \lambda} \geq 3 \pi / 4$ implies $m_{k}\left(m_{\lambda}\right.$ $\left.+m_{\nu}+m_{k}\right)<m_{\lambda} m_{\nu}$, so that this restriction applies primarily to reactions with one atom of the triatomic system considerably lighter than the other two. We should also point out that this problem can be eliminated by the use of a different coordinate system in the matching region or by the use of "rotationally adiabatic" rotational basis functions [rather than $\varphi_{j_{\lambda}}\left(\gamma_{\lambda}\right)$ ].

When one of the three arrangement channels is closed, the method must be somewhat modified, ${ }^{21}$ but this is not a complication. Other practical limitations to the method occur for reactions where break-up collisions are allowed or for which virtual excitations to closed dissociative channels influence the other reactive and nonreactive processes significantly. The present method is not capable of treating any dissociation process, but this is not a serious limitation for many reactions at energies of chemical interest. A more general method which utilizes generalized hyperspherical functions is currently being developed in these laboratories for the purpose of treating both break-up and rearrangement collisions.

\section{THE MATCHING}

\section{A. Projection of the wavefunction onto the matching surface basis functions}

At the completion of the integration in arrangement channel region $\lambda$, we have generated solutions which satisfy the Schrödinger equation in that region, but which do not, in general, match smoothly with the cor- 
responding wavefunctions obtained from the integrations in the other arrangement channel regions $\nu$ and $\kappa$. In this section we describe the procedure for accomplishing this smooth matching. This procedure may conveniently be broken up into two parts. First, we consider the projection of the full wavefunction and its derivative normal to the matching surface onto a set of orthonormal basis functions which span the matching surfaces. Second, we take linear combinations of the wavefunctions in each arrangement channel region and match them smoothly, on these surfaces, to linear combinations of the corresponding wavefunctions in the other arrangement channel regions. The resulting matrix equations can be solved to yield a set of solutions which satisfy Condition (a) of Sec. II. A and can subsequently (see Sec. V.A) be used to form the scattering solutions satisfying Condition (c) of that section.

Let us consider the projection procedure for the matching surface $\pi_{\nu \lambda}$ [Fig. 2(a)]. The analogous equations for $\pi_{k \nu}$ and $\pi_{\lambda k}$ are obtained from those derived below by cyclic permutation of the indices $\lambda \nu \kappa$. We first consider the wavefunction on $\pi_{\nu \lambda}$ obtained from the integration in arrangement channel $\lambda$. Using Eqs. (3.59), (3.5), (2.13), and $(2.10)$, we get the following expression for the wavefunction in Region IV (Subregion $i$ ):

$$
\begin{aligned}
& \Psi_{j}^{\lambda v}{ }_{\lambda}^{{ }^{\prime} \lambda_{\lambda}^{ \pm}}\left(\theta_{\lambda}, \zeta, \eta_{\lambda}, \gamma_{\lambda}\right)
\end{aligned}
$$

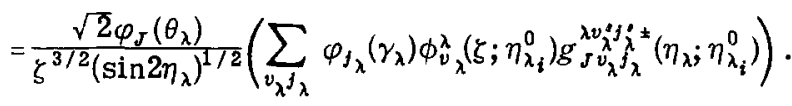

Throughout Sec. IV we will consider superscript $(m)$ of Sec. III. D. 4 to be present implicitly and will omit writing it explicitly. We have, however, added the indices $v_{\lambda}^{\prime} j_{\lambda}^{\prime}$ to denote different linearly independent solutions obtained by using different initial conditions in the numerical solution of the Schrödinger equation. As explained in Sec. III. D. 1, if we truncate the close coupling expansion after $N$ vibration-rotation basis functions, there will be $N$ sets of indices $v_{\lambda}^{\prime} j_{\lambda}^{\prime}$ in Eq. $(4,1)$.

The expression in large parentheses in Eq. (4.1) is expanded in terms of a different set of vibrational functions $\phi_{v_{\lambda}}^{\lambda}\left(\zeta ; \eta_{\lambda_{i}}^{0}\right)$ for each subregion $i$ of Region IV. We now transform to a representation in terms of a single set of vibration-rotation basis functions $\varphi_{j_{\lambda}}\left(\gamma_{\lambda}\right) \phi_{v_{\lambda}}^{\lambda}(\zeta)$ for all of Region IV. The actual functions $\phi_{v_{\lambda}}^{\lambda}(\zeta)$ to be used are arbitrary, but for reactions such as $\mathrm{H}+\mathrm{H}_{2}$ which favor reaction through collinear geometries, the most efficient set for this purpose (as will be explained in more detail below) are the functions $\phi_{v_{\lambda}}^{\lambda}\left(\zeta ; \eta_{\lambda_{0}}\right)$ defined along the cut $\eta_{\lambda}=\eta_{\lambda_{0}}$, and which will be denoted by $\phi_{v_{\lambda}}^{\lambda}(\zeta)$ simply. The transformation equations associated with the change in the vibrational basis set are given by equations similar to Eqs. (3.41) and (3.53), where a different overlap matrix will be generated for each subregion $i$. We will denote the new " $g$ " function thus obtained by $g_{5 v_{\lambda}^{\lambda} \lambda_{\lambda}^{\prime} j_{\lambda}^{*}}\left(\eta_{\lambda}\right)$ (we will omit the parameter $\eta_{\lambda_{0}}$ ). With this change of vibrational basis, we can write a single expression for $\Psi_{J}^{\lambda v_{\lambda}^{\prime} \lambda_{\lambda}^{ \pm}}$valid throughout all of Region IV:

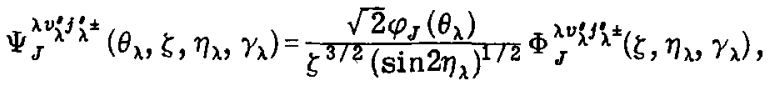

where

$$
\Phi \underset{J}{\lambda v^{j} j \dot{\lambda}^{ \pm}}=\sum_{v_{\lambda} j_{\lambda}} \varphi_{j_{\lambda}}\left(\gamma_{\lambda}\right) \phi_{v_{\lambda}}^{\lambda}(\zeta) g_{J v_{\lambda}^{j} j_{\lambda}}^{\lambda u_{\lambda}^{\prime j} j_{\lambda}^{ \pm}}\left(\eta_{\lambda}\right)
$$

On the matching surface $\pi_{\nu \lambda}, \eta_{\lambda}$ and $\gamma_{\lambda}$ are related to each other by the equation [from Eqs. (A14) and (3.18)]

$\cot 2 \eta_{\lambda}=-\cot \alpha_{\nu \lambda} \cos \gamma_{\lambda}, \quad 0 \leq \gamma_{\lambda} \leq \frac{\pi}{2}$ and $\frac{3 \pi}{2} \leq \gamma_{\lambda}<2 \pi$.

Hence, if we substitute $\eta_{\lambda}\left(\gamma_{\lambda}\right)$ as obtained from Eq. (4.4) into Eqs. (4.2) and (4.3), we obtain the desired wavefunction on $\pi_{\nu \lambda}$ 。

In order to insure a smooth matching of the wavefunctions, we need also to match the derivative of the wavefunction of Eqs. (4.2) and (4.3) at the matching surfaces. Although many types of derivatives are possible, the normal derivative $\partial / \partial n_{\nu \lambda}$ to the matching half-plane possesses many useful mathematical properties (some of which are seen below), and for this reason we will consider it in the following discussion. Expressions for $\partial / \partial n_{\nu \lambda}$ in terms of $\zeta, \eta_{\lambda}$, and $\gamma_{\lambda}$ are derived in Appendix A [Eq. (A18)]. Since these are internal configuration coordinates ( $i_{a} e_{0}$, those on which $V^{\lambda}$ depends) we are free to choose any one external variable (such as $\theta_{\lambda}$ or $\theta_{\nu}$ ) to hold constant during the differentiation process. We must, however, use this same variable in deriving all equations concerning the derivative matching on $\pi_{\nu \lambda}$. Choosing this external variable to be $\theta_{\lambda}$ and introducing Eq. (A18) into Eqs. (4.2) and (4.3), and then using Eq. (4.4) to express everything on the matching surface, we obtain

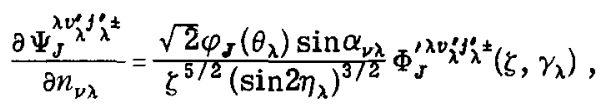

where

$$
\begin{aligned}
& \Phi_{J}^{\prime \prime v \lambda_{\lambda}^{\prime j} \lambda^{ \pm}}\left(\zeta, \gamma_{\lambda}\right) \\
& =\sum_{v_{\lambda} j_{\lambda}} \phi_{v_{\lambda}}^{\lambda}(\zeta) \varphi_{J_{\lambda}}\left(\gamma_{\lambda}\right)\left(\frac{1}{2} \frac{d g_{J}^{\lambda v \nu_{\lambda}^{\prime} j_{\lambda}^{j^{ \pm}}}}{d \eta_{\lambda}}+\cot \alpha_{\nu \lambda}\left(\frac{1}{2} \cos \gamma_{\lambda}\right.\right. \\
& \left.\left.+i j_{\lambda} \sin \gamma_{\lambda}\right) g_{J}^{\lambda \nu_{\lambda}^{\prime j} j_{\lambda}^{*} j_{\lambda}^{*}}\left[\eta_{\lambda}\left(\gamma_{\lambda}\right)\right]\right) .
\end{aligned}
$$

The derivative $d g / d \eta_{\lambda}$ must be evaluated by allowing $\eta_{\lambda}$ to be independent of $\gamma_{\lambda}$ before the expression $\eta_{\lambda}=\eta_{\lambda}\left(\gamma_{\lambda}\right)$ given by $\mathrm{Eq}$. (4.4) is used.

We now wish to expand the functions $\Phi_{J}^{\lambda v \lambda^{j} j_{\lambda^{*}}^{*}}$ and $\Phi_{\partial}^{\prime \lambda v \lambda_{\lambda}^{j} \ell_{\lambda^{*}}}$ on the matching surface $\pi_{\nu \lambda}$ in terms of a set of basis functions $B_{v_{\lambda} j_{\lambda}}^{\nu \lambda}$ which span that surface, $\zeta$ and $\gamma_{\lambda}$ being the independent variables. We choose the $B_{v_{\lambda} j_{\lambda}}^{\nu \lambda}$ to be

$$
B_{v_{\lambda}^{j} j^{j}}^{\nu \lambda}\left(\zeta, \gamma_{\lambda}\right)=\phi_{v_{\lambda}}^{\lambda}(\zeta) A_{j_{\lambda}}^{\nu \lambda}\left(\gamma_{\lambda}\right)
$$

where the $\phi_{v_{\lambda}}^{\lambda}$ 's were defined above [in the paragraph preceding Eq. (4.2)], and the $A_{j_{\lambda}}^{\nu \lambda}\left(\gamma_{\lambda}\right)$ are a set of rotational functions which we shall require to be orthonormal and complete on the domain $0 \leq \gamma_{\lambda} \leq \frac{1}{2} \pi, \frac{1}{2} 3 \pi \leq \gamma_{\lambda}$ $<2 \pi$. For convenience, we will also impose the condition that $A_{J_{\lambda}}^{\nu \lambda}\left(\gamma_{\lambda}\right)$ be real and that 


$$
A_{j_{\lambda}}^{\nu \lambda}\left(\gamma_{\lambda}\right)=(-1)^{j} A_{j_{\lambda}}^{\nu \lambda}\left(2 \pi-\gamma_{\lambda}\right) \text {. }
$$

This is not a significantly restrictive assumption, and will lead to matching equations involving only real quantities.

The crucial characteristic in our choice of the rotational basis functions $A$ is that they be complete for the domain $0 \leq \gamma_{\lambda} \leq \frac{1}{2} \pi, \frac{1}{2} 3 \pi \leq \gamma_{\lambda}<2 \pi$. This contrasts with the 0 to $2 \pi$ interval over which the asymptotic rotational functions $\varphi_{j_{\lambda}}\left(\gamma_{\lambda}\right)$ are complete. What we have actually done is to divide the domain of $\gamma_{\lambda}$ into two equal subdomains: (a) 0 to $\frac{1}{2} \pi$ and $\frac{1}{2} 3 \pi$ to $2 \pi$, on which we choose the $\pi_{v \lambda}$ rotational functions $A_{j_{\lambda}}^{\nu \lambda}\left(\gamma_{\lambda}\right)$, and (b) $\frac{1}{2} \pi$ to $\frac{1}{2} 3 \pi$, where we use the analogous functions $A_{j_{\lambda}}^{\lambda_{k}}\left(\gamma_{\lambda}\right)$ which span $\pi_{\lambda x}$. The union $A_{j_{\lambda}}^{\lambda_{\lambda}}\left(\gamma_{\lambda}\right)$ of the two sets of functions $\left\{A_{j_{\lambda}}^{\nu \lambda}, A_{j_{\lambda}}^{\lambda_{k}}\right\}$ forms a set of rotational functions which spans $(0,2 \pi)$ and this allows us to establish a one-to-one correspondence between the rotational functions $A_{j_{\lambda}}^{\lambda}$ which span the pair of matching surfaces $\pi_{\nu \lambda}$ and $\pi_{\lambda K}$, and the asymptotic functions $\varphi_{j_{\lambda}}^{\lambda}\left(\gamma_{\lambda}\right)$. More specifically, if we have $N$ vibration-rotation basis functions asymptotically in arrangement channel $\lambda$, the sum of the number of $B^{\nu \lambda}$ and $B^{\lambda k}$ functions must be $N$. For a symmetric reaction (i. e., one in which channels $\nu$ and $\kappa$ are equivalent), we must use $N / 2$ functions in each of these two sets. In this case, the rotational quantum numbers $j_{\lambda}$ spanned by the $B_{v_{\lambda} j_{\lambda}}^{\nu \lambda}$ (for each vibrational quantum number $v_{\lambda}$ ) include only one-half of those spanned by the asymptotic functions $\varphi_{j_{\lambda}}$. The number of the latter should in such cases be even, which is not a severe constraint.

We now discuss possible choices for the functions $A_{\xi_{\lambda}}^{\nu \lambda}$. Two sets of functions which are both orthonormal and complete over the range $0 \leq \gamma_{\lambda} \leq \frac{1}{2} \pi$ and $\frac{1}{2} 3 \pi \leq \gamma_{\lambda}<2 \pi$ are $(\pi)^{-1 / 2} \exp \left(2 i j_{\lambda} \gamma_{\lambda}\right)$ and $(\pi)^{-1 / 2} \exp \left[\left(2 j_{\lambda}+1\right) i \gamma_{\lambda}\right]$ for $j_{\lambda}$ $=0, \pm 1, \pm 2, \ldots$ These do not satisfy the condition of Eq. (4.8), but certain linear combinations of them do such as (a) $(\pi)^{-1 / 2},(2 / \pi)^{1 / 2} \cos 2 \gamma_{\lambda},(2 / \pi)^{1 / 2} \sin 2 \gamma_{\lambda}$, $(2 / \pi)^{1 / 2} \cos 4 \gamma_{\lambda},(2 / \pi)^{1 / 2} \sin 4 \gamma_{\lambda}, \ldots$, and $(b)(2 / \pi)^{1 / 2} \sin \gamma_{\lambda}$, $(2 / \pi)^{1 / 2} \cos \gamma_{\lambda},(2 / \pi)^{1 / 2} \sin 3 \gamma_{\lambda},(2 / \pi)^{1 / 2} \cos 3 \gamma_{\lambda}, \ldots$ We can also choose mixtures of (a) and (b) above such as (c) $(2 / \pi)^{1 / 2} \cos \gamma_{\lambda},(2 / \pi)^{1 / 2} \sin 2 \gamma_{\lambda},(2 / \pi)^{1 / 2} \cos 3 \gamma_{\lambda}, \ldots$ This last set of functions (corresponding to $j_{\lambda}=0,1$, $2, \ldots$, respectively) has the property that all its members vanish at $\gamma_{\lambda}=\pi / 2$ and $3 \pi / 2$. This makes these functions very efficient for expanding the wavefunctions for certain reactions, as described below. An alternative to analytical functions would be a numerically determined set such as the rotational eigenfunctions for some approximate potential on the matching surface. To see what these functions might look like for $\mathrm{H}+\mathrm{H}_{2}$, in Fig. 6 we plot contours of the Porter-Karplus potential $^{31}$ on the two-dimensional matching half-plane $\pi_{\nu \lambda}$. The lines converging at the origin are intersections of $\pi_{\nu \lambda}$ with $\gamma_{\lambda}=$ constant planes and correspond therefore to constant values of $\eta_{\lambda}$. The figure indicates that only the region of the matching surface for which $\gamma_{\lambda}$ is in the range $0-60^{\circ}$ and $300^{\circ}-360^{\circ}$ (and $\eta_{\lambda}$ in the range $\eta_{\lambda_{0}}=30^{\circ}-36.9^{\circ}$ ) has a low enough potential energy to contribute significantly to the reaction at energies less than $1 \mathrm{eV}$. This implies that we should choose a basis set $B^{\nu \lambda}$ which describes the wavefunction best near

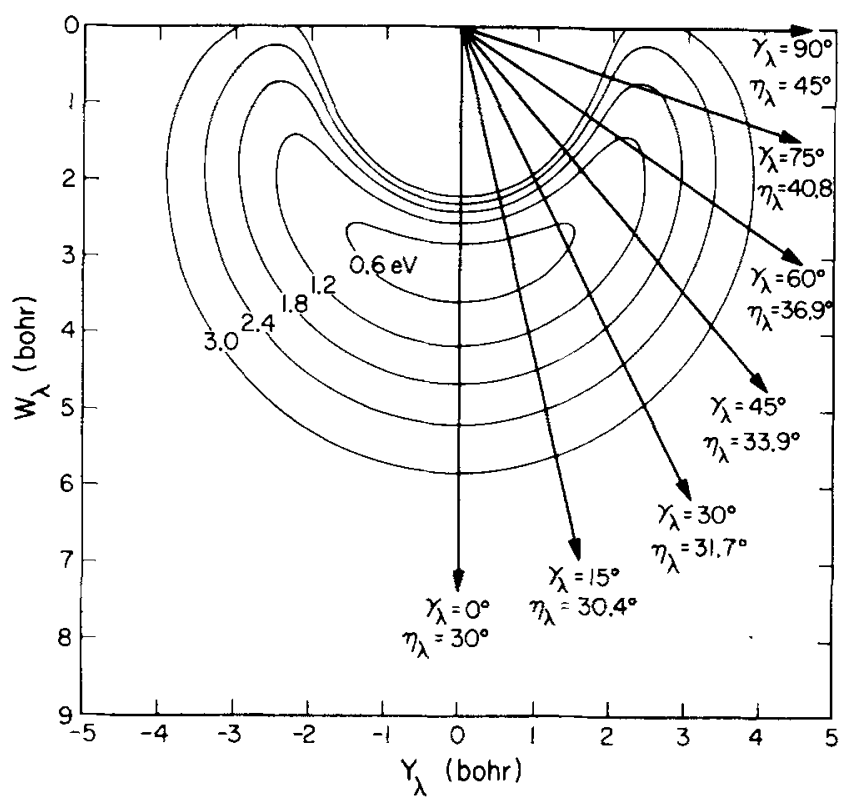

FIG. 6. Plot of equipotential contours on the matching surface $\pi_{\nu \lambda}$ for the $\mathrm{H}+\mathrm{H}_{2}$ reaction in the Cartesian coordinate system $W_{\lambda}, Y_{\lambda}$, where $W_{\lambda}=\left(X_{\lambda}^{2} \sin ^{2} \alpha_{\nu \lambda}+Z_{\lambda}^{2} \cos ^{2} \alpha_{\nu \lambda}\right)^{1 / 2}$ and the system $X_{\lambda}, Y_{\lambda}, Z_{\lambda}$ is the one described in Sec. III.A. Because of the symmetry of the $\mathrm{H}_{3}$ system, Fig. 6 is equivalent to the lower half of Fig. 2(c) since the $Z_{\lambda}<0$ half-plane of the $O Y_{\lambda} Z_{\lambda}$ plane is for this system the same as the $\pi_{k \nu}$ matching plane. The half-lines emanating from the origin correspond to constant values of $\gamma_{\lambda}$ and $\eta_{\lambda}$, as indicated. To each such half-line, for which $\gamma_{\lambda}=\bar{\gamma}_{\lambda}$, there corresponds a symmetric one with respect to the vertical axis, having the same value of $\eta_{\lambda}$ and a value of $\gamma_{\lambda}=360^{\circ}-\bar{\gamma}_{\lambda}$ in the $270^{\circ}$ to $360^{\circ}$ range.

$\gamma_{\lambda}=0$, i. e., near $\eta_{\lambda}=\eta_{\lambda_{0}}$. For this reason, we previously chose the vibrational eigenfunctions $\phi_{v_{\lambda}}^{\lambda}(\zeta)$ to be solutions to Eq. (3.5) for $\eta_{\lambda}=\eta_{\lambda_{0} \cdot}$ ．See paragraph preceding Eq. (4.2)]. Although there is no single $\gamma_{\lambda}$-dependent rotational potential which describes the rotational motion on the matching surface correctly for all $\zeta$, it should be clear from Fig. 6 that rotational functions which are numerically determined from the potential on the matching surface for a fixed $\zeta$ (such as the $\zeta$ for which this potential, at $\gamma_{\lambda}=0$, has a minimum) will be localized near $\gamma_{\lambda}=0$ and must be close to zero near $\gamma_{\lambda}$ $=\pi / 2$ and $3 \pi / 2$. For this reason, the analytical set (c) should be efficient for expanding the wavefunction on $\pi_{\nu \lambda}$. Finally, we should mention that an important test of the correctness of the method is to demonstrate the invariance of the final converged results with respect to the choice of $A_{j_{\lambda}}^{\lambda}$.

We now expand the functions $\Phi_{J}^{\lambda v_{\lambda}^{\prime} j j_{\lambda}^{*}}$ and $\Phi_{j}^{\prime \lambda v_{\lambda}^{\prime} j_{\lambda}^{*}}[\mathrm{de}-$ fined by Eqs. (4.3) and (4.6)] in terms of the matching surface functions $B_{v_{\lambda} j_{\lambda}}^{\nu \lambda}$ as follows:

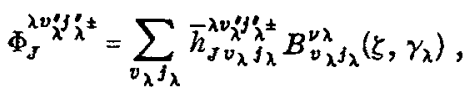

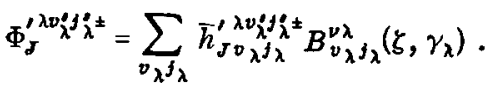

Whereas the indices $v_{\lambda}^{\prime} j_{\lambda}^{\prime}$ assume $N$ values, $v_{\lambda} j_{\lambda}$ assume fewer values than that. In view of our previous discussion, $N / 2$ is a convenient choice for this number for 
the highly symmetric $\mathrm{H}_{3}$ system. The coefficients

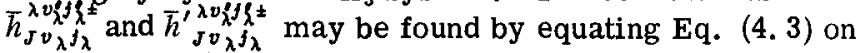
$\pi_{\nu \lambda}$ with Eq. (4.9), and Eq. (4.6) with Eq. (4.10), multiplying the resulting expressions by $B_{v_{\lambda}^{\prime \prime j}, j^{\prime \prime}}^{* \nu \lambda}$ and integrating them over $\zeta$ and $\gamma_{\lambda}$. Using the orthonormality of the $B_{{ }^{\nu}{ }^{j} \lambda}^{\nu \lambda}$ functions on the matching surface and Eq. (4.8), and interchanging in the end the $v_{\lambda}^{\prime \prime} j_{\lambda}^{\prime \prime}$ indices with the $v_{\lambda} j_{\lambda}$ ones, we get

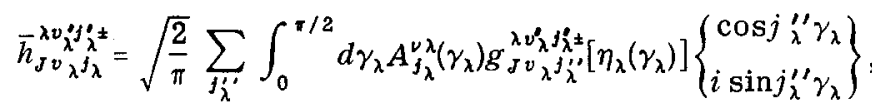

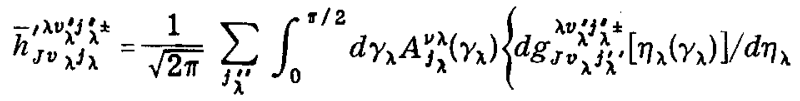

$$
\begin{aligned}
& \times\left(\begin{array}{c}
\cos j_{\lambda}^{\prime \prime} \gamma_{\lambda} \\
i \sin j_{\lambda}^{\prime \prime} \gamma_{\lambda}
\end{array}\right)+\cot \alpha_{\nu \lambda}\left[\left(j_{\lambda}^{\prime \prime}+\frac{1}{2}\right)\left(\begin{array}{c}
\cos \left(j_{\lambda}^{\prime \prime}+1\right) \gamma_{\lambda} \\
i \sin \left(j_{\lambda}^{\prime \prime}+1\right) \gamma_{\lambda}
\end{array}\right)\right. \\
& \left.-\left(j_{\lambda}^{\prime \prime}-\frac{1}{2}\right)\left(\begin{array}{c}
\cos \left(j_{\lambda}^{\prime \prime}-1\right) \gamma_{\lambda} \\
i \sin \left(j_{\lambda}^{\prime \prime}-1\right) \gamma_{\lambda}
\end{array}\right) g_{J v_{\lambda}^{j} v_{\lambda}^{\prime \prime}}^{\lambda v_{\lambda}^{\prime j} \dot{\lambda}^{ \pm}}\left[\eta_{\lambda}\left(\gamma_{\lambda}\right)\right]\right\},
\end{aligned}
$$

where the upper term in the large parentheses is to be used for even $j_{\lambda}$, and the lower for odd $j_{\lambda}$, and use was made of Eq. (4.8).

We now must consider the expansion of the wavefunction obtained from the integration in channel $\nu$, in the same matching surface basis functions $B_{v_{\lambda^{j} \lambda}}^{\nu_{\lambda}}$ of Eq.

(4. 7). To do this we first express the wavefunction and its normal derivative in a form analogous to Eqs. (4.2), (4. 3), (4.5), and (4.6). For the wavefunction we get

$$
\Psi_{J}^{\nu \nu_{\nu}^{\prime} j_{\nu}^{ \pm}}=\frac{\sqrt{2} \varphi_{J}\left(\theta_{\nu}\right)}{\zeta^{3 / 2}\left(\sin 2 \eta_{\nu}\right)^{1 / 2}} \Phi_{J}^{\nu v_{\nu}^{\prime} f_{\nu}^{\prime}}\left(\zeta, \eta_{\nu}, \gamma_{\nu}\right)
$$

where

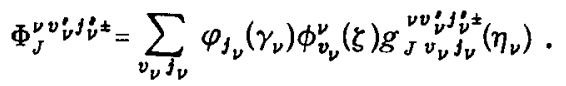

The vibrational basis function $\phi_{v_{\nu}}^{\nu}(\zeta)$ is defined in a manner similar to $\phi_{v_{\lambda}}^{\lambda}(\zeta)$ and, in fact, may be identical to it (for $v_{\nu}=v_{\lambda}$ ) if the reference potentials are defined appropriately. In terms of the $v$ arrangement channel coordinates, the relation between $\eta_{\nu}$ and $\gamma_{\nu}$ on the matching surface $\pi_{\nu \lambda}$ is given by

$$
\cot 2 \eta_{\nu}=\cot \alpha_{\nu \lambda} \cos \gamma_{\nu} \quad \frac{1}{2} \pi \leq \gamma_{\nu} \leq \frac{1}{2} 3 \pi .
$$

This is easily derived from Eq. (4.4), using the relations (A12) and (A15) of the Appendix and Eq. (3.18).

In calculating the normal derivative of the wavefunc- tion of Eq. (4.13) on the matching surface $\pi_{\nu \lambda}$, we must remember that $\theta_{\lambda}$ must be held constant during the differentiation. It is therefore desirable to express $\theta_{\nu}$ in terms of $\theta_{\lambda}$,

$$
\theta_{\nu}=\theta_{\lambda}+\Delta_{\nu \lambda},
$$

where $\Delta_{\nu \lambda}$ is a function of the internal variables $R_{\lambda}, r_{\lambda}$, $\gamma_{\lambda}$ only as seen in Eqs. (A9) of the Appendix. If we express those two equations in terms of the variables $\zeta$, $\eta_{\lambda}$, and $\gamma_{\lambda}$, then find the normal derivative of $\Delta_{\nu \lambda}$ using Eq. (A18) and finally use Eq. (4.4) to express all quantities on the matching surface $\pi_{\nu \lambda}$, we obtain the very useful equation valid only on this surface:

$$
\frac{\partial \Delta_{\nu \lambda}}{\partial n_{\nu \lambda}}\left(\zeta, \eta_{\lambda}, \gamma_{\lambda}\right)=0
$$

As a consequence of this simplification, we can express the normal derivative of the wavefunction in arrangement channel $\nu$ on $\pi_{\nu \lambda}$ by

$$
\frac{\partial \Psi_{J}^{\nu \nu_{\nu}^{j} i_{\nu}^{ \pm}}}{\partial n_{\nu \lambda}}=\frac{\sqrt{2} \varphi_{J}\left(\theta_{\lambda}\right) e^{i J \Delta \nu \lambda}}{\zeta^{5 / 2}\left(\sin 2 \eta_{\nu}\right)^{3 / 2}} \Phi_{J}^{\prime \nu v_{\nu}^{j} \nu_{\nu}^{ \pm}}\left(\zeta, \gamma_{\nu}\right)
$$

where

$$
\begin{aligned}
\Phi_{J}^{\prime \nu v_{\nu}^{\prime} j_{\nu}^{ \pm}}\left(\zeta, \gamma_{\nu}\right)= & \sum_{j_{\nu} v_{\nu}} \varphi_{j_{\nu}}\left(\gamma_{\nu}\right) \phi_{v_{\nu}}^{\nu}(\zeta)\left[\frac{1}{2}\left(\frac{d g_{J v_{\nu}^{j}}^{\nu v_{\nu}^{j} j_{\nu}^{ \pm}}}{d \eta_{\nu}}\right)_{\eta_{\nu}^{ \pm \eta_{\nu}}\left(\gamma_{\nu}\right)}\right. \\
& \left.-\cot \alpha_{\nu \lambda}\left(\frac{1}{2} \cos \gamma_{\nu}+i j_{\nu} \sin \gamma_{\nu}\right) g_{J}^{\nu v_{\nu}^{\prime} j_{\nu}^{\prime} \nu_{\nu}^{ \pm}}\left[\eta_{\nu}\left(\gamma_{\nu}\right)\right]\right] .
\end{aligned}
$$

We now expand the functions $\Phi_{J}^{\nu v_{\nu} f_{\nu^{ \pm}}}$and $\Phi_{J}^{\nu v_{\nu} f_{\nu \pm}^{ \pm}}$in terms of the matching surface functions $B_{v_{\nu} f_{\nu}}^{\nu \lambda}\left(\zeta, \gamma_{\lambda}\right)$. Note that we use the $\lambda$ arrangement channel variables to express the $\nu$ arrangement channel wavefunction, using the transformation equations (A12), (A14), and (3.18) to relate the $\lambda$ and $\nu$ sets of variables. The resulting expansions are

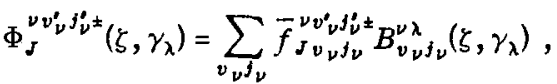

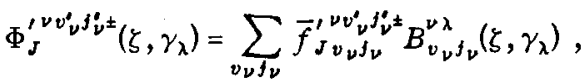

where the expansion coefficients are given by

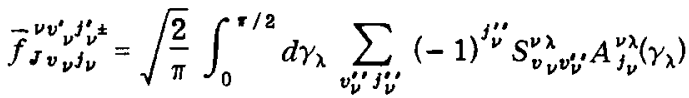

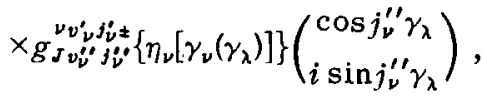

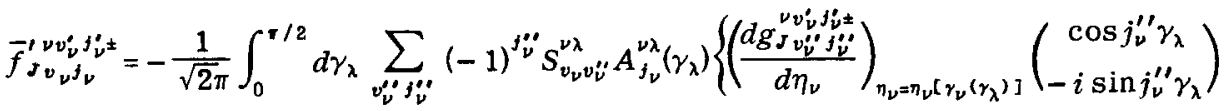

$$
\begin{aligned}
& \left.+\cot \alpha_{\nu \lambda}\left[\left(j_{\nu}^{\prime \prime}-\frac{1}{2}\right)\left(\begin{array}{c}
\cos \left(j_{\nu}^{\prime \prime}+1\right) \gamma_{\lambda} \\
-i \sin \left(j_{\nu}^{\prime \prime}+1\right) \gamma_{\lambda}
\end{array}\right)-\left(j_{\nu}^{\prime \prime}-\frac{1}{2}\right)\left(\begin{array}{c}
\cos \left(j_{\nu}^{\prime \prime}-1\right) \gamma_{\lambda} \\
-i \sin \left(j_{\nu}^{\prime \prime}-1\right) \gamma_{\lambda}
\end{array}\right)\right] g_{J \nu_{\nu}^{\prime \prime} v_{\nu}^{\prime \prime}}^{v_{\nu}^{\prime \prime}}\left\{\eta_{\nu}\left[\gamma_{\nu}\left(\gamma_{\lambda}\right)\right]\right\}\right\},
\end{aligned}
$$


where

$$
S_{v_{\nu} v_{\nu^{\prime}}}^{\nu \lambda}=\int_{0}^{\infty} \phi_{v_{\nu}}^{\lambda}(\zeta) \phi_{v_{\nu^{\prime}}}^{\nu}(\zeta) d \zeta
$$

As before, the upper term in the large parentheses is used for even $j_{v}$ and the lower for odd $j_{v}$.

\section{B. The matching equations}

With the wavefunctions from the integrations in both channels $\nu$ and $\lambda$ expressed in terms of the basis set $B_{v_{\lambda} j_{\lambda}}^{\nu \lambda}\left(\zeta, \gamma_{\lambda}\right)$ on $\pi_{\nu \lambda}$, we can now take the appropriate linear combinations of these solutions to yield solutions to the full Schrödinger equation which are continuous and smooth throughout all of configuration space. Let us denote the fully matched solutions thus obtained by $\Psi_{J}^{(i) \cup j}$. There are $N$ sets of indices $v j$, and the superscript $(i)$ can have the values 1,2 , or 3 . The full set of indices $(i) v j$ thus scans $3 N$ values, and we will therefore obtain $3 N$ linearly independent solutions to the Schrödinger equation. ${ }^{37}$

We now write $\Psi_{J}^{(i) v j}$ in terms of the solutions $\Psi_{J}^{\lambda v_{\lambda} j_{\lambda}^{ \pm}}$ and also in terms of the $\Psi_{J}^{\nu v_{\nu} \nu^{ \pm}}$:

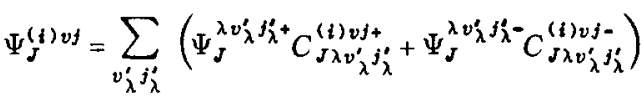

$$
\begin{aligned}
& =\sum_{\nu_{\nu}^{\prime} j_{\nu}^{j}}\left(\Psi_{J}^{\nu v_{\nu}^{\prime} j_{\nu}^{+}} C_{J \nu v_{\nu}^{j} j_{\nu}^{j}}^{(i) v j+}+\Psi_{J}^{\nu v_{\nu}^{i} j i^{-}-} C_{J \nu v_{\nu}^{\prime} j_{\nu}^{j}}^{(i) v j-}\right)
\end{aligned}
$$

and

$$
\begin{aligned}
& \frac{\partial \Psi_{J}^{(i) v j}}{\partial n_{\nu \lambda}}=\sum_{v_{\lambda}^{\prime} j_{\lambda}^{\prime}}\left(\frac{\partial \Psi_{J}^{\lambda v_{\lambda}^{\prime} j_{\lambda}^{+}}}{\partial n_{\nu \lambda}} C_{J \lambda v_{\lambda}^{\prime} j_{\lambda}^{\prime}}^{(i) v j^{+}}+\frac{\partial \Psi_{J}^{\lambda v_{\lambda}^{\prime} j_{\lambda}^{\prime}}}{\partial n_{\nu \lambda}} C_{J \nu v_{\nu}^{\prime} j_{\nu}^{j}}^{(i) v j}\right)
\end{aligned}
$$

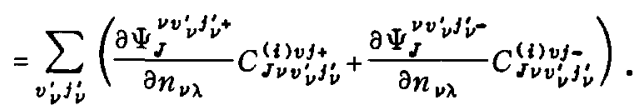

The coefficients $C_{J_{\lambda} v_{\lambda}^{j} j_{\lambda}^{j}}^{(i) v j^{ \pm}}$and $C_{J_{\nu} v_{\nu} j_{\nu}}^{(i) j_{j}}$ are to be determined by applying these two sets of equations on $\pi_{\nu \lambda}$ (and similar equations on $\pi_{k \nu}$ and $\pi_{\lambda_{k}}$ ), and by imposing the scattering solution boundary conditions as explained in Sec. V.A. Equations $(4.25)$ and $(4.26)$, when evaluated on $\pi_{\nu \lambda}$, comprise the smooth matching conditions.

By substituting Eqs. (4.2), (4.13), and (4.16) into Eqs. (4.25), we obtain the following expression:

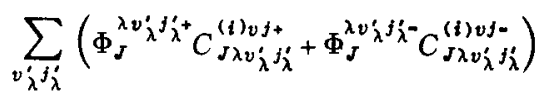

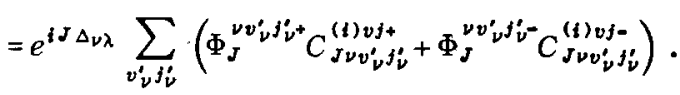

The analogous expression for the normal derivatives is obtained from Eq. (4.27) by replacing the $\Phi$ by the $\Phi^{\prime}$ in that equation. If we now substitute the expansions given by Eqs. (4.9), (4.10), (4.20), and (4.21) into Eq. (4.27) and into its equivalent for the normal derivatives, then multiply through by $B_{v \lambda j_{\lambda}}^{\nu \lambda}\left(\zeta, \gamma_{\lambda}\right)$, and integrate over $\zeta$ and $\gamma_{\lambda}$, we obtain the following system of linear algebraic equations:

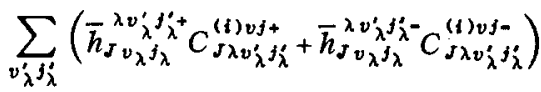

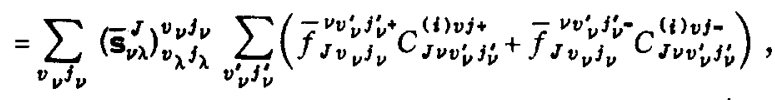

with the analogous equation for derivatives obtained by using $\bar{h}^{\prime}$ and $\bar{f}^{\prime}$ instead of $\bar{h}$ and $\bar{f}$ above. The matrix $\overline{\mathbf{s}}_{\nu \lambda}^{J}$ is defined by

$$
\left(\bar{s}_{\nu \lambda}^{J}\right)_{v_{\lambda} j_{\lambda}}^{v_{\nu} j_{\nu}}=\delta_{v_{\lambda} v_{\nu}}\left\{\begin{array}{l}
2 \int_{0}^{\pi / 2} A_{j_{\lambda}}^{\nu \lambda} A_{j_{\nu}}^{\nu \lambda} \cos J \Delta_{\nu \lambda} d \gamma_{\lambda} \text { for } j_{\lambda}+j_{\nu}=\text { even } \\
2 i \int_{0}^{r / 2} A_{j_{\lambda}}^{\nu \lambda} A_{j_{\nu}}^{\nu \lambda} \sin J \Delta_{\nu \lambda} d \gamma_{\lambda} \text { for } j_{\lambda}+j_{\nu}=\text { odd },
\end{array}\right.
$$

or, by the expression (which is only equivalent to it for a complete set of functions $B^{\nu \lambda}$ )

$$
\bar{s}_{\nu \lambda}^{J}=\exp \left(i J \Delta^{\nu \lambda}\right)
$$

where

$$
\left(\Delta^{\nu \lambda}\right)_{v_{\lambda} f_{\lambda}}^{v_{\nu} f_{\nu}}=\left\langle B_{v_{\lambda} j_{\lambda}}^{\nu_{\lambda}}\left|\Delta_{\nu \lambda}\right| B_{v_{\nu} j_{\nu}}^{\nu \lambda}\right\rangle
$$

As should be evident from Eqs. (4.30) and (4.31), the ma$\operatorname{trix} \overline{\mathbf{s}}_{\nu \lambda}^{J}$ is unitary since $\Delta_{\nu \lambda}$ is real, and therefore $\Delta^{\nu \lambda}$ is Hermitian.

By examining the definitions given in Eqs. (4.11), (4.12), (4.22), (4.23), and (4.29) for the various symbols used in Eq. (4.28) and its equivalent for the derivatives, we find that the latter set of equations involve either purely real terms or purely imaginary terms. Let us omit the factor $i$ appearing in Eqs. (4.11), (4.12), (4.22), and (4.23) and replace it in Eq. (4.29) by $(-1)^{f_{\nu}}$. We denote all of the real coefficients thus obtained by removing the bars which previously appeared above their symbols. Equation (4.28) and its normal derivative counterpart continue to be valid for the unbarred quantities, which permits all of the calculations associated with the matching to be performed using real number arithmetic, a considerable computational simplification. Regarding the real coefficients appearing in these equations as elements of matrices, we can rewrite them as

$$
h_{J}^{\lambda+} C_{J \lambda}^{(i)+}+h_{J}^{\lambda-} C_{J \lambda}^{(i)-}=s_{\nu \lambda}^{J}\left\{f_{J}^{\nu+} C_{J \nu}^{(i)+}+f_{J}^{\nu-} C_{J \nu}^{(i)-}\right\}
$$

and

$$
\mathbf{h}_{J}^{\prime \lambda+} \mathbf{C}_{J \lambda}^{(i)+}+\mathbf{h}_{J}^{\prime \lambda-} \mathbf{C}_{J \lambda}^{(i)-}=\mathbf{s}_{\nu \lambda}^{J}\left(f_{J}^{\prime \nu+} \mathbf{C}_{J \nu}^{(i)+}+\mathbf{f}_{J}^{\prime \nu-} \mathbf{C}_{J \nu}^{(i)-}\right) .
$$

The matrix $\mathbf{s}_{\nu \lambda}^{J}$ is related to $\overline{\mathbf{s}}_{\nu \lambda}^{J}$ by the unitary transformation $\overline{\mathbf{T}}^{\dagger} \overline{\mathbf{s}}_{\nu}^{J} \overline{\mathbf{T}}$, where $\overline{\mathbf{T}}$ is the unitary diagonal matrix whose diagonal elements alternate betweon 1 and $i$. Therefore, if the $B^{\nu \lambda}$ form a complete set, $\mathbf{s}_{\nu \lambda}^{J}$ is unitary, and since it is real it is also orthogonal. As shown in the previous section, the number of basis functions $B_{v_{\lambda} \gamma_{\lambda}}^{\nu \lambda}$ used to expand the wavefunction on $\pi_{\nu \lambda}$ is $N / 2$, where $N$ is the total number of vibration-rotation functions used in the coupled-channel expansion. This implies that there should only be $N / 2$ rows in the matrices $\boldsymbol{h}_{J}^{\lambda t}$. There are, however, $N$ columns because the different columns denote the $N$ linearly independent solutions propagated in either the forward $(+)$ or backwards $(-)$ integrations. Summarizing, the dimensions of the various matrices in Eqs. (4.32) or (4.33) are symbolically represented as follows: 


$$
\begin{aligned}
& (N / 2 \times N)(N \times N)+(N / 2 \times N)(N \times N) \\
& \quad=(N / 2 \times N / 2)[(N / 2 \times N)(N \times N)+(N / 2 \times N)(N \times N)] .
\end{aligned}
$$

We can combine Eqs. (4.32) and (4.33) into a single expression which contains only square $N \times N$ matrices by defining the argumented $N \times N$ matrices $\hat{\mathbf{h}}_{J}^{\lambda \pm}, \hat{\mathbf{f}}_{J}^{\nu \pm}$, and $\hat{\mathbf{s}}_{\nu \lambda}^{J}$ as

$$
\begin{aligned}
& \hat{\boldsymbol{h}}_{J}^{\lambda \pm}=\left(\begin{array}{c}
\mathbf{h}_{J}^{\lambda \pm} \\
\mathbf{h}_{J}^{\lambda \pm}
\end{array}\right), \\
& \hat{\mathbf{f}}_{J}^{\nu \pm}=\left(\begin{array}{c}
\mathbf{f}_{J}^{\nu \pm} \\
\mathbf{f}_{J}^{\prime \nu \pm}
\end{array}\right), \\
& \hat{\mathbf{s}}_{\nu \lambda}^{J}=\left(\begin{array}{cc}
\mathbf{s}_{\nu \lambda}^{J} & 0 \\
0 & \mathbf{s}_{\nu \lambda}^{J}
\end{array}\right),
\end{aligned}
$$

where 0 denotes an $(N / 2 \times N / 2)$ matrix of zeros. The resulting matching equation on $\pi_{\nu \lambda}$ becomes

$$
\hat{\mathbf{h}}_{J}^{\lambda+} \mathbf{C}_{J \lambda}^{(i)+}+\hat{\mathbf{h}}_{J}^{\lambda-} \mathbf{C}_{J \lambda}^{(i)-}=\hat{\mathbf{s}}_{\nu \lambda}^{J}\left(\hat{\mathbf{f}}_{J}^{\nu+} \mathbf{C}_{J \nu}^{(i)+}+\hat{\mathbf{f}}_{J}^{\nu-} \mathbf{C}_{J \nu}^{(i)-}\right)
$$

In order to solve for the unknown coefficient matrices $\mathbf{C}_{J \lambda}^{(i) \pm}, \mathbf{C}_{J \nu}^{(i) \pm}$ (and $\mathbf{C}_{J k}^{(i) \pm}$ ), we must couple Eq. (4.38) with the corresponding equations obtained from the matching on $\pi_{\kappa \nu}$ and $\pi_{\lambda \kappa}$. Using the same notation as in Eq. (4.38), the matching equations on these two additional surfaces are

$$
\hat{h}_{J}^{\nu+} C_{J \nu}^{(i)+}+\hat{h}_{J}^{\nu-} C_{J \nu}^{(i)-}=\hat{\mathbf{s}}_{k \nu}^{J}\left(\hat{f}_{J}^{k+} C_{J K}^{(i)+}+\hat{\mathbf{f}}_{J}^{k-} C_{J K}^{(i)-}\right)
$$

and

$$
\hat{h}_{J}^{K+} C_{J K}^{(i)+}+\hat{h}_{J}^{\kappa-} C_{J K}^{(i)-}=\hat{S}_{\lambda K}^{J}\left(\hat{f}_{J}^{\lambda+} C_{J \lambda}^{(i)+}+\hat{f}_{J}^{\lambda-} C_{J \lambda}^{(i)-}\right)
$$

We can now combine Eqs. (4.38), (4.39), and (4.40) into the following single matrix equation which involves square matrices of dimension $3 N \times 3 N$ :

$$
\mathbf{N}_{J}^{+} \mathbf{C}_{J}^{+}+\mathbf{N}_{J}^{-} \mathbf{C}_{J}^{-}=\mathbf{0}
$$

where

$$
\begin{aligned}
& \mathbf{N}_{J}^{ \pm}=\left(\begin{array}{ccc}
\hat{\mathbf{h}}_{J}^{\lambda \pm} & -\hat{\mathbf{s}}_{\nu \lambda}^{J} \hat{\mathbf{f}}_{J}^{\nu \pm} & 0 \\
0 & \hat{\mathbf{h}}_{J}^{\nu \pm} & -\hat{\mathbf{s}}_{\kappa \nu}^{J} \hat{\mathbf{f}}_{J}^{K \pm} \\
-\hat{\mathbf{s}}_{\lambda k}^{J} \hat{\mathbf{f}}_{J}^{\lambda \pm} & 0 & \hat{\mathbf{h}}_{J}^{K \pm}
\end{array}\right), \\
& \mathbf{C}_{J}^{ \pm}=\left(\begin{array}{lll}
\mathbf{C}_{J \lambda}^{(1) \pm} & \mathbf{C}_{J \lambda}^{(2) \pm} & \mathbf{C}_{J \lambda}^{(3) \pm} \\
\mathbf{C}_{J \nu}^{(1) \pm} & \mathbf{C}_{J \nu}^{(2) \pm} & \mathbf{C}_{J \nu}^{(3) \pm} \\
C_{J K}^{(1) \pm} & \mathbf{C}_{J K}^{(2) \pm} & \mathbf{C}_{J K}^{(3) \pm}
\end{array}\right)
\end{aligned}
$$

and the 0 stands for a matrix of zeros of the appropriate dimensionality. Let us rearrange Eq. (4.41) to the form

$$
\mathbf{C}_{J}^{+}\left(\mathbf{C}_{J}^{-}\right)^{-1}=-\left(\mathbf{N}_{\mathcal{J}}^{+}\right)^{-1} \mathbf{N}_{j}^{-} .
$$

Equation (4.44) is the essential result of the smooth matching procedure. It expresses the unknown coefficients $\mathbf{C}_{J}^{*}$ in terms of the known $\mathbf{N}_{J}^{ \pm}$and thus determines which linear combination of the solutions obtained from the integrations in each arrangement channel region will produce smoothly matched wavefunctions. Of course, Eq. (4.44) supplies only one $3 N \times 3 N$ matrix equation for the two $3 N \times 3 N$ unknown matrices $\mathbf{C}_{J}^{ \pm}$. This tells us that our matched solutions are not completely unique, which is not unexpected since we have not yet specified the asymptotic conditions which our matched wavefunctions must satisfy. We will do so in the next section, and when these additional conditions are combined with Eq. (4.44), we will obtain unique expressions for the coefficient matrices $\mathbf{C}_{s}^{ \pm}$.

\section{ASYMPTOTIC ANALYSIS}

\section{A. The reactance and scattering matrices}

We will now describe how to obtain the reactance $\left(\boldsymbol{R}_{J}\right)$ and scattering $\left(\mathbf{S}_{J}\right)$ matrices from the asymptotic values of the primitive (but smoothly matched) solutions $\Psi_{J}^{(i) \text { of }}$ described in the previous section. These functions are defined in the entire configuration space. Their asymptotic behavior in each of the three arrangement channels $\lambda=\alpha, \beta, \gamma$ can be obtained from Eqs. $(3.22),(3.5),(2.13),(2.10)$, and (4.25) and is

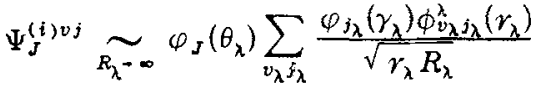

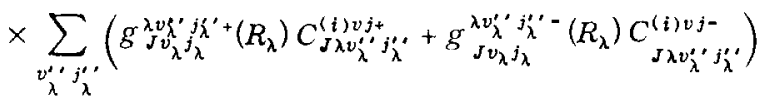

$$
\begin{aligned}
& \lambda=\alpha, \beta, \gamma,
\end{aligned}
$$

where we have dropped the superscript (a) as it will be implicit throughout Sec. V. The product functions $r_{\lambda}^{-1 / 2} \varphi_{j_{\lambda}}\left(\gamma_{\lambda}\right) \phi_{v_{\lambda} j_{\lambda}}^{\lambda}\left(r_{\lambda}\right)$ are the asymptotic vibration-rotation wavefunctions of the diatomic molecule corresponding to the $\lambda$ arrangement channel. We note that $\phi_{\nu_{\lambda} j_{\lambda}}^{\lambda}\left(r_{\lambda}\right)$ vanishes in the asymptotic regions of arrangement channels $\nu$ and $\kappa$ because $r_{\lambda} \rightarrow \infty$ in these regions. As a result, Eq. (5.1) can be rewritten as

$\Psi_{J}^{(i) v j} \sim \sum_{\lambda} \varphi_{J}\left(\theta_{\lambda}\right) \sum_{v_{\lambda} j_{\lambda}} \frac{\varphi_{j_{\lambda}}\left(\gamma_{\lambda}\right) \phi_{v \lambda j_{\lambda}}^{\lambda}\left(r_{\lambda}\right)}{\sqrt{r_{\lambda}} R_{\lambda}} e_{J \lambda v_{\lambda} j_{\lambda}}^{(i) v j}\left(R_{\lambda}\right)$,

where

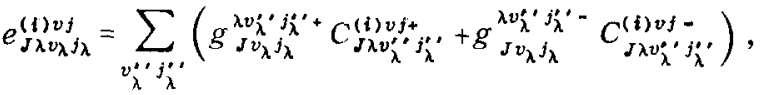

and the $\lambda$ summation extends over arrangement channels $\alpha, \beta$, and $\gamma$. The analogous expression for the function $R_{\lambda}^{-1 / 2}\left(\partial / \partial R_{\lambda}\right)\left(R_{\lambda}^{1 / 2} \Psi_{J}^{(i) v j}\right)$ is obtained from Eqs. (5.2) and (5.3) by replacing $g_{J \nu_{\lambda} j_{\lambda}^{\prime \prime} j_{\lambda}^{\prime \prime}}$ by $d g_{J v_{\lambda} j_{\lambda}}^{\lambda v_{\lambda^{\prime}}^{\prime} j^{\prime \prime}} / d R_{\lambda}$ in the latter.

We now define the reactance and scattering matrix solutions for each partial wave $J$. In analogy with their corresponding three-dimensional multichannel definitions, ${ }^{38,39}$ and using the asymptotic behavior of the coplanar solutions given in Eqs. (3.26), we define the reactance and scattering solutions and the corresponding matrices as follows:

$$
\begin{aligned}
& \Psi_{J}^{\lambda^{\prime} v \lambda^{\prime} \cdot \delta_{\lambda^{\prime}}}[R \text { or } S] \sim \sum_{\lambda} \varphi_{J}\left(\theta_{\lambda}\right) \\
& \sum_{v_{\lambda} j_{\lambda}} \frac{\varphi_{j}^{j}\left(\gamma_{\lambda}\right) \phi v_{\lambda}^{\lambda} \lambda_{\lambda}\left(r_{\lambda}\right)}{\sqrt{r_{\lambda} R_{\lambda}}} b_{J \lambda v_{\lambda} j_{\lambda}}^{\lambda^{\prime} v_{\lambda^{\prime}} j_{\lambda^{\prime}}}[R \text { or } s],
\end{aligned}
$$

where, for the $\boldsymbol{R}_{J}$ matrix solution, 


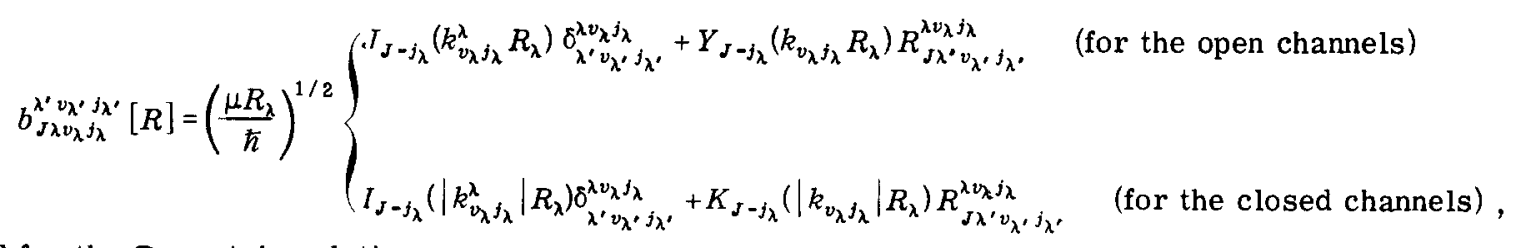

and for the $\mathbf{S}_{\boldsymbol{j}}$ matrix solution

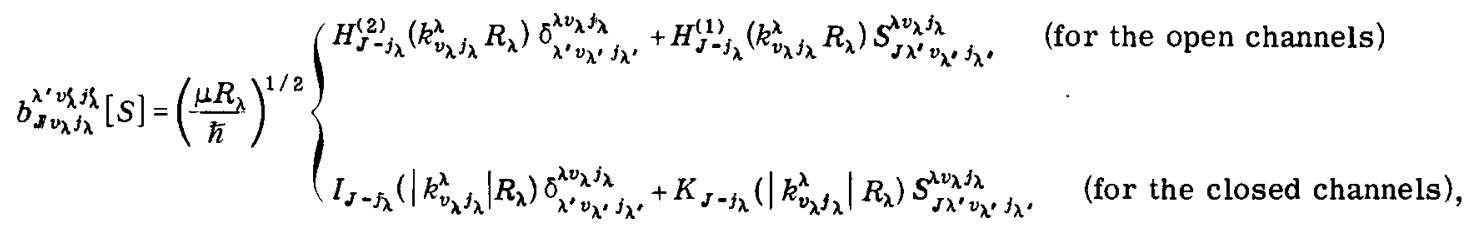

and the Hankel functions $H_{l}$ are related to the Bessel functions $J_{l}, Y_{l}$ by

$$
H_{l}^{(1,2)}=J_{1} \pm i Y_{1}
$$

The asymptotic sign in Eq. (5.4) signifies that for each $\lambda$ in the summation the corresponding $R_{\lambda}$ goes to infinity. The $\boldsymbol{R}_{J}$ and $\boldsymbol{S}_{J}$ matrices defined in Eqs. (5.5) and (5.6) have dimension $3 N \times 3 N$ and include both open and closed channel initial and final states. Only the open $\rightarrow$ open transitions have direct physical significance, and we will label the submatrices formed by the corresponding matrix elements by the symbols $\mathbf{R}_{J}^{0}$ and $\mathbf{S}_{J}^{0}$. It is these open channel matrices $\mathbf{R}_{J}^{0}$ and $\mathbf{S}_{J}^{0}$ which obey the usual properties that $\mathbf{R}_{J}^{0}$ is real and symmetric and $\mathbf{S}_{J}^{0}$ is unitary and symmetric, ${ }^{24}$ as a result of the time reversal invariance and conservation of flux properties of the Schrödinger equation. Actually, Eqs. (5.4)-(5.7) are expressed in terms of the mass-scaled variables $r_{\lambda}, R_{\lambda}$ of Eqs. (2.2a) and (2. $2 \mathrm{~b})$, whereas the reactive and scattering matrices we are interested in are defined in terms of equivalent expressions involving the unscaled $\overline{\mathbf{r}}_{\lambda}, \overline{\mathbf{R}}_{\lambda}$. However, Eq. (5.4) and its unscaled variable counterpart are proportional to one another, and as a result $\mathbf{R}_{\boldsymbol{J}}$ and $\mathbf{S}_{\boldsymbol{J}}$ are invariant under the $\mathbf{r}_{\lambda}, \mathbf{R}_{\lambda}-\overline{\mathbf{r}}_{\lambda}, \overline{\mathbf{R}}_{\lambda}$ transformation; this permits us to use directly the unbarred variable results to calculate these matrices. From Eqs. (5.7), Eqs. (5.5) and (5.6), we can obtain the following relation between the open channel subblocks of the reactance and scattering matrices, analogous to the one valid for three dimensions ${ }^{38,39}$ :

$$
\mathbf{S}_{J}^{0}=\left(\mathbf{I}-i \mathbf{R}_{J}^{0}\right)\left(\mathbf{I}+i \mathbf{R}_{J}^{0}\right)^{-1},
$$

where $I$ is the identity matrix. We will discuss the physical significance of the scattering matrix at the end of this section.

Let us indicate how $\mathbf{R}_{\boldsymbol{J}}$ can be obtained from the numerically determined solutions $\Psi_{J}^{(i) v j}$. We rewrite Eqs. (5.3) and (5.5) in $3 N \times 3 N$ matrix form as

$$
\mathrm{e}_{J}=g_{J}^{+} \mathrm{C}_{J}^{+}+\mathrm{g}_{J}^{-} \mathrm{C}_{J}^{-}
$$

and

$$
\mathbf{b}_{J}=\mathbf{V}^{-1 / 2}\left(\mathbf{J}_{J}+\mathbf{Y}_{J} \tilde{\mathbf{R}}_{J}\right),
$$

where

$$
\left(\mathbf{g}_{J}^{ \pm}\right)_{\lambda v_{\lambda} j_{\lambda}}^{\lambda^{\prime} v_{\lambda^{\prime}} j_{\lambda^{\prime}}}=\delta_{\lambda \lambda^{\prime}} g_{J v_{\lambda^{j}} \lambda_{\lambda}}^{\lambda^{\prime} v_{\lambda^{\prime} j_{\lambda^{\prime}}}}
$$

$$
\begin{aligned}
& \left(\mathbf{V}^{-1 / 2}\right)_{\lambda v_{\lambda} j_{\lambda}}^{\lambda v_{\lambda^{\prime}} j_{\lambda^{\prime}}}=\delta_{\lambda \lambda^{\circ}} \delta_{v_{\lambda^{\prime}} j_{\lambda}}^{v_{\lambda^{\prime} j^{\prime}}}\left(V_{v_{\lambda} j_{\lambda}}^{\lambda}\right)^{-1 / 2}, \\
& V_{v_{\lambda} j_{\lambda}}^{\lambda}=\hbar k_{v_{\lambda} j_{\lambda}}^{\lambda} / \mu \\
& \left(J_{J}\right)_{\lambda v_{\lambda} j_{\lambda}}^{\lambda^{\prime} v_{\lambda^{\prime} j_{\lambda^{\prime}}}}=\delta_{\lambda \lambda^{\prime}} \delta_{\substack{v_{\lambda} j_{\lambda} \\
v_{\lambda} j_{\lambda^{\prime}}}}\left(\left|k_{v_{\lambda} j_{\lambda}}\right| R_{\lambda}\right)^{1 / 2} \\
& \times \begin{cases}J_{J-j_{\lambda}}\left(k_{v_{\lambda} j_{\lambda}}^{\lambda} R_{\lambda}\right) & \text { (open channels) } \\
I_{J-j_{\lambda}}\left(\left|k_{v_{\lambda} j_{\lambda}}^{\lambda}\right| R_{\lambda}\right) & \text { (closed channels), }\end{cases} \\
& \left(\mathbf{Y}_{J}\right)_{\lambda v_{\lambda} j_{\lambda}}^{\lambda^{\prime} v_{\lambda^{\prime}} j_{\lambda^{\prime}}}=\delta_{\lambda \lambda^{\prime}} \delta_{v_{\lambda} j_{\lambda}}^{v_{\lambda} j_{\lambda^{\prime}}}\left(\left|k_{v_{\lambda} j_{\lambda}}^{\lambda}\right| R_{\lambda}\right)^{1 / 2} \\
& \times \begin{cases}Y_{J-j_{\lambda}}\left(k_{v_{\lambda} j_{\lambda}}^{\lambda} R_{\lambda}\right) & \text { (open channels) } \\
K_{J-j_{\lambda}}\left(\left|k_{v_{\lambda} j_{\lambda}}\right| R_{\lambda}\right) & \text { (closed channels) }\end{cases}
\end{aligned}
$$

and $\tilde{\mathbf{R}}_{J}$ is the transpose of $\mathbf{R}_{J}$. The elements of the $e_{J}$ and $\mathbf{b}_{J}$ matrices are the $e_{J \lambda v_{\lambda} j_{\lambda}}^{(i) v j}$ and $b_{J \lambda v_{\lambda} j_{\lambda}}^{(i) j_{j}}[R]$, respectively. In all these matrix elements, the subscripts other than $J$ denote the row and the superscripts the column to which they belong. The reactance matrix

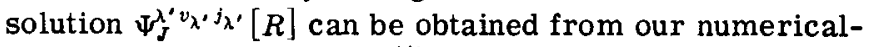
ly determined solutions $\Psi_{J}^{(i) v j}$ by taking linear combinations of the latter:

$$
\Psi_{J}^{\lambda^{\prime} v_{\lambda^{\prime}} j \lambda^{\prime}}[R]=\sum_{i=1}^{3} \sum_{v f} \Psi_{J}^{(i) v j} Q_{(i) v j^{j}}^{J \lambda^{\prime} v \lambda^{\prime}} .
$$

As was mentioned at the end of Sec. IV, the matching equation [Eq. (4.44)] determines $\mathbf{C}_{J}^{*}\left(\mathbf{C}_{J}^{-}\right)^{-1}$ but not $\mathbf{C}_{J}^{+}$ or $C_{j}^{-}$individually. To complete their evaluation we choose $Q_{(i) v j}^{J \lambda^{\prime} v \lambda^{j} \lambda_{\lambda^{\prime}}}=\delta_{(i) v j}^{\lambda^{\lambda} v v_{\lambda^{\prime}} j_{\lambda^{\prime}}}$ in Eq. (5.15), which is equivalent to requiring that the $C_{J}^{ \pm}$produce solutions which are not only smoothly matched, but satisfy the reactance asymptotic conditions as well. (It will soon become apparent that there are enough degrees of freedom left over to permit this condition to be imposed.) We then substitute Eqs. (5.2) and (5.4) into Eq. (5.15) and equate coefficients of terms having the same arrangement channel $\lambda$ and vibration-rotation basis functions $r_{\lambda}^{-1 / 2} \varphi_{j_{\lambda}}\left(\gamma_{\lambda}\right) \phi_{v_{\lambda} j_{\lambda}}^{\lambda}\left(r_{\lambda}\right)$. Expressing the resulting equations in matrix form and using Eqs. (5.9) and (5.10), we obtain

$$
\left(\mathbf{g}_{J}^{+} \mathbf{C}_{J}^{*}+\boldsymbol{g}^{-} \mathbf{C}_{J}^{J}\right)=\mathbf{V}_{-1 / 2}\left(J_{J}+\mathbf{Y}_{J} \tilde{\mathbf{R}}_{J}\right)
$$

The analogous equation for the derivative $\left(R_{\lambda}\right)^{-1 / 2}(\theta /$ $\left.\theta R_{\lambda}\right)\left[\left(R_{\lambda}\right)^{1 / 2} \Psi_{J}[R]\right]$ is

$$
\left(\mathbf{g}_{J}^{+} \mathbf{C}_{J}^{+}+\mathbf{g}_{J}^{\prime} \mathbf{C}_{J}^{-}\right)=\mathbf{V}^{-1 / 2}\left(\mathbf{J}_{J}^{\prime}+\mathbf{Y}_{J}^{\prime} \tilde{\mathbf{R}}_{J}\right),
$$

where prime denotes differentiation with respect to $R_{\lambda}$, with $\lambda=\alpha, \beta, \gamma$ as appropriate. These last two equa- 
tions and Eq. (4.44) can then be simultaneously solved to yield the following expression for $\overline{\mathbf{R}}_{J}$ :

$$
\begin{aligned}
\overline{\mathbf{R}}_{J}= & -\mathbf{V}^{1 / 2} \mathbf{W}_{J}^{-1}\left[\left(\mathbf{J}_{J}^{\prime} \mathbf{g}_{J}^{+}\right)\left(\mathbf{N}_{J}^{+}\right)^{-1} \mathbf{N}_{J}^{-}-\left(J_{J}^{\prime} \mathbf{g}_{J}^{-}-\mathbf{J}_{J} \mathbf{g}_{J}^{-\prime}\right)\right] \\
& \times\left[\left(\mathbf{Y}_{J}^{\prime} \mathbf{g}_{J}^{+}-\mathbf{Y}_{J} \mathbf{g}_{J}^{+}\right)\left(\mathbf{N}_{J}^{+}\right)^{-1} \mathbf{N}_{J}^{-}-\left(\mathbf{Y}_{J}^{\prime} \mathbf{g}_{J}^{-}-\mathbf{Y}_{J} \mathbf{g}_{J}^{-\prime}\right]^{-1} \mathbf{W}_{j} \mathbf{V}^{-1 / 2}\right.
\end{aligned}
$$

where

$$
\mathbf{W}_{J}=\mathbf{Y}_{J}^{\prime} \mathbf{J}_{J}-\mathbf{J}_{J}^{\prime} \mathbf{Y}_{J}
$$

is the diagonal matrix whose diagonal elements are the Wronskians of the regular and irregular ordinary or modified Bessel functions. ${ }^{33}$ None of the matrices whose inverses appear in Eq. (5.18) are in general singular. Transposition of Eq. (5.18) gives $\mathbf{R}_{J}$, and Eqs. (5.18) and (4.44) when substituted into Eqs. (5.16) or (5.17) permit an explicit and unique determination of $\mathbf{C}_{J}^{+}$and $\mathbf{C}_{J}^{-}$.

The procedure just described furnished the full $\mathbf{R}_{J}$ matrix. Its closed channel parts may now be discarded and the open channel part of the scattering matrix $\mathbf{S}_{J}^{0}$ may then be used to calculate $P_{J \lambda v_{\lambda} j_{\lambda}}^{\lambda^{\prime} v_{\lambda^{*}} j_{\lambda^{*}}}$, the probability of transition from a given initial arrangement channel $\lambda^{\prime}$ and internal state $v_{\lambda^{\prime}} j_{\lambda^{\prime}}$ (for a given total angular momentum quantum number $J$ ) through the relation

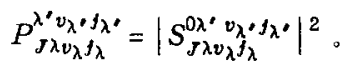

Note that this is a distinguishable-atom transition probability。 Effects of indistinguishability of particles will be considered in Sec. VI. B. As mentioned in Sec. II. C, the total angular momentum is simply equal to the algebraic sum of the rotational and orbital angular momenta, $i_{\circ} e_{\rho}, J=j_{\lambda}+l_{\lambda}$, which allows us to re-express $P_{\delta \lambda^{\prime} v_{\lambda^{\prime}} J_{\lambda}}^{\lambda^{\prime}}$ in terms of initial and final orbital angular momenta $l_{\lambda}$ and $l_{\lambda^{\prime}}$. This has a useful semiclassical interpretation since the initial orbital angular momentum quantum number $l_{\lambda}$ is related to the classical impact parameter $b_{\lambda}$ through the relation

$$
l_{\lambda}=\bar{k}_{v_{\lambda} j_{\lambda}}^{\lambda} b_{\lambda} \text {, }
$$

where $\bar{k}_{v_{\lambda} j_{\lambda}}^{\lambda}$ is the wave number in the $\bar{R}_{\lambda}, \bar{r}_{\lambda}$ coordinate system (defined in Sec。 II $\mathrm{S}_{\mathrm{B}}$ )

$$
\begin{aligned}
\bar{k}_{v_{\lambda} j_{\lambda}}^{\lambda} & =\left(\frac{2 \mu_{\lambda, v_{k}}}{\hbar^{2}}\left(E-\epsilon_{v_{\lambda \lambda \lambda}}^{\lambda}\right)^{1 / 2}\right. \\
& =a_{\lambda} k_{v_{\lambda} j_{\lambda}}^{\lambda}
\end{aligned}
$$

\section{B. Distinguishable-atom scattering amplitudes and cross sections}

In this section we define a coplanar dimensionless distinguishable-atom scattering amplitude and give its relation to the coplanar scattering matrix and differential and integral cross sections.

Let $\Psi^{\lambda v}{ }^{j} \lambda[P]$ be a "physical" solution to the Schrödinger equation ( 2.4 ), involving the mass-scaled coordinates $\mathrm{r}_{\lambda}, R_{\lambda}$, which behaves asymptotically at large $R_{\lambda}, R_{\nu}$, and $R_{\kappa}$ as

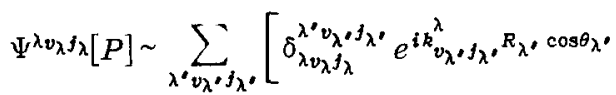

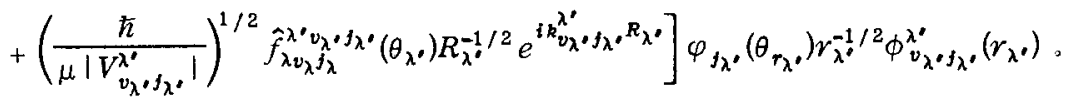

This solution describes a collision between atom $A_{\lambda}$ and molecule $A_{\nu} A_{K}$ in internal vibrational-rotational state $v_{\lambda} j_{\lambda}$, with the relative initial velocity of colliding partners, in scaled coordinates, being $V_{v_{\lambda} j_{\lambda}}^{\lambda}$. The direction of relative approach of the colliding partners is the $x$ axis of Fig. 1. The scaled coordinate relative motion wave number $k_{v_{\lambda} \rho_{\lambda^{\prime}}}^{\lambda^{\prime}}$ is real positive for open channels and imaginary positive for closed ones. The first and second $R_{\lambda^{\prime}}$-dependent terms in $\mathrm{Eq}$. $(5,23)$ represent, respectively, the incident relative motion line wave and the scattered circular wave, which can be nonreactive $\left(\lambda^{\prime}=\lambda\right)$ or reactive $\left(\lambda^{\prime} \neq \lambda\right)$. These are the $2 \mathrm{D}$ analogues of the 3D incident plane wave and scattered spherical waves. The factor outside the square brackets is the planar-motion vibrational-rotational wavefunction of the isolated $\mathrm{A}_{\nu^{\prime}} A_{K^{\prime}}$ molecule in state $v_{\lambda^{\prime}}, j_{\lambda^{\prime}}$, in scaled coordinates.

Equation (5.23) defines a set of dimensionless distinguishable atom scattering amplitudes $\hat{f}\left(\theta_{\lambda^{\prime}}\right)$. The differential cross section for the $\lambda v_{\lambda} j_{\lambda}-\lambda^{\prime} v_{\lambda^{\prime}} j_{\lambda^{\prime}}$ process (assuming that both these states are open at the total energy $E$ being considered) is related to the corresponding $\hat{f}$ by

$$
{ }_{\lambda v_{\lambda} \lambda_{\lambda}}^{\lambda^{\prime} v_{\lambda^{\prime}} j_{\lambda^{\prime}}}\left(\theta_{\lambda^{\prime}}\right)=\left(1 / \bar{k}_{v_{\lambda^{j}} j_{\lambda}}^{\lambda}\right)\left|\hat{f}_{\lambda v_{\lambda} J_{\lambda}}^{\lambda^{\prime} v_{\lambda^{\prime}} f_{\lambda^{\prime}}}\right|^{2},
$$

where $\bar{k}_{v_{\lambda, \lambda}}^{\lambda}$ is the unscaled initial relative motion wave number of the colliding particles. For either reactive or nonreactive collisions, $\theta_{\lambda}$, is the angle between the initial and final velocity vectors of the atom with respect to the diatom in the center of mass system Oxy of Fig. 1. The integral cross section for the same process is

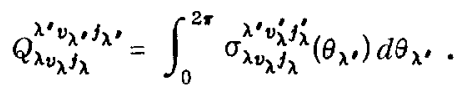

$\sigma$ and $Q$ have dimensions of a length per unit angle and of a length, respectively.

We now relate the scattering amplitudes to the scattering matrix. To do this, we expand the physical solution Eq. $(5,23)$ in terms of the scattering matrix solution [Eqs. $(5,4)$ and $(5,6)]$ using an equation analogous to $(2,12)$ :

$$
\Psi^{\lambda v_{\lambda} J_{\lambda}}[P]=\sum_{J=-\infty}^{\infty} C_{J}^{\lambda v_{\lambda} j j_{\lambda}} \Psi_{J}^{\lambda v \lambda j \lambda}[S] .
$$

To find expressions for the coefficients $C_{J}^{\lambda_{\lambda} j_{\lambda}}$ and the scattering amplitudes $\hat{f}_{\lambda_{\eta} \lambda^{\prime} f_{\lambda}}^{\lambda^{\prime} \lambda^{\prime}}$, we first expand the $\theta_{\lambda^{\prime}}$ dependent portions of $\mathrm{Eq}$. $(5.23)$ in terms of the eigenfunctions $\varphi_{J}\left(\theta_{\lambda^{\prime}}\right)$, then express the $R_{\lambda^{\prime}}$-dependent portions of Eqs. $(5,23)$ and $(5,6)$ in their asymptotic forms 
[Eq. (3.27)] involving exponentials 。 We finally equate the coefficients of the corresponding $R_{\lambda}$, exponentials, $\varphi_{J}\left(\theta_{\lambda^{\prime}}\right)$ and rotation-vibration basis functions in both sides of $\mathrm{Eq} .(5,26)$ and solve the resulting equations for $C_{J}^{\lambda_{\lambda} J_{\lambda}}$ and for the coefficients of the expansion of $\hat{f}_{\lambda v_{\lambda} j_{\lambda} \lambda^{\prime} \nu^{\prime} j_{\lambda^{\prime}}}\left(\theta_{\lambda^{\prime}}\right)$ in the $\varphi_{J}\left(\theta_{\lambda^{\prime}}\right)$ 。

The expansion for the line wave is ${ }^{40}$

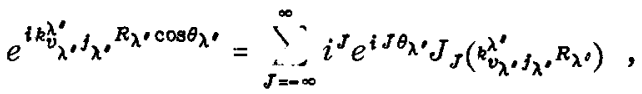

$$
\begin{aligned}
& \underset{R_{\lambda^{\prime}-\infty}}{\sim} \frac{1}{k_{v \lambda^{\prime} j_{\lambda^{\prime}}}^{\lambda^{\prime}} R_{\lambda^{\prime}}} \varlimsup_{j=-\infty}^{\infty}\left[e^{i k_{\lambda^{\prime}}^{\lambda^{\prime}} j_{\lambda^{\prime}} R_{\lambda^{\prime}}} e^{-i(x / 4)}\right. \\
& \left.+(-1)^{J} e^{-i k_{\nu_{\lambda^{\prime}}^{\lambda^{\prime}} j_{\lambda^{\prime}} R_{\lambda^{\prime}}}} e^{i(\pi / 4)}\right] \varphi_{J}\left(\theta_{\lambda^{\prime}}\right)
\end{aligned}
$$

As a result of the relation between $\gamma_{\lambda}, \theta_{\lambda}$, and $\theta_{r_{\lambda}}$ given after $\mathrm{Eq} .(2,1)$, and of $\mathrm{Eq} .(2.9)$, we have

$$
\varphi_{j_{\lambda^{\circ}}}\left(\theta_{\gamma_{\lambda}}\right)=(2 \pi)^{1 / 2} \varphi_{j \lambda^{\circ}}\left(\theta_{\lambda^{\prime}}\right) \varphi_{J_{\lambda^{\prime}}}\left(\gamma_{\lambda^{\prime}}\right) \text { 。 }
$$

Using Eqs。(5.26)-(5.28) and following the procedure outlined above, we get

$$
C_{J}^{\lambda v_{\lambda} j_{\lambda}}=\left(\frac{\hbar \pi}{2 \mu}\right)^{1 / 2} \exp \left[i\left(J-j_{\lambda}\right) \frac{1}{2} \pi\right]
$$

and, for the dimensionless scattering amplitudes to open channels,

$$
\begin{aligned}
& \hat{f}_{\lambda v_{\lambda} j_{\lambda}^{\prime}}^{\lambda^{\prime} v^{\prime} j_{\lambda^{\prime}}}\left(\theta_{\lambda^{\prime}}\right)=\exp \left(-i j_{\lambda^{\prime}} \theta_{\lambda^{\prime}}\right) \exp \left[i\left(j_{\lambda^{\prime}}-j_{\lambda}-\frac{1}{2}\right) \frac{1}{2} \pi\right] \\
& \times \sum_{J=-\infty}^{\infty}\left(S_{J \lambda v \lambda_{\lambda} J_{\lambda}}^{0 \lambda^{\prime} v_{\lambda^{\prime}} J_{\lambda^{\prime}}}-\delta_{\lambda v \lambda_{\lambda} J_{\lambda}}^{\lambda^{\prime} v^{\prime} f_{\lambda^{\prime}}}\right) \varphi_{J}\left(\theta_{\lambda^{\prime}}\right)
\end{aligned}
$$

Equation $(5,30)$ differs from that obtained by Walker and Wyatt ${ }^{41}$ only by the phase factor $\exp \left(-i j_{\lambda^{\prime}}, \theta_{\lambda^{\prime}}\right)$. Substitution of $\mathrm{Eq} .(5.30)$ into (5.24) and of the result into Eq. $(5,25)$ leads to the following rather simple expression for the integral cross section:

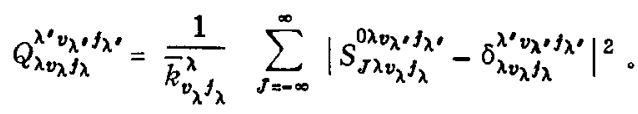

Equations $(5.35)$ and $(5.36)$ may be written in terms of a sum over $J$ from 0 to $\infty$ by using the relation

$$
S_{J \lambda v_{\lambda} f_{\lambda}}^{0 \lambda^{\prime} v_{\lambda^{\prime}} J_{\lambda^{\prime}}}=S_{-J \lambda v_{\lambda}-j_{\lambda}}^{0 \lambda^{\prime} v_{\lambda^{\prime}}-f_{\lambda^{\prime}}} .
$$

This expression is a consequence of the symmetry of the Hamiltonian with respect to reflection through the plane of motion. Additional symmetry relations which follow from $\mathrm{Eq} .(5.32)$ are

$$
\hat{f}_{\lambda v_{\lambda}-j_{\lambda}}^{\lambda v^{\prime} v_{\lambda}-f_{\lambda^{\prime}}}\left(2 \pi-\theta_{\lambda^{\prime}}\right)=\hat{f}_{\lambda v_{\lambda} j_{\lambda}^{\prime}}^{\lambda^{\prime} v_{\lambda^{\prime}} j_{\lambda^{\prime}}}\left(\theta_{\lambda^{\prime}}\right)
$$

and

$$
Q_{\lambda v_{\lambda}-j_{\lambda}}^{\lambda^{\prime} v_{\lambda^{\prime}-f_{\lambda^{\prime}}}}=Q_{\lambda v_{\lambda} j_{\lambda}}^{\lambda^{\prime} v \nu^{\prime} f_{\lambda^{\prime}}}
$$

Equations $(5,32)$, through $(5,34)$ are valid for any planar atom plus diatomic molecule collision process. For reactions of higher symmetry such as $\mathrm{H}+\mathrm{H}_{2}$, there exist additional relationships, some of which will be discussed in the next section.

\section{APPLICATION TO PLANAR $\mathrm{H}+\mathrm{H}_{2}$}

A. The integration, matching, and distinguishable-atom asymptotic analysis

In the application of the methods described in Sec. III and IV to the $\mathrm{H}+\mathrm{H}_{2}$ exchange reaction, a considerable reduction in computation time can be realized by utilizing two important symmetry properties of this collision system. The first is the invariance of the collision system and associated coordinate systems with respect to a cyclic permutation of the three atoms. Mathematically this means that all equations derived in Secs. III-V are invariant to a cyclic permutation of the indices $\lambda \nu \kappa$, which implies (1) that we need to integrate the Schrödinger equation in only one of the three arrangement channel regions depicted in Fig。2, (2) that we need only calculate the projection coefficients $\bar{h}, \bar{h}^{\prime}, \bar{f}$, and $\bar{f}^{\prime}$ defined in Eqs. $(4.11),(4.12)$, $(4.22)$, and $(4.23)$ on one of the three matching surfaces (such as $\pi_{\nu \lambda}$ ), and (3) that the $\lambda \rightarrow \nu, \nu \rightarrow \kappa$, and $\kappa-\lambda$ distinguishable-atom scattering amplitudes are all identical, as are $\nu \rightarrow \lambda, \lambda \rightarrow \kappa, \kappa \rightarrow \nu$, and $\lambda \rightarrow \lambda, \nu$ $\rightarrow \nu, \kappa-\kappa$, so we may restrict ourselves to a calculation of the $\lambda-\lambda, \lambda-\nu$, and $\lambda \rightarrow \kappa$ scattering amplitudes only. The second symmetry property is related to the invariance of the collision system (but not the associated coordinate systems) with respect to an interchange of any two of the three atoms. This results in a potential function $V^{\lambda}\left(r_{\lambda}, R_{\lambda}, \gamma_{\lambda}\right)$ which is symmetric about $\gamma_{\lambda}=\pi /$ 2 and $3 \pi / 2$ :

$V^{\lambda}\left(r_{\lambda}, R_{\lambda}, \pi-\gamma_{\lambda}\right)=V^{\lambda}\left(r_{\lambda}, R_{\lambda}, \gamma_{\lambda}\right) \quad$ for $0 \leqslant \gamma_{\lambda} \leqslant \pi$, $V^{\lambda}\left(r_{\lambda}, R_{\lambda}, 3 \pi-\gamma_{\lambda}\right)=V^{\lambda}\left(r_{\lambda}, R_{\lambda}, \gamma_{\lambda}\right) \quad$ for $\pi<\gamma_{\lambda}<2 \pi$ 。

To a large extent, the consequences of this property depend on the coordinate system being used, for while $E q_{0}(6,1)$ is valid in all regions of configuration space, the $\lambda$ arrangement channel coordinates $r_{\lambda}, R_{\lambda}, \gamma_{\lambda}$ are not the most convenient coordinates to use in all three arrangement channel regions. In arrangement channel region $\lambda, \mathrm{Eq}_{0}(6.1)$ has the immediate effect of decoupling rotational states of even and odd quantum numbers $j_{\lambda}$. This means that the integration in arrangement channel region $\lambda$ can be done in two separate steps, one for even $j_{\lambda}$ and one for odd $j_{\lambda}$. In each of these steps the total number of basis functions required is only about half of that needed in the absence of this decoupling. Since the computation time varies as $N^{a}$, where $N$ is the number of states being integrated and $a$ is 2 for $N<10$ and 3 for $N>20,{ }^{20}$ we see that a saving of factors of 2 to 4 in computation time may be realized by this decoupling. In a similar manner, the calculation of the matching surface coefficients of Eqs。(4.11), (4.12), (4.22), and $(4.23)$ may be done in two separate steps, one for even $\mathbf{j}_{\lambda}$ and one for odd $\mathbf{j}_{\lambda}$, and the coefficient matrices $f_{J}^{\nu_{t}}$ and $f^{\nu_{ \pm}}$may be obtained from $h_{J}^{\lambda_{t}}$ and $h_{J}^{\prime \lambda_{t}}$ by using the simple relations

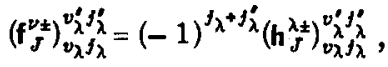

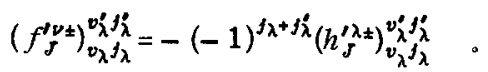

We must note, however, that the number of matching surface functions $B_{v_{\lambda} j_{\lambda}}^{\nu \lambda}$ is still $N / 2$, where $N$ is the total 
number of even plus odd $j_{\lambda}$ states. The matching procedure ultimately couples the even and odd rotational states $j_{\lambda}$ [through Eq. (4.44)] so that decoupling beyond that point is lost. However, symmetry of the system about $\gamma_{\lambda}=\pi / 2$ and $3 \pi / 2\left[E_{0},(6,1)\right]$ may be used to relate the $\lambda \rightarrow \nu$ and $\lambda \rightarrow \kappa$ scattering amplitudes according to

$$
\hat{f}_{\lambda \nu j}^{\nu v^{\prime} f^{\prime}}=(-1)^{f+j^{\prime}} \hat{f}_{\lambda v j}^{v^{\prime} f^{\prime \prime}} \text {, }
$$

as is shown in Appendix C. Equation $(6,3)$ may be used to reduce the work involved in the asymptotic analysis to the calculation of only the $\lambda \rightarrow \lambda$ and $\lambda \rightarrow \nu$ scattering amplitudes. An additional consequence of $\mathrm{Eq}_{0}(6,1)$ valid only for the $\lambda-\lambda$ scattering amplitudes ( $i, e_{o}$, the nonreactive transitions) is the familiar relation (also derived in Appendix C)

$$
\hat{f}_{\lambda \nu j}^{\lambda \nu^{\prime} j^{\prime}}=0 \text { for } j-j^{\prime}=\text { odd , }
$$

and the incorporation of this relation into the asymptotic analysis can also result in a reduction of computational effort. We should note, however, that the two symmetries given by Eqs。 $(6,3)$ and $(6,4)$ depend on our use of a complete basis set $B_{v_{\lambda} j_{\lambda}}^{v \lambda}$ for expanding the wavefunction on the matching surface and therefore may be used as a test of the convergence of the method provided these symmetries are not built into the calculations.

\section{B. Postantisymmetrization}

Up to this point, we have considered the three atoms to be distinguishable. However, to calculate physically measurable quantities such as cross sections for reactions like $\mathrm{H}+\mathrm{H}_{2}$, we must include for effects due to indistinguishability of the three atoms and the Pauli principle. This means that the physically meaningful solutions to the Schrödinger equation must be antisymmetric with respect to interchange of hydrogen nuclei (which have spin $\frac{1}{2}$ )。 Some conceptual difficulties are occasionally encountered when using time-independent solutions, since antisymmetrization seems to imply incoming waves in all three arrangement channels asymptotically. A consideration of the time-dependent wavepacket generated from the time-independent solutions resolves this, however. As Taylor has shown, ${ }^{42}$ the asymptotic indistinguishable-atom wave packet is identical to its distinguishable-atom counterpart, so that at any given time before the collision, the incoming wave is localized in a single distinguishable-atom arrangement channel. Once the collision begins, such localization is lost and the concept of distinguishableatom arrangement channels becomes meaningless.

There is actually a number of ways by which indistinguishable-atom scattering amplitudes may be obtained from time-independent wavefunctions:

1. We can preantisymmetrize the wavefunction, and solve the coupled integrodifferential equations generated when this wavefunction is substituted into the Schrödinger equation. Such a procedure was used by Wolken and Karplus ${ }^{16}$ and the resulting solutions yield directly the indistinguishable-atom scattering amplitudes.

2. We can solve the Schrödinger equation using the method outlined in this paper for distinguishable particles, antisymmetrize the resultant primitive wavefunctions, and use the asymptotic form of these antisymmetrized wavefunctions to obtain the indistinguishable-atom scattering amplitudes. A related procedure was considered by Truhlar and Abdallah ${ }^{43}$ in their method for studying rearrangement collisions.

3. We may use the distinguishable-atom primitive wavefunctions generated in (2) to determine the distinguishable-atom scattering amplitudes. These amplitudes may then be linearly combined to yield the corresponding indistinguishable-atom amplitudes. This is the usual procedure of postantisymmetrization ${ }^{23}$ which we have used and which is described for the case of hydrogen atom exchange in Appendix D.

If exact solutions to the Schrödinger equation are used, then all three methods should give the same results, but the use of approximate solutions can lead to different results even when comparing methods (2) and (3) where the same distinguishable-atom primitive wavefunctions are used An example of this arises when distinguishable-atom solutions that do not obey microscopic reversibility are considered. In this case the process of antisymmetrization of the wavefunction is not interchangeable with that of extracting the asymptotic form of the wavefunction, thus leading to different results when methods (2) and (3) are applied. Since the numerical method described in this paper is designed to provide accurate solutions to the Schrödinger equation for distinguishable atoms, the differences between methods (2) and (3) are of secondary importance and we shall restrict ourselves to considering method (3) for the remainder of this paper. The resulting expressions for the indistinguishableparticle transition amplitudes in terms of their distinguishable-atom counterparts, as described in Appendix D and also by Doll, George, and Miller, ${ }^{44}$ are summarized below (where the direct and exchange amplitudes $\hat{f}_{1}^{1}$ and $\hat{f}_{2}^{2}$ of that Appendix are relabeled as $\hat{f}_{\lambda}^{\lambda}$ and $\hat{f}_{\lambda}^{v}$, respectively):

(a) para $\rightarrow$ para:

$$
\sigma_{p v j}^{p v^{\prime} j^{\prime}}(\theta)=\frac{1}{\bar{k}_{v j}^{\lambda}}\left|\hat{f}_{\lambda v j}^{\lambda v^{\prime} f^{\prime}}(\theta)-\hat{f}_{\lambda v f}^{\nu v^{\prime} j^{\prime}}(\theta)\right|^{2},
$$

(b) para - ortho:

$$
\sigma_{p v j}^{o v^{\prime} j^{\prime}}(\theta)=\frac{3}{\bar{k}_{v j}^{\lambda}}\left|\hat{f}_{\lambda v j}^{v v^{\prime} j^{\prime}}(\theta)\right|^{2}
$$

(c) ortho-para:

$$
\sigma_{o v j^{p}}^{p^{\prime} j^{\prime}}(\theta)=\frac{1}{\bar{k}_{v f}^{\lambda}}\left|\hat{f}_{\lambda y j}^{\nu v^{\prime} j^{\prime}}(\theta)\right|^{2},
$$

(d) ortho - ortho:

$$
\begin{aligned}
\sigma_{v v f}^{o v^{\prime} j^{\prime}}(\theta)=\frac{1}{\bar{k}_{v f}^{\lambda}} & {\left[\left|\hat{f}_{\lambda v f^{\prime}}^{\lambda v^{\prime \prime}}(\theta)+\hat{f}_{\lambda v f^{\prime}}^{\nu v^{\prime} f^{\prime}}(\theta)\right|^{2}\right.} \\
& \left.+2\left|\hat{f}_{\lambda v f}^{\nu v^{\prime \prime} f^{\prime \prime}}(\theta)\right|^{2}\right] .
\end{aligned}
$$

Note that there is no reference to arrangement channel on the left sides of Eqs. (6,5) since this distinction has no meaning after the effect of the Pauli principle is 
included. The corresponding integral cross sections are

(a) para $\rightarrow$ para:

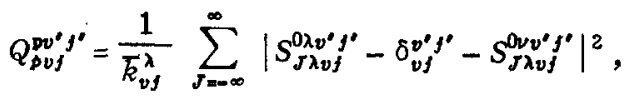

(b) para $\rightarrow$ ortho:

$Q_{p v f^{\prime}}^{o v^{\prime} j^{\prime}}=\frac{3}{\bar{k}_{v f}^{\lambda}} \sum_{J=-\infty}^{\infty}\left|S_{J \lambda v f^{0 \lambda v^{\prime}}}^{0,}\right|^{2}$,

(c) ortho-para:

$Q_{o v j^{\prime}}^{p v^{\prime} j^{\prime}}=\frac{1}{\bar{k}_{v j}^{\lambda}} \sum_{J=-\infty}^{\infty}\left|S_{J \lambda v j^{0}}^{0 v v^{\prime} j^{\prime}}\right|^{2}$,

(d) ortho $\rightarrow$ ortho:

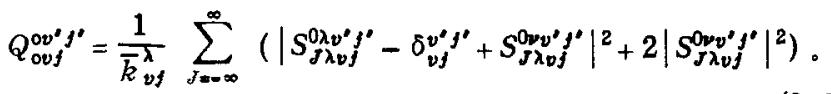

As expected, the para - ortho and ortho $\rightarrow$ para cross sections are simply proportional to the distinguishableatom reactive cross sections since only exchange scattering amplitudes contribute to them. These cross sections furnish direct information on the reactive process alone. The para - para and ortho - ortho cross sections will show effects due to the interference between the reactive and nonreactive $\left(i_{0} e_{0}\right.$, direct and exchange) scattering amplitudes. The interference effects should be most important when these two amplitudes have comparable magnitudes. We will discuss this interference phenomenon in more detail when presenting our results for planar $\mathrm{H}+\mathrm{H}_{2} \cdot{ }^{20}$ As pointed out in Appendix D, Eqs. (6.5) above are also valid for the three-dimensional $\mathrm{H}+\mathrm{H}_{2}$ reaction once the quantum numbers $m_{f}$ are added to the $v$ and $j$ ones and $\bar{k}_{v f}^{\lambda}$ is replaced by $\left(\bar{k}_{v j}^{\lambda}\right)^{2}$.

\section{ACKNOWLEDGMENTS}

One of us $(A, K$.$) wishes to thank Professor R. P$. Feynman for useful discussions on postantisymmetrization. He is also greatly indebted to Professor J.D. Roberts who, as Chairman of the Division of Chemistry and Chemical Engineering at Caltech during the crucial stages of this research, mustered the financial resources and offered the encouragement which were central to its successful completion. We also thank Professor Donald G. Truhlar for useful comments.

\section{APPENDIX A: THE $\lambda \rightarrow \nu$ TRANSFORMATION EQUATIONS AND RELATIONS ON THE MATCHING SURFACES}

In this Appendix we derive the important relations between the mass-scaled coordinates $\mathbf{R}_{\lambda}, \mathbf{r}_{\lambda}(\lambda=\alpha, \beta, \gamma)$ defined by Eqs. $(2,2)$. They permit us to change from coordinates appropriate for one arrangement channel to those appropriate for another one. We also examine the simplifications that occur when these relationships are evaluated on the matching surfaces defined by Eq. (3.2). This will allow us to prove that these surfaces are half-planes whose edge is the $O Y_{\lambda}$ axis in the $O X_{\lambda} Y_{\lambda} Z_{\lambda}$ space introduced in Sec. III. A. We will consider only the relationships between the arrangement channel coordinates $R_{\lambda}, r_{\lambda}$ and $R_{\nu}, r_{\nu}$ explicitly. The corresponding relationships between $R_{\nu}, r_{\nu}$ and $R_{k}, r_{k}$, and between $R_{k}, r_{k}$ and $R_{\lambda}, r_{\lambda}$ may be obtained by cyclic permutation of the indices $\lambda \nu \kappa$ 。

The following relations between the vectors $\overline{\mathbf{R}}_{\lambda}, \overline{\mathbf{r}}_{\lambda}$ and $\overline{\mathbf{R}}_{\nu}, \bar{r}_{\nu}$, valid for any configuration of the three atoms, follows from Fig. 1:

$$
\begin{aligned}
& \overline{\mathbf{R}}_{\nu}=-\overline{\mathbf{r}}_{\lambda}-\frac{m_{\lambda}}{m_{\lambda}+m_{\kappa}} \overline{\mathbf{r}}_{\nu} \\
& \overline{\mathbf{r}}_{\nu}=\overline{\mathbf{R}}_{\lambda}-\frac{m_{\nu}}{m_{\nu}+m_{k}} \bar{r}_{\lambda} .
\end{aligned}
$$

From these and Eqs. $(2.2)$ we get

$$
\left(\begin{array}{l}
R_{\nu} \\
r_{\nu}
\end{array}\right)=M\left(\begin{array}{l}
R_{\lambda} \\
r_{\lambda}
\end{array}\right),
$$

where $M$ is the $2 \times 2$ orthogonal matrix

$$
M=\left(\begin{array}{cc}
\cos \alpha_{\nu \lambda}-\sin \alpha_{\nu \lambda} \\
\sin \alpha_{\nu \lambda} & \cos \alpha_{\nu \lambda}
\end{array}\right),
$$

$\alpha_{\nu \lambda}$ being the angle between $\pi / 2$ and $\pi$ determined by

$$
\cos \alpha_{\nu \lambda}=-\left(\frac{m_{\lambda} m_{\nu}}{\left(m_{\lambda}+m_{\kappa}\right)\left(m_{\nu}+m_{\kappa}\right)}\right)^{1 / 2}
$$

and

$$
\sin \alpha_{\nu \lambda}=\left(\frac{m_{k} M}{\left(m_{\lambda}+m_{k}\right)\left(m_{k}+m_{\nu}\right)}\right)^{1 / 2}
$$

From these expressions we can get the equations for the $\theta_{\lambda}, R_{\lambda}, r_{\lambda}, \gamma_{\lambda}-\theta_{\nu}, R_{\nu}, r_{\nu}, \gamma_{\nu}$ transformation. Indeed, from Eqs. (A1) and (A2) and the definition of $\gamma_{\lambda}$ following Eq. $(2,1)$ we get

$$
\begin{aligned}
& R_{\nu}^{2}=\mathbf{R}_{\nu} \cdot \mathbf{R}_{\nu}=\cos ^{2} \alpha_{\nu \lambda} R_{\lambda}^{2}+\sin ^{2} \alpha_{\nu \lambda} r_{\lambda}^{2}-\sin 2 \alpha_{\nu \lambda} r_{\lambda} R_{\lambda} \cos \gamma_{\lambda}, \\
& r_{\nu}^{2}=r_{\nu} \cdot r_{\nu}=\sin ^{2} \alpha_{\nu \lambda} R_{\lambda}^{2}+\cos ^{2} \alpha_{\nu \lambda} r_{\lambda}^{2}+\sin 2 \alpha_{\nu \lambda} r_{\lambda} R_{\lambda} \cos \gamma_{\lambda},
\end{aligned}
$$

and therefore

$$
R_{\nu}^{2}+r_{\nu}^{2}=R_{\lambda}^{2}+r_{\lambda}^{2},
$$

which is a manifestation of the orthogonality of $\mathbf{M}_{0}$ In addition, it follows from Eqs. (A1) and (A2) that the cross products $\mathbf{R}_{\lambda} \times \mathbf{r}_{\lambda}$ and $\mathbf{R}_{\nu} \times \mathbf{r}_{\nu}$ are equal and therefore that

$$
R_{\nu} r_{\nu} \sin \gamma_{\nu}=R_{\lambda} r_{\lambda} \sin \gamma_{\lambda}
$$

Since $\gamma_{\nu}$ is in the range $0-2 \pi$, in order to have it completely specified we should obtain its cosine. Also from Eqs. (A4) and (A5) we get

$$
\begin{aligned}
\cos \gamma_{\nu} & =\frac{\mathbf{R}_{\nu} \cdot \mathbf{r}_{\nu}}{R_{\nu} r_{\nu}} \\
& =\frac{1}{R_{\nu} r_{\nu}}\left[\frac{1}{2} \sin 2 \alpha_{\nu \lambda}\left(R_{\lambda}^{2}-r_{\lambda}^{2}\right)+\cos 2 \alpha_{\nu \lambda} \cos \gamma_{\lambda} R_{\lambda} r_{\lambda}\right]
\end{aligned}
$$

Equations (A4), (A5), and (A7) completely describe the $R_{\lambda}, r_{\lambda}, \gamma_{\lambda}-R_{\nu}, r_{\nu}, \gamma_{\nu}$ transformation. To complete the $\lambda \rightarrow \nu$ transformation we define the angle $\Delta_{\nu \lambda}$,

$$
\Delta_{\nu \lambda}=\theta_{\nu}-\theta_{\lambda},
$$


and express it in terms of the $\lambda$ coordinates. We can write

$$
\begin{aligned}
& \mathbf{R}_{\lambda}=R_{\lambda}\left(\cos \theta_{\lambda} \hat{\mathbf{x}}+\sin \theta_{\lambda} \hat{\mathbf{y}}\right), \\
& \mathbf{r}_{\lambda}=r_{\lambda}\left[\cos \left(\theta_{\lambda}+\gamma_{\lambda}\right) \hat{\mathbf{x}}+\sin \left(\theta_{\lambda}+\gamma_{\lambda}\right) \hat{\mathbf{y}}\right],
\end{aligned}
$$

where $\hat{\mathbf{x}}$ and $\hat{\mathbf{y}}$ are the unit vectors along the laboratoryfixed axis depicted in Fig. 1. From these expressions, their $\nu$ counterpart and Eqs. (A1) and (A2) we can easily obtain the following expressions which determine $\Delta_{\nu \lambda}$ modulo $2 \pi$ :

$$
\begin{aligned}
& R_{\nu} \cos \Delta_{\nu \lambda}=R_{\lambda} \cos \alpha_{\nu \lambda}-r_{\lambda} \sin \alpha_{\nu \lambda} \cos \gamma_{\lambda}, \\
& R_{\nu} \sin \Delta_{\nu \lambda}=-r_{\lambda} \sin \alpha_{\nu \lambda} \sin \gamma_{\lambda} .
\end{aligned}
$$

We see that $\Delta_{\nu \lambda}$ is independent of $\theta_{\lambda}$ and a function of $R_{\lambda}, r_{\lambda}$, and $\gamma_{\lambda}$ only. This is due to the fact that these three variables uniquely determine the internal configuration of the triatomic system and hence the angle $\Delta_{\nu \lambda}$ between $R_{\nu}$ and $R_{\lambda}$ (see Fig. 1)。

Equations (A4), (A5), and (A7)-(A9) completely describe the $\lambda \rightarrow \nu$ transformation. It is useful to obtain the expressions they reduce to on the $\pi_{\nu \lambda}$ surface defined by Eq. (3.2a). In view of this definition and of Eq. (A6) we have, on this surface,

$$
\begin{aligned}
& r_{\nu}=r_{\lambda}, \\
& R_{\nu}=R_{\lambda} .
\end{aligned}
$$

From these and Eq. (A4) we get the very useful relation $R_{\lambda} / \gamma_{\lambda}=-\cot \alpha_{\nu \lambda} \cos \gamma_{\lambda}+\left(1+\cot ^{2} \alpha_{\nu \lambda} \cos ^{2} \gamma_{\lambda}\right)^{1 / 2}$

between $R_{\lambda} / r_{\lambda}$ and $\gamma_{\lambda}$ on this surface. Other important relations among the internal variables are obtained by substituting Eqs. (A10) and (A11) into (A7)。 We find

$$
\sin \gamma_{\nu}=\sin \gamma_{\lambda}
$$

and, after some algebraic effort,

$$
\cos \gamma_{\nu}=-\cos \gamma_{\lambda},
$$

which imply that

$$
\gamma_{\nu}=\pi-\gamma_{\lambda} \quad \bmod 2 \pi \text { 。 }
$$

Since $0 \leqslant \gamma_{\lambda} \leqslant \pi / 2$ or $3 \pi / 2 \leqslant \gamma_{\lambda}<2 \pi$, we see that $\pi / 2 \leqslant \gamma_{\nu}$ $\leqslant 3 \pi / 2$ on $\pi_{\nu \lambda}$.

From Eqs. (A10)-(A12) plus the expressions resulting from replacing those equations in (A9), the $\lambda \rightarrow \nu$ transformation equations on the $\pi_{\nu \lambda}$ matching surface are completely specified. In addition, since from Eqs.(A9) and (A10)

$$
\cos \Delta_{\nu \lambda}=\cos \alpha_{\nu \lambda}-\sin \alpha_{\nu \lambda} \cos \gamma_{\lambda}\left(r_{\lambda} / R_{\lambda}\right)
$$

and

$$
\sin \Delta_{\nu \lambda}=-\left(r_{\lambda} / R_{\lambda}\right) \sin \alpha_{\nu \lambda} \sin \gamma_{\lambda},
$$

and since from Eq. (A11), $r_{\lambda} / R_{\lambda}$ is a function of $\gamma_{\lambda}$ only (on $\pi_{\nu \lambda}$ ), so is $\Delta_{\nu \lambda}$.

We shall now show that Eq. (A11) when evaluated over the range $0 \leqslant \gamma_{\lambda} \leqslant \pi / 2$ and $3 \pi / 2 \leqslant \gamma_{\lambda}<2 \pi$ represents a half-plane whose edge is the $O Y_{\lambda}$ axis in the $O X_{\lambda} Y_{\lambda} Z_{\lambda}$ space defined in Sec. III. A and illustrated in Fig. 2 。 From Eqs. (3.1) and (A11), we obtain the simple expression $\cot \omega_{\lambda}=-\cot \alpha_{\nu \lambda} \cos \gamma_{\lambda} \quad 0 \leqslant \gamma_{\lambda} \leqslant \pi / 2$ and $3 \pi / 2 \leqslant \gamma_{\lambda}<2 \pi$

for the equation of $\pi_{\nu \lambda}$ in spherical polar coordinates In addition, from Eqs. (3.1b) and (A10) we have, on $\pi_{\nu \lambda}$,

$$
\omega_{\lambda}=\omega_{v} \text {. }
$$

To display the geometrical character of $\pi_{\nu \lambda}$ we switch from polar coordinates $\zeta, \omega_{\lambda}, \gamma_{\lambda}$ to Cartesian ones $X_{\lambda}, Y_{\lambda}, Z_{\lambda}$. Equation (A14) then becomes

$Z_{\lambda}=-\cot \alpha_{\nu \lambda} X_{\lambda} \quad 0 \leqslant \gamma_{\lambda} \leqslant \pi / 2$ and $3 \pi / 2 \leqslant \gamma_{\lambda}<2 \pi$ 。

This is the equation of a half-plane whose edge is the $Y_{\lambda}$ axis and which makes an angle $\pi-\alpha_{\nu \lambda}$ with the $O Z_{\lambda}$ axis (measured counter clockwise from $O Z_{\lambda}$ to $\pi_{\nu \lambda}$ as viewed from $O Y_{\lambda}$ )。

Another quantity of considerable importance in the matching procedure is the derivative operator $\partial / \partial n_{\nu \lambda}$ normal to the surface $\pi_{\nu \lambda}$ in the direction of increasing $\omega_{\lambda}$ (Sec. IV。A). Since this surface is a half-plane, this operator is easily found by using the standard expression

$$
\partial / \partial n_{\nu \lambda}=\hat{n}_{\nu \lambda} \cdot \nabla,
$$

where $\hat{n}_{\nu \lambda}$ is a unit vector normal to $\pi_{\nu \lambda}$ in the direction of increasing $\omega_{\lambda}$, and $\nabla$ is the gradient operator in $X_{\lambda} Y_{\lambda} Z_{\lambda}$ coordinates. Expressing Eq. (A17) in the spherical polar coordinates $\zeta, \omega_{\lambda}$, and $\gamma_{\lambda}$ we find

$$
\begin{aligned}
\frac{\partial}{\partial n_{\nu \lambda}}= & \frac{1}{\zeta} \frac{\sin \alpha_{\nu \lambda}}{\sin \omega_{\lambda}}\left[\left(\frac{\partial}{\partial \omega_{\lambda}}\right)_{\gamma_{\lambda}, \xi}+\cot \alpha_{\nu \lambda} \sin \gamma_{\lambda}\left(\frac{\partial}{\partial \gamma_{\lambda}}\right)_{\omega_{\lambda, \xi}}\right] \\
= & \frac{1}{\zeta}\left(\sin ^{2} \alpha_{\nu \lambda}+\cos ^{2} \alpha_{\nu \lambda} \cos ^{2} \gamma_{\lambda}\right)^{1 / 2}\left[\left(\frac{\partial}{\partial \omega_{\lambda}}\right)_{\gamma_{\lambda}, \xi}\right. \\
& \left.\quad+\cot \alpha_{\nu \lambda} \sin \gamma_{\lambda}\left(\frac{\partial}{\partial \gamma_{\lambda}}\right)_{\omega_{\lambda, \xi}}\right] \\
= & \frac{1}{\zeta} \frac{\sin \alpha_{\nu \lambda}}{\sin 2 \eta_{\lambda}}\left[\frac{1}{2}\left(\frac{\partial}{\partial \eta_{\lambda}}\right)_{\gamma_{\lambda, \xi}}+\cot \alpha_{\nu \lambda} \sin \gamma_{\lambda}\left(\frac{\partial}{\partial \gamma_{\lambda}}\right)_{\eta_{\lambda}, \xi}\right],
\end{aligned}
$$

and the indicated differentiations must be done on the full wavefunction with $\zeta, \omega_{\lambda}$ (or $\eta_{\lambda}$ ) and $\gamma_{\lambda}$ considered as independent variables before the relation between $\omega_{\lambda}$ (or $\eta_{\lambda}$ ) and $\gamma_{\lambda}$ describing $\pi_{\nu \lambda}$ is used. The third line of $\mathrm{Eq}$. (A18) results from the use of $\eta_{\lambda}$ rather than $\omega_{\lambda}$ as discussed in Sec. III. B。 Equation (A17) can also be evaluated in terms of $\nu$ arrangement channel coordinates, in which case we find

$$
\begin{aligned}
\frac{\partial}{\partial n_{\nu \lambda}}=-\frac{1}{\zeta} \frac{\sin \alpha_{\nu \lambda}}{\sin \omega_{\nu}}\left[\left(\frac{\partial}{\partial \omega_{\nu}}\right)_{\gamma_{\nu, \zeta}}-\cot \alpha_{\nu \lambda} \sin \gamma_{\lambda}\left(\frac{\partial}{\partial \gamma_{\nu}}\right)_{\omega_{\nu, \xi}}\right] \\
=-\frac{1}{\zeta_{\lambda}}\left(\sin ^{2} \alpha_{\nu \lambda}+\cos ^{2} \alpha_{\nu \lambda} \cos ^{2} \gamma_{\lambda}\right)^{1 / 2}\left[\left(\frac{\partial}{\partial \omega_{\nu}}\right)_{\gamma_{\nu, \xi}}\right. \\
\left.-\cot \alpha_{\nu \lambda} \sin \gamma_{\lambda}\left(\frac{\partial}{\partial \gamma_{\nu}}\right)_{\omega_{\nu, \xi}}\right] \\
=-\frac{1}{\zeta} \frac{\sin \alpha_{\nu \lambda}}{\sin 2 \eta_{\lambda}}\left[\frac{1}{2}\left(\frac{\partial}{\partial \eta_{\nu}}\right)_{\gamma_{\nu+\xi}}-\cot \alpha_{\nu \lambda} \sin \gamma_{\lambda}\left(\frac{\partial}{\partial \gamma_{\nu}}\right)_{\eta_{\nu, \xi}}\right] .
\end{aligned}
$$

\section{APPENDIX B: EIGENVALUES OF A MATRIX OF THE FORM $\mathbf{U}=\rho^{2} \mathbf{V}$}

In this Appendix we show that the potential matrices in the strong interaction and matching regions [Eqs. 
(3.48) and (3.61)] always have real eigenvalues even though they are not symmetric. These matrices have the general form

$$
U=\rho^{2} V \text {, }
$$

where the real matrix $\rho^{2}$ is the matrix representation of a positive definitive operator [Eq. $(3,49)]$ and therefore has positive real eigenvalues. $V$ is a real symmetric matrix whose eigenvalues may be positive, negative, or zero.

The first step in finding the eigenvalues of $\boldsymbol{U}$ involves a diagonalization of $\rho^{2}$,

$$
\overline{\mathrm{K}} \rho^{2} \mathrm{~K}=\Lambda \text {, }
$$

where $\Lambda$ is a diagonal matrix whose elements are the (positive) eigenvalues of $\rho^{2}$, and $\mathrm{K}$ is the real orthogonal matrix of eigenvectors of $\rho^{2}$. We now form the real symmetric matrix $\rho$ by

$$
\rho=\tilde{K} \Lambda^{1 / 2} K,
$$

where the diagonal matrix $\Lambda^{1 / 2}$ has diagonal elements which are the square roots of those of $\Lambda_{0} \rho$ behaves as if it were the "square root" of $\rho^{2}$ in many applications, since, from Eqs. (B2) and (B3),

$$
\rho \rho=\rho^{2} \text {. }
$$

Next, we define the real matrix $W$ as

$$
\mathbf{W}=\rho^{-1} \mathbf{U} \rho=\rho \mathbf{V} \rho,
$$

where the second equality in Eq. (B5) follows from $\mathrm{Eq}$. (B1). From Eq。(B5), it is obvious that $\boldsymbol{w}$ is real symmetric, and it may therefore be diagonalized by a real orthogonal matrix which we denote by $\mathbf{T}$ :

$$
\tilde{\mathbf{T}} \boldsymbol{W T}=\mathbf{E} .
$$

The diagonal matrix $\mathbf{E}$ contains the real eigenvalues of w.

Finally, if we define the nonorthogonal, nonsymmetric, but real matrix $S$ by

$$
S=\rho T,
$$

we obtain, from Eqs. (B5) and (B6),

$$
\mathbf{S}^{-1} \mathbf{U S}=\mathbf{E} \text {, }
$$

which proves that the matrix $\mathbf{S}$ diagonalizes $\mathbf{U}$ with the resulting real eigenvalues contained in $\mathbf{E}$.

\section{APPENDIX C: RELATIONS BETWEEN DISTINGUISHABLE ATOM SCATTERING AMPLITUDES FOR ATOM-DIATOM REACTIONS INVOLVING HOMONUCLEAR DIATOMIC MOLECULES}

In this Appendix we investigate the consequences of two-atom permutational symmetry (as discussed in Sec. VI. A) on the distinguishable-atom scattering amplitudes. We show that in collisions for which Eq. (6.1) is valid (i. e., collisions of an atom with a homonuclear diatomic molecule), the resultant scattering amplitudes $f_{\lambda v j}^{\nu v^{\prime} j^{\prime}}$ and $f_{\lambda v j}^{\kappa v^{\prime} j^{\prime}}$ are related by Eq. (6. 3) and that $f_{\lambda v j}^{\lambda v^{\prime} j^{\prime}}$ obeys Eq. (6.4). [The circumflex on $\hat{f}$ of Eq. (5.28) will be omitted in this Appendix. ]

We first rewrite the asymptotic behavior of the scattering solution Eq. (5.23) as

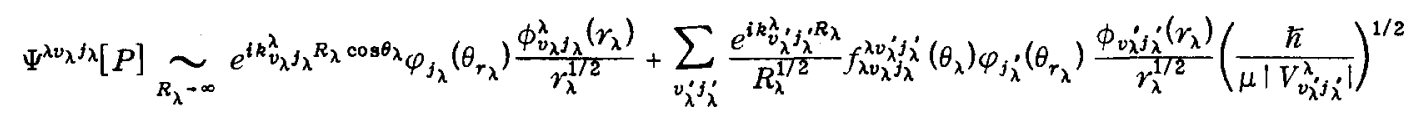

$$
\begin{aligned}
& \underset{R_{\nu} \rightarrow \infty}{\sim} \sum_{v_{\nu} j_{\nu}} \frac{e^{i k_{v_{\nu} j_{\nu}{ }^{\nu}}^{R_{\nu}}}}{R_{\nu}^{1 / 2}} f_{\lambda v_{\lambda} j_{\lambda} j_{\nu}}^{\nu v_{\nu}}\left(\theta_{\nu}\right) \varphi_{j_{\nu}}\left(\theta_{r_{\nu}}\right) \frac{\phi_{v_{\nu} j_{\nu}}^{\nu}\left(r_{\nu}\right)}{r_{\nu}^{1 / 2}}\left(\frac{\hbar}{\mu \mid V_{v_{\nu} j_{\nu}}^{\nu} 1}\right)^{1 / 2}
\end{aligned}
$$

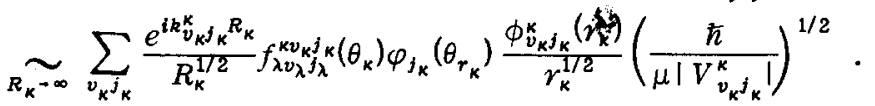

If the diatomic target in the incident channel is homonuclear, the physical system should be invariant to the operator $P_{\nu k}$ which interchanges the two identical atoms $A_{\nu}$ and $A_{k}$ involved. If we perform this interchange, the coordinates which define the system in each arrangement channel become (by inspection of Fig. 1)

$$
\left(\mathbf{R}_{\lambda}, \mathbf{r}_{\lambda}\right)-\left(\mathbf{R}_{\lambda},-\mathbf{r}_{\lambda}\right), \quad\left(\mathbf{R}_{\nu}, \mathbf{r}_{\nu}\right)-\left(\mathbf{R}_{\kappa},-\mathbf{r}_{\kappa}\right), \quad\left(\mathbf{R}_{\kappa}, \mathbf{r}_{\kappa}\right)-\left(\mathbf{R}_{\nu},-\mathbf{r}_{\nu}\right),
$$

which is equivalent to

$$
\begin{array}{lll}
\left(R_{\lambda}, \theta_{\lambda}\right)-\left(R_{\lambda}, \theta_{\lambda}\right), & \left(r_{\lambda}, \theta_{r_{\lambda}}\right)-\left(r_{\lambda}, \theta_{r_{\lambda}}+\pi\right), & \left(R_{\nu}, \theta_{\nu}\right)-\left(R_{k}, \theta_{k}\right), \\
\left(r_{\nu}, \theta_{r_{\nu}}\right) \rightarrow\left(r_{k}, \theta_{r_{k}}+\pi\right), & \left(R_{k}, \theta_{k}\right) \rightarrow\left(R_{\nu}, \theta_{\nu}\right), & \left(r_{k}, \theta_{r_{k}}\right)-\left(r_{\nu}, \theta_{r_{\nu}}+\pi\right) .
\end{array}
$$

If we make these substitutions into Eq. (C1) realizing that $\varphi_{j_{\lambda}}\left(\theta_{r_{\lambda}}+\pi\right)=(-1)^{\lambda_{\lambda} \varphi_{j_{\lambda}}}\left(\theta_{r_{\lambda}}\right)$, and appropriately relabel the quantum numbers which serve as summation indices, we obtain

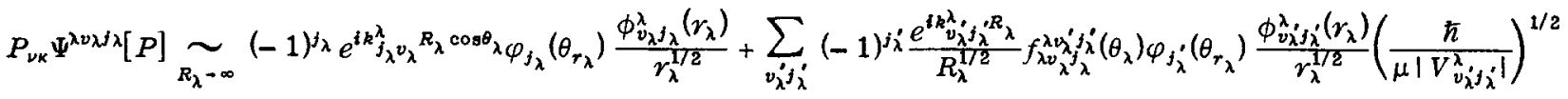

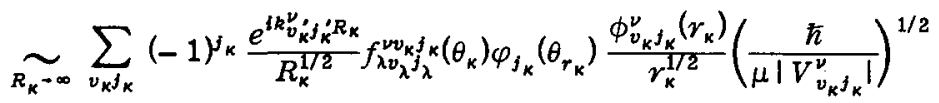

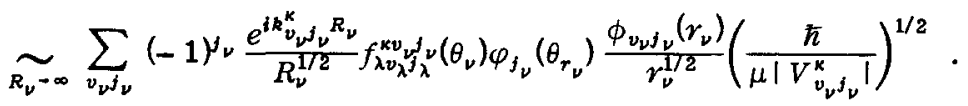


The line-wave part of (C3) differs only by a factor of $(-1)^{j_{\lambda}}$ from the corresponding part of (C1). Since the scattering solutions are unique (except for a normalization constant), this implies that this proportionality relation must hold over the entire configuration space, i. e., that

$$
P_{\nu \kappa} \psi^{\lambda v_{\lambda}{ }^{j}} \lambda[P]=(-1)^{j} \lambda \psi^{\lambda v} \lambda^{j} \lambda[P]
$$

everywhere. Replacement of Eqs. (C1) and (C3) into (C4) and identification of the corresponding outgoing wave parts in channel $\lambda$ immediately yields

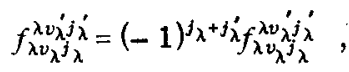

which is equivalent to Eq. (6.4). In order to compare the outgoing wave solutions in channels $\nu$ and $\kappa$, we first must realize that the $\phi_{v_{k^{j}} j_{k}}^{\nu}\left(r_{k}\right)$ (and $k_{v_{k} j_{k}}^{\nu}$ ) of Eq. (C3), and the $\phi_{v_{k} j_{k}}^{k}\left(r_{k}\right)$ (and $k_{v_{k} j_{k}}^{k}$ ) of Eq. (C1) are identical since these represent vibrational wavefunctions in the two product arrangement channels $A_{\kappa} A_{\lambda}$ and $A_{\lambda} A_{\nu}$, both of which are the same for a homonuclear target $A_{\nu} A_{\kappa}$. This allows us to compare the outgoing wave solutions in channels $\nu$ and $\kappa$ in Eq. (C4), obtaining

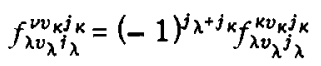

in both cases. Equation (C6) is identical to Eq. (6.3).

If atoms $A_{\lambda}$ and $A_{\nu}$ are identical, a derivation similar to that of (C4) leads to

$$
P_{\lambda \nu} \psi^{\lambda \nu \lambda^{j}} \lambda[P]=(-1)^{j} \psi^{\nu v_{\lambda} j_{\lambda}}[P],
$$

and if $A_{\lambda}$ and $A_{\mathrm{K}}$ are identical we get

$$
P_{\lambda k} \psi^{\lambda \nu_{\lambda}^{j} \lambda}[P]=(-1)^{j} \lambda \psi^{\kappa v} \lambda^{j} \lambda[P] \text {. }
$$

From Eq. (C7) results

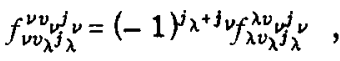

$$
\begin{aligned}
& f_{\nu v_{\lambda} j_{\lambda}}^{\lambda v_{\nu}^{j} j_{\nu}}=(-1)^{j_{\lambda}+j_{\nu}} f_{\lambda v_{\lambda}{ }^{j} v_{\lambda}}^{\nu v_{\nu}^{j} \nu},
\end{aligned}
$$

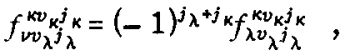

and analogous expressions stem from Eq. (C8):

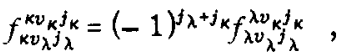

$$
\begin{aligned}
& f_{k v_{\lambda} j_{\lambda}}^{\lambda v_{k} j_{k}}=(-1)^{j_{\lambda}+j_{k}} f_{\lambda v_{\lambda} j_{\lambda}}^{k_{0} v_{k} j_{k}}, \\
& f_{k v_{\lambda} j_{\lambda}}^{v v_{\nu} j^{j}}=(-1)^{i_{\lambda}+f_{\nu}} f_{\lambda v_{\lambda} j_{\lambda}}^{\nu v_{\nu} j_{\nu}} \text {, }
\end{aligned}
$$

If all three atoms are identical, Eqs. (C4) - (C14) are all valid, as well as expressions resulting from cyclic permutations of indices $\lambda, \nu, \kappa$.

For reactions in three dimensions, Eqs. (C2) are still valid. The rotational diatomic wavefunctions $\varphi_{j_{\lambda}}\left(\theta_{r_{\lambda}}\right)$ are replaced by the spherical harmonics $Y_{j_{\lambda j_{j}} \lambda}\left(\theta_{r_{\lambda}}, \phi_{r_{\lambda}}\right)$, where $\theta_{r_{\lambda}}, \phi_{r_{\lambda}}$ are the spherical polar angles of $\mathbf{r}_{\lambda}$, and $j_{\lambda}, m_{j_{\lambda}}$ are the diatom's angular momentum quantum numbers. Replacement of $r_{\lambda}$ by $-r_{\lambda}$ is equivalent to replacing those polar angles by $\pi-\theta_{r_{\lambda}}$ and $\phi_{r_{\lambda}}+\pi$, respectively. The relation $Y_{j_{\lambda} m_{\lambda}}\left(\pi-\theta_{r_{\lambda}}, \phi_{r_{\lambda}}+\pi\right)=(-1)^{j_{\lambda}}$ $\times Y_{j_{\lambda} m_{\lambda}}\left(\theta_{r_{\lambda}}, \phi_{r_{\lambda}}\right)$, which is analogous to the relation $\varphi_{j_{\lambda}}\left(\theta_{r_{\lambda}}+\pi\right)=(-1)^{j_{\lambda}} \varphi_{j_{\lambda}}\left(\theta_{r_{\lambda}}\right)$ used to derive Eq. (C4). As a result, that equation, as well as Eqs. (C5)-(C14) are also valid in three dimensions, as long as the quantum numbers $v_{\lambda} j_{\lambda}$ (and their $\nu$ and $\kappa$ counterparts) are augmented by the diatomic rotational angular momentum projection quantum number $m_{j_{\lambda}}$.

\section{APPENDIX D: POSTANTISYMMETRIZATION FOR THE $\mathrm{H}+\mathrm{H}_{2}$ EXCHANGE REACTION}

Although correct expressions for the indistinguishable (antisymmetrized) cross sections in terms of the distinguishable atom reactive and nonreactive amplitudes have been listed by Doll, George, and Miller, ${ }^{44}$ who have obtained their results using the integrodifferential equation approach described by Miller, ${ }^{45}$ there seems to be confusion in the use of these expressions so we shall derive them here in order to clarify their meanings. We will follow the postantisymmetrization procedure outlined by Schiff ${ }^{23}$ which is somewhat more transparent than Miller's. In all of our treatment below we assume that the interaction potential is not spin dependent and that there are no external magnetic fields present.

We start by rewriting the asymptotic physical solution [Eq. (5.23)] for distinguishable-atom scattering (in the mass-scaled coordinate system). To simplify the notation we omit the symbol $[P]$ (which will be implied throughout this Appendix) and replace the labels $(\alpha, \beta, \gamma)$ by $(1,2,3)$. Therefore, $(\lambda \nu \kappa)$ will stand for one of the cyclic permutations $(1,2,3),(2,3,1)$, and $(3,1,2)$. Choosing the first of these we get

$$
\begin{aligned}
& \Psi^{1 v_{1} j_{1}}(1,2,3) \underset{R_{1} \rightarrow \infty}{\sim} e^{i \mathbf{k}_{v_{1} j_{1}}^{1} \cdot \boldsymbol{R}_{1}} W_{v_{1} j_{1}}^{1}(2,3)
\end{aligned}
$$

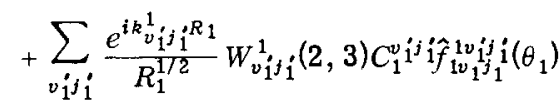

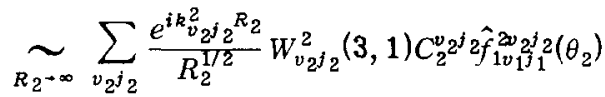

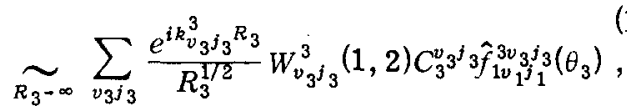

where

$$
\begin{aligned}
& W_{v_{\lambda} j_{\lambda}}^{\lambda}(\nu, \kappa)=\frac{\varphi_{j_{\lambda}}\left(\theta_{r_{\lambda}}\right) \phi_{\nu_{\lambda} j_{\lambda}}^{\lambda}\left(r_{\lambda}\right)}{r_{\lambda}^{1 / 2}} \\
& C_{\lambda}^{v_{\lambda} j_{\lambda}}=\left(\frac{\hbar}{\mu \mid V_{v_{\lambda} j_{\lambda}}^{\lambda} l}\right)^{1 / 2}
\end{aligned}
$$

Note that we have used the numbers 1,2 , and 3 as both an arrangement channel label and a particle label. Provided that we always permute particles in a cyclic way in our arrangement channel labeling, the two designations are identical and we will consider this to be the case here. This means that the coordinate $r_{1}$ is the internuclear vector from particle 2 to particle 3 (in the $R_{1}, r_{1}$ coordinate system), and therefore the 2, 3 diatomic molecule wavefunction is a function of $\mathrm{r}_{1}$. For $\mathrm{H}+\mathrm{H}_{2}$, the separated ar rangement channel quantum states are all identical, so the labels $v_{1} j_{1}, v_{2} j_{2}$, and $v_{3} j_{3}$ are essentially dummy indices and do not imply, for example, that $W_{v_{1} j_{1}}^{1}$ differs in its functional form from $W_{v_{2^{j}}{ }^{2}}^{2}$ or $W_{v_{3} j_{3}}^{3}$ when $j_{1}=j_{2}=j_{3}$ and $v_{1}=v_{2}=v_{3}$. As a result, we may drop the subscripts $\lambda$ of the quantum numbers $v_{\lambda} j_{\lambda}$ and the constants $C_{\lambda}$ as well as the superscript in the $W, k$, and $V$. Using Eq. (C4) for the cyclic permutation $(\lambda, \nu, \kappa)=(1,2,3)$, we get

$P_{23} \Psi^{1 v j}(1,3,2)=\Psi^{1 v j}(1,2,3)=(-1)^{j} \Psi^{1 v j}(1,3,2)$,

(D4a) 
TAELE 1. Antisymmetrized scattering amplitudes $\hat{f}\left(\begin{array}{l}\text { l) } \\ )_{v j}^{\prime \prime}\end{array} \mathrm{j}^{\prime \prime}\right.$ and their relation to the distinguishable atom amplitudes $\hat{f}_{1 v j^{1}}^{1 v^{\prime \prime}}$ and $\hat{f}_{2 v j^{\prime \prime}}^{1 v^{\prime \prime}} .^{\text {a }}$

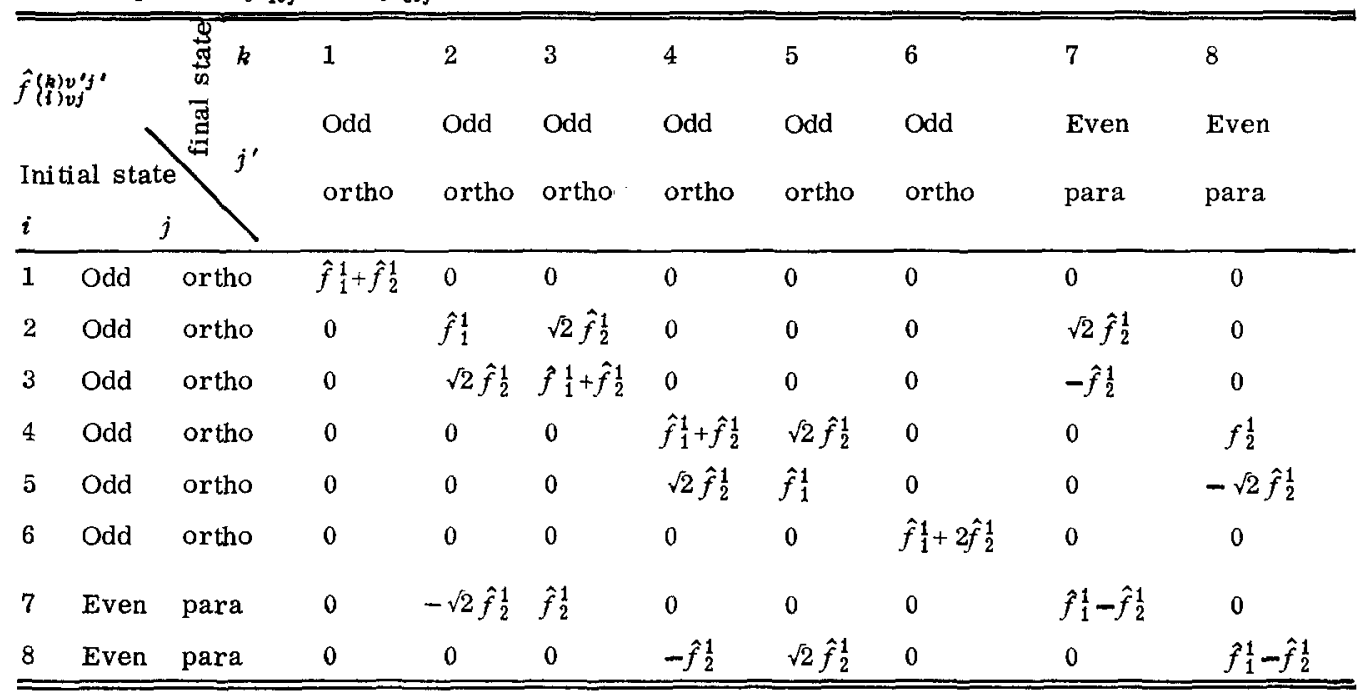

aThe indices $v j$ and $v^{\prime} j^{\prime}$ on the $\hat{f}_{1 v j}^{1 v j^{\prime \prime}}$ and $\hat{f}_{2 v j}^{1 v^{\prime \prime \prime}}$ are omitted.

which is valid in all of configuration space. In addition, using the cyclic permutation $(\lambda, \nu, k)=(2,3,1)$, Eq. (C7) furnishes

$$
P_{23} \Psi^{2 v j}(2,3,1)=(-1)^{j} \Psi^{3 v j}(3,1,2) .
$$

Let us now consider the spin wavefunctions of the separated atom plus diatom system in arrangement channel 1. Since the three hydrogen atoms have spin $\frac{1}{2}$, there are eight such wavefunctions which can be chosen to be

$$
\begin{aligned}
& v_{1}(1,2,3)=\alpha(1) \alpha(2) \alpha(3), \\
& v_{2}(1,2,3)=\beta(1) \alpha(2) \alpha(3), \\
& v_{3}(1,2,3)=\frac{1}{\sqrt{2}} \alpha(1)[\alpha(2) \beta(3)+\beta(2) \alpha(3)], \\
& v_{4}(1,2,3)=\frac{1}{\sqrt{2}} \beta(1)[\alpha(2) \beta(3)+\beta(2) \alpha(3)], \\
& v_{5}(1,2,3)=\alpha(1) \beta(2) \beta(3), \\
& v_{6}(1,2,3)=\beta(1) \beta(2) \beta(3), \\
& v_{7}(1,2,3)=\frac{1}{\sqrt{2}} \alpha(1)[\alpha(2) \beta(3)-\beta(2) \alpha(3)], \\
& v_{8}(1,2,3)=\frac{1}{\sqrt{2}} \beta(1)[\alpha(2) \beta(3)-\beta(2) \alpha(3)] .
\end{aligned}
$$

(D5h)

They are orthonormal eigenfunctions of $S_{x}$ but not of $S^{2}$ and have the symmetry property

$$
v_{i}(1,3,2)= \begin{cases}v_{i}(1,2,3) & i=1-6 \\ -v_{i}(1,2,3) & i=7,8 .\end{cases}
$$

The total separated atom plus diatom wavefunctions (including space and spin coordinates) are then

$$
\Phi^{1 v j(i)}(1,2,3)=\Psi^{1 v j}(1,2,3) v_{i}(1,2,3) \quad i=1-8
$$

where $(i)$ designates the spin state of the system. Since $\Phi^{1 v j}(1,2,3)$ must be antisymmetric with respect to permutation of particles 2 and 3 , we have the requirements [in view of Eqs. (D4) and (D6)] that

$$
j=\left\{\begin{array}{l}
\text { odd for } i=1-6 \text { (ortho states) } \\
\text { even for } i=7,8 \text { (para states) }
\end{array}\right.
$$

W/e now form the completely antisymmetric wavefunction $\Psi^{A v j(i)}(1,2,3)$ by taking the symmetric linear combination of the partly antisymmetric wavefunctions $\Phi^{\lambda v j}(\lambda \nu \kappa)$ for the three cyclic permutations of $(1,2,3)$ having the same initial $v j$ :

$\Psi^{A v j(i)}=\Phi^{1 v j(i)}(1,2,3)+\Phi^{2 v j(i)}(2,3,1)+\Phi^{3 v j(i)}(3,1,2)$

$$
i=1-8 \text {. (D9) }
$$

Equations (D4), (D6), and (D8) can be used to prove that $\Psi^{A \nu f(i)}$ is antisymmetric under a permutation of any two of the three particles in the system. This then is the correct scattering solution, and its asymptotic behavior can be used to determine the correct antisymmetrized scattering amplitudes. This asymptotic behavior can be determined by evaluating $\Psi^{A v j(i)}$ at large values of $R_{1}$ (or $R_{2}$ or $R_{3}$ ), which yields

$$
\begin{aligned}
\Psi^{A v j(i)}(1,2,3) \underset{R_{1} \rightarrow \infty}{\sim} & e^{i \mathbf{k}_{v j} \cdot \mathbf{R}_{1}} W_{v j}(2,3) v_{i}(1,2,3) \\
& +\sum_{v^{\prime} j} \frac{e^{i k_{v}^{\prime} \cdot R_{1}}}{R_{1}^{1 / 2}} W_{v^{\prime} j}^{1},(2,3) C^{v^{\prime} j^{\prime}}\left(\hat{f}_{1 v j}^{1 v^{\prime} j^{\prime}} v_{i}(1,2,3)+\hat{f}_{2 v j}^{1 v^{\prime \prime}{ }^{\prime}} v_{i}(2,3,1)+\hat{f}_{3 v j}^{1 v^{\prime} j^{\prime}} v_{i}(3,1,2)\right) .
\end{aligned}
$$


This has the form of a physical scattering solution, but the expression in large parentheses must be re-expressed in terms of the $v_{k}(1,2,3)$ spin functions of the final separated atom-diatom $(k=1-8)$. Accordingly, we write

$$
\begin{aligned}
& \hat{f}_{1 v j}^{1 v^{\prime} j^{\prime}} v_{i}(1,2,3)+\hat{f}_{2 v j}^{1 v^{\prime} j^{\prime}} v_{i}(2,3,1)+\hat{f}_{3 v j}^{1 v^{\prime} j^{\prime}} v_{i}(3,1,2) \\
& =\sum_{k=1}^{8} f_{(i) v^{\prime} j}^{A(k) v^{\prime} j^{\prime}} v_{k}(1,2,3)
\end{aligned}
$$

where $f_{(i) v j}^{A(k) v^{\prime} j{ }^{\prime}}$ is the antisymmetrized scattering amplitude for scattering from initial state $(i) v j$ (spin state $i$ ) to final state $(k) v^{\prime} j^{\prime}$ [where Eq. (D8) must be satisfied for both of these states]. We can solve for the $f_{(i) v j}^{A(k) v^{\prime} j}$ ' by multiplying Eq. (D11) by $v_{l}(1,2,3)$, integ rating over all spin variables and then replacing the index $l$ by $k$ in the result. The resulting expressions for $f_{(i) v_{1} j_{1}^{(k)} j^{j}{ }_{i}}$ in terms of the distinguishable-atom scattering amplitudes are given in Table $\mathrm{I}$. The expressions in that table have been simplified by the use of the relation [see Eqs. (6.3)]:

$$
\hat{f}_{3 v j}^{1 v^{\prime} j^{\prime}}=(-1)^{j+j^{\prime}} f_{3 v j}^{1 v^{\prime} j^{\prime}}
$$

In the notation of Doll, George, and Miller, ${ }^{44} \hat{f}_{1 v j}^{1 v^{\prime} f^{\prime}}$ is the direct while $\hat{f}_{2 v j}^{l v^{\prime} j^{\prime}}$ is the exchange scattering amplitude.

The state-to-state cross sections are [from Eq. (5.24)]

$$
\sigma_{(i) v j}^{(j) v^{\prime} j}=\frac{1}{k_{v j}}\left|\hat{f}_{(i) v j}^{(j) v^{\prime} j^{\prime}}\right|^{2}
$$

and the cross sections of Eq. (6.5) are obtained by summing Eq. (D13) over final spin states and averaging over initial ones. As an example, the para-to-ortho cross section [Eq. $(6.5 \mathrm{~b})]$ is given by (dropping the $v j, v^{\prime} j^{\prime}$ indices but retaining the spin labels)

$$
\begin{aligned}
\sigma_{\mathrm{p}}^{0} & =\frac{1}{2}\left(\sigma_{7}^{1}+\sigma_{7}^{2}+\sigma_{7}^{3}+\sigma_{7}^{4}+\sigma_{7}^{5}+\sigma_{7}^{6}+\sigma_{8}^{1}+\sigma_{8}^{2}+\sigma_{8}^{3}+\sigma_{8}^{4}+\sigma_{8}^{5}+\sigma_{8}^{6}\right) \\
& =\frac{3}{k}\left|\hat{f}_{2}^{1}\right|^{2} .
\end{aligned}
$$

The derivation of the antisymmetrized scattering amplitudes of Table I was based on the validity of Eqs. (D4) and (D6). The latter comes from the properties of the spin states of Eq. (D5) which are the correct ones for the three-dimensional world. Equations (D4a) and (D4b) came from Eqs. (C4) and (C7), respectively, which as pointed out at the end of Appendix $\mathrm{C}$, are also valid in three dimensions after including the projection quantum numbers $m_{j}$. The other characteristics of Eq. (D1) which change in going from $2 \mathrm{D}$ to $3 \mathrm{D}$ are irrelevant for the derivation of the antisymmetrized scattering amplitudes. As a result, the formulas of Table I are also valid in three dimensions when augmented by the quantum numbers $m_{j}$. A similar statement is valid for Eqs. (6.5) if $\overrightarrow{k_{v j}^{\lambda}}$ is replaced by $\left(\bar{k}_{v j}^{\lambda}\right)^{2}$.

*Work supported in part by the United States Air Force Office of Scientific Research (Grant No. AFOSR-73-2539).

†Work performed in partial fulfillment of the requirements for the Ph.D. degree in Chemistry at the California Institute of Technology. Present address: Department of Chemistry, Northwestern University, Evanston, Illinois 60201.

¥Present address: Soreq Nuclear Center, Yavne, Israel; and
Department of Chemical Physics, The Weizmann Institute of Science, P. O. Box 26, Rehovot, Israel.

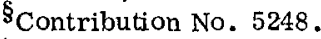

${ }^{1}$ E. M. Mortenson and K. S. Pitzer, Chem. Soc。(London) Spec. Publ. 16, 57 (1962); E. M. Mortenson, J. Chem. Phys. 48, 4029 (1968).

${ }^{2}$ D. J. Diestler and V. McKoy, J. Chem. Phys. 48, 2951 (1968).

${ }^{3}$ C. C. Rankin and J. C. Light, J. Chem. Phys. 51, 1701 (1971); G. Miller and J. C. Light, J。Chem. Phys. 54, 1635 (1971); ibid. 54, 1643 (1971).

${ }^{4}$ D. J. Truhlar and A. Kuppermann, J. Chem. Phys. 52, 3841 (1970); ibid. 56, 2232 (1972).

${ }^{5}$ A. Kuppermann, Proc. Conf. Potential Energy Surf. Chem. U. C. Santa Cruz, August 1970 (1971), p. 121; Electronic and Atomic Collisions, Proceedings of VII ICPEAC (NorthHolland, Amsterdam, 1971), p. 3.

${ }^{6}$ D. J. Diestler, J. Chem. Phys. 54, 4547 (1971).

${ }^{7}$ B. R. Johnson, Chem. Phys. Lett. 13, 172 (1972).

${ }^{8}$ S. F. Wu and R. D. Levine, Mol. Phys. 22, 881 (1971).

${ }^{9}$ E. Shipsey, J. Chem. Phys. 58, 232 (1973).

${ }^{10} \mathrm{G}$. C. Schatz, J. Bowman, and A. Kuppermann, J. Chem. Phys. 58, 4023 (1973); G. C. Schatz and A. Kuppermann, J. Chem. Phys. 59, $964(1973)$; J. M. Bowman, G. C. Schatz, and A. Kuppermann, Chem. Phys. Lett. 24, 378 (1974); G. C. Schatz, J. M. Bowman, and A. Kuppermann, J. Chem. Phys. 63, 674, 885 (1975).

${ }^{11} \mathrm{~J}$. T. Adams, R. L. Smith, and E. F. Hayes, J. Chem. Phys, 61, 2193 (1974).

${ }^{12} \mathrm{M}$. Baer and D. J. Kouri, Chem. Phys. Lett. 24, 37 (1974); M. Baer, J. Chem. Phys, 60, 1057 (1974); A. Persky and M. Baer, J. Chem. Phys. 60, 133 (1974); M. Baer, U. Hal avee, and A. Persky, J. Chem. Phys. 61, 5122 (1974).

${ }^{13}$ P. B. Middleton and R. E. Wyatt, J. Chem. Phys. 56, 2702 (1972); E. A. MeCollough and R. E. Wyatt, J. Chem. Phys. $54,3578,3592(1971)$.

${ }^{14}$ R. P. Saxon and J. C. Light, J. Chem. Phys. 56, 3874, 3885 (1972).

${ }^{15} \mathrm{~A}$. Altenberger-Siczek and J. C. Light, J. Chem. Phys. 61, 4373 (1974).

${ }^{16} \mathrm{G}$. Wolken and M. Karplus, J. Chem. Phys. 60, 351 (1974).

${ }^{17}$ (a) A. B. Elkowitz and R。E. Wyatt, J. Chem. Phys.62, 2504, 3683 (1975); (b) S. A. Harms and R. E. Wyatt, J. Chem. Phys. 57, 2722 (1972); ibid. 62, 3162, 3173 (1975).

${ }^{18} \mathrm{M}$. Baer and D. J. Kouri, Chem. Phys. Lett. 11, 238 (1971); J. Chem. Phys. 56, 1758 (1972); ibid. 57, 3991 (1972).

${ }^{19}$ A. Kuppermann, G. C. Schatz, and M. Baer, J. Chem. Phys. 61,4362 (1974).

${ }^{20}$ G. C. Schatz and A. Kuppermann, J. Chem. Phys. 65, 4624 (1976), following paper.

${ }^{21}$ For reactions for which one of the three arrangement channels is energetically inaccessible, the integration needs to be done usually in only the two remaining open arrangement channels and a somewhat different and simpler matching procedure is required. This situation is not described in this paper although it can be handled by a straightforward modification of the theory here presented.

${ }^{22}$ A. Kuppermann and G. C. Schatz, J. Chem. Phys. 62, 2502 (1975); see also the second (p. 4642) and third (p. 4668) paper following this one.

${ }^{23}$ L. I. Schiff, Quantum Mechanics (McGraw-Hill, New York, 1968), 3rd ed., p. 384-395.

${ }^{24}$ A. M. Lane and R. G. Thomas, Rev. Mod. Phys. 30, 257 (1958); these authors use the term "collision matrix" in lieu of "scattering matrix."

${ }^{25}$ L. M. Delves, Nucl. Phys. 9, 391 (1959); 20, 275 (1960). ${ }^{26} \mathrm{D}$. Jepsen and J. O. Hirschfelder, Proc. Natl. Acad. Sci. 45,249 (1959).

${ }^{27}$ F. T. Smith, J. Math. Phys. 3, 735 (1962). Different sets of mass-scaled coordinates have been used by R. A. Marcus, J. Chem. Phys. 41, 603 (1964), by K. T. Tang, B. Kleinman, and M. Karplus, J. Chem. Phys. 50, 1119 (1969), and by 
many others, but they do not display all of the convenient properties of the coordinates of Eq. (2.2).

${ }^{28}$ This is true because the incident plane-(i.e., line-) wave solution discussed in Sec. V.B contains a whole spectrum of total angular momenta (corresponding classically to a range of impact parameters), many of which contribute to the reaction.

${ }^{29}$ The angles $\theta_{\nu}$ and $\theta_{\lambda}$ differ by an angle $\Delta_{\nu \lambda}$ which is a function of $r_{\lambda}, R_{\lambda}$, and $\gamma_{\lambda}$ only as seen in Eqs. (A8) and (A9). In addition, Eqs. (A7) show that $\gamma_{\nu}$ also depends on those three variables only. Therefore, $\varphi_{J}\left(\theta_{\lambda}\right)$ is also an eigenfunction of $\hbar / i\left(\partial / \partial \theta_{\nu}\right)_{\gamma_{\nu}}$ with the same eigenvalue $J \hbar$. The quantum number $J$ is, as a result, independent of the arrangement channel $\lambda$ used to obtain it.

${ }^{30}$ (a) A. Kuppermann, Chem. Phys. Lett. 32, 374 (1975); (b) ibid. J. Chem. Phys. (to be published).

${ }^{31}$ R. N. Porter and M. Karplus, J. Chem. Phys. 40, 1105 (1964).

${ }^{32}$ Actually, values of $\omega_{\lambda_{0}}$ smaller than $\pi-\alpha_{\nu \lambda}$ and of $\omega_{\lambda_{1}}$ greater than $\pi / 2$ are also permissible but are not needed to determine the wavefunction on the matching half-plane $\pi_{\nu \lambda}$.

${ }^{33}$ Handbook of Mathematical Functions, edited by M. Abramowitz and I. A. Stegun (National Bureau of Standards, Washington, D.C., 1964), Chap. 9.

${ }^{34} \mathrm{D}$. G. Truhlar, dissertation, California Institute of Technology, 1970, Appendix 4.

${ }^{35}$ R. Gordon, J. Chem. Phys. 51, 14 (1969).

${ }^{36}$ We used a version of Gordon's method developed by A. Wagner for inelastic atom-diatom collisions. See A. Wagner and V. Mckoy, J. Chem. Phys. 58, 5561 (1973).

${ }^{37}$ To clarify this concept, let us consider a scattering solution consisting of an incoming wave in arrangement channel $\lambda$ and outgoing waves in all three arrangement channels, $\lambda, \nu, \kappa$.
There will, in general, be $N$ of these kinds of scattering solutions which are linearly independent (for a coupled-channel expansion truncated at $N$ terms in each arrangement channel), along with $N$ having incoming waves in channel $\nu$ and $N$ with incoming waves in channel $\kappa$ for a total of $3 N$ solutions. The latter two sets of solutions will have only outgoing waves in channel $\lambda$ and, in the absence of the inclusion of dissociated states, it will be impossible to linearly combine these latter $2 N$ solutions to generate the $N$ sets of solutions which have incoming waves in channel $\lambda$. Therefore we have $3 N$ scattering solutions which are linearly independentwhen the entire configuration space is considered, although only $2 N$ of them are independent in any one asymptotic arrangement channel.

${ }^{38}$ N. S. F. Mott and H. S. W. Massey, The Theory of Atomic Collisions (Clarendon, Oxford, 1956), 3rd ed., Chaps, 14 and 15.

${ }^{39}$ Note that there are a few differences in phases and signs between the formulas which describe coplanar scattering and those for 3D. ${ }^{38}$ These changes result from the differences between the phases of the cylindrical and spherical Bessel functions ${ }^{33}$ and, of course, have no physical significance.

${ }^{40}$ This expression is obtained from Ref. 33, p. 351, Eq. 9.1.41 by setting $t=i \exp \left(i \theta_{\lambda} \cdot\right)$.

${ }^{41}$ R. B. Walker and R. E. Wyatt, J. Chem. Phys. 61, 4839 (1974).

${ }^{42} \mathrm{~J}$. R. Taylor, Scattering Theory (Wiley, New York, 1972), Chap. 22.

${ }^{43}$ D. G. Truhlar and J. Abdallah, Phys. Rev. A 9, 297 (1974).

${ }^{44}$ J. D. Doll, T. F. George, and W. H. Miller, J. Chem. Phys. 58, 1343 (1973).

${ }^{45}$ W. H. Miller, J. Chem. Phys, 50, 407 (1969). 